\title{
Multifrequency Behaviour of High Energy Cosmic Sources in the GW Era
}

\section{Franco Giovannelli*}

INAF - Istituto di Astrofisica e Planetologia Spaziali, Via del Fosso del Cavaliere, 100, 00133

Roma, Italy

E-mail: franco.giovannellieinaf.it

\section{Lola Sabau-Graziati}

INTA-Dpt. Cargas Utiles y Ciencias del Espacio, C/ra de Ajalvir, Km 4 - E28850 Torrejón de Ardoz, Madrid, Spain

E-mail: sabaumdeinta.es

We will discuss the importance of the "Multifrequency Astrophysics" as a pillar of an interdisciplinary approach for the knowledge of the physics of our Universe. Indeed, as largely demonstrated in the last decades, only with the multifrequency observations of cosmic sources it is possible to get near the whole behaviour of a source and then to approach the physics governing the phenomena that originate such a behaviour. In spite of this, a multidisciplinary approach in the study of each kind of phenomenon occurring in each kind of cosmic source is even more powerful than a simple "astrophysical approach". A clear example of a multidisciplinary approach is that of "The Bridge between the Big Bang and Biology". This bridge can be described by using the competences of astrophysicists, planetary physicists, atmospheric physicists, geophysicists, volcanologists, biophysicists, biochemists, and astrobiophysicists. The unification of such competences can provide the intellectual framework that will better enable an understanding of the physics governing the formation and structure of cosmic objects, apparently uncorrelated with one another, that on the contrary constitute the steps necessary for the life (e.g. Giovannelli, 2001). Indeed, a lot of the future research in astrophysics will be focussed on the discovery of exoplanets and on the possibility to detect signals for alien life somewhere in the Galaxy. An extension to a multidisciplinary approach is coming from the use of historical news reported in "old chronicle" that are a fundamental source for the newborn "archaeoastronomy".

There are many problems in performing Simultaneous Multifrequency, Multisite, Multiinstrument, Multiplatform Measurements due to: (i) objective technological difficulties; (ii) sharing common scientific objectives; (iii) problems of scheduling and budgets; (iv) politic management of science. All these kind of measurements converge in what is now called Multimessenger Astrophysics, after the detection of gravitational wave events (GWEs) and the search for the electromagnetic counterparts of such events.

A part of this paper is a summary of an updated version of the book "The Impact of the Space Experiments on Our Knowledge of the Physics of the Universe" published in 2004 by the Kluwer Academic Publishers, reprinted from the review paper by Giovannelli, F. \& Sabau-Graziati, L.: 2004, Space Sci. Rev. 112, 1-443 (GSG2004), and subsequent considered lucubrations.

In this paper we will provide several example that marked the continuous evolution on the knowledge of the physics of our Universe.

Multifrequency Behaviour of High Energy Cosmic Sources - XIII - MULTIF2019

3-8 June 2019

Palermo, Italy

${ }^{*}$ Speaker. 


\section{Introduction}

A part of this paper is a summary of an updated version of the book "The Impact of the Space Experiments on Our Knowledge of the Physics of the Universe" published in 2004 by the Kluwer Academic Publishers, reprinted from the review paper by Giovannelli, F. \& Sabau-Graziati, L.: 2004, Space Sci. Rev. 112, 1-443 (GSG2004), and subsequent considered lucubrations. However we will discuss about the pillars that take the Bridge Between the Big Bang and Biology (Giovannelli, 2001). This bridge undoubtedly exists because we are present here regardless of the origin of our Universe. So we must understand how to cross this bridge and understand what are the tools that allow us to make out the structure of the pillars supporting the bridge.

In order to cross this bridge, as always when we cross a bridge, we must advance slowly, step by step, with continuity, because everything is smoothly linked in the magma of the Universe: from the infinitely small to infinitely big, a shown in Fig. 1 (Rees, 1988).

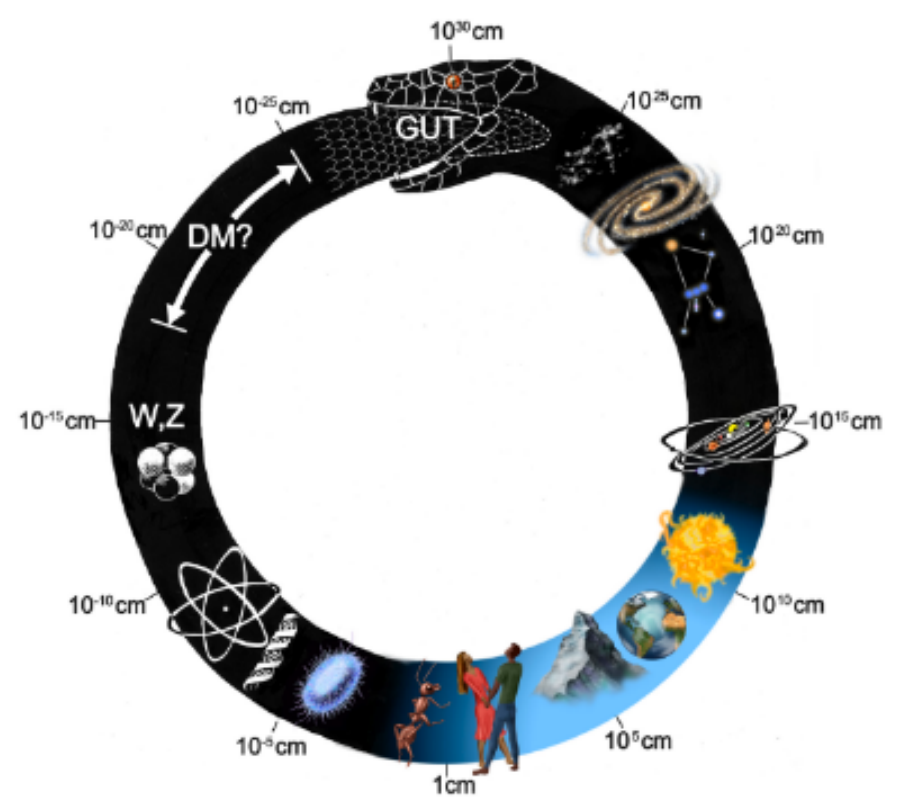

Figure 1: From the infinitely small to infinitely big (adopted by Rees, 1988).

In nature, nothing is isolated. Everything is related to the surrounding environment in a more or less strong way. However, the link exists. Fig 2 (upper left) shows a section of the metabolic network of a "simple" bacterium. Note that each point (each chemical compound) is connected to any other point through the complexity of the network (Luisi \& Capra, 2014). Fig. 2 (Upper right) shows the cosmic network: each point is connected to any other point through the complexity of the network (Credit: Andrew Pontzen/Fabio Governato, 2014; see also in (https://it.wikipedia.org/wiki/Cosmologia del plasma). The large-scale structure of the Universe, as traced by the distribution of galaxies, is now being revealed by large-volume cosmological surveys. The structure is characterized by galaxies distributed along filaments, the filaments connecting in turn to form a percolating network. The objective of Shandarin, Habib \& Heitmann (2010) was to quantitatively specify the underlying mechanisms that drive the formation of the cosmic network. 
By combining percolation-based analyzes with N-body simulations of gravitational structure formation, they elucidate how the network has its origin in the properties of the initial density field (nature) and how its contrast is then amplified by the nonlinear mapping induced by the gravitational instability (nurture). Fig. 2 (Lower left) shows the human body network: each point (organ) is connected to any other point (organ) through the complexity of the network (Luisi \& Capra, 2014). Fig. 2 (Lower right) shows the human society network: each point is connected to any other point through the complexity of the network (Luisi \& Capra, 2014).
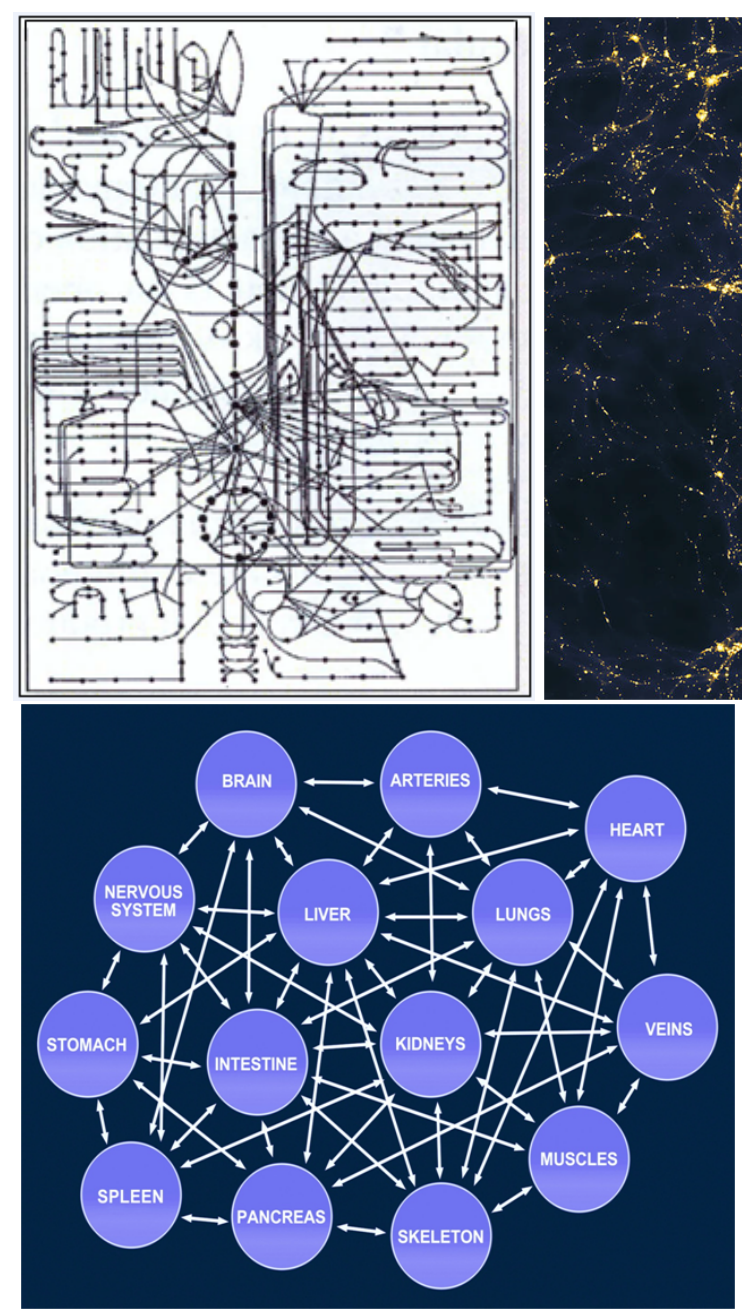
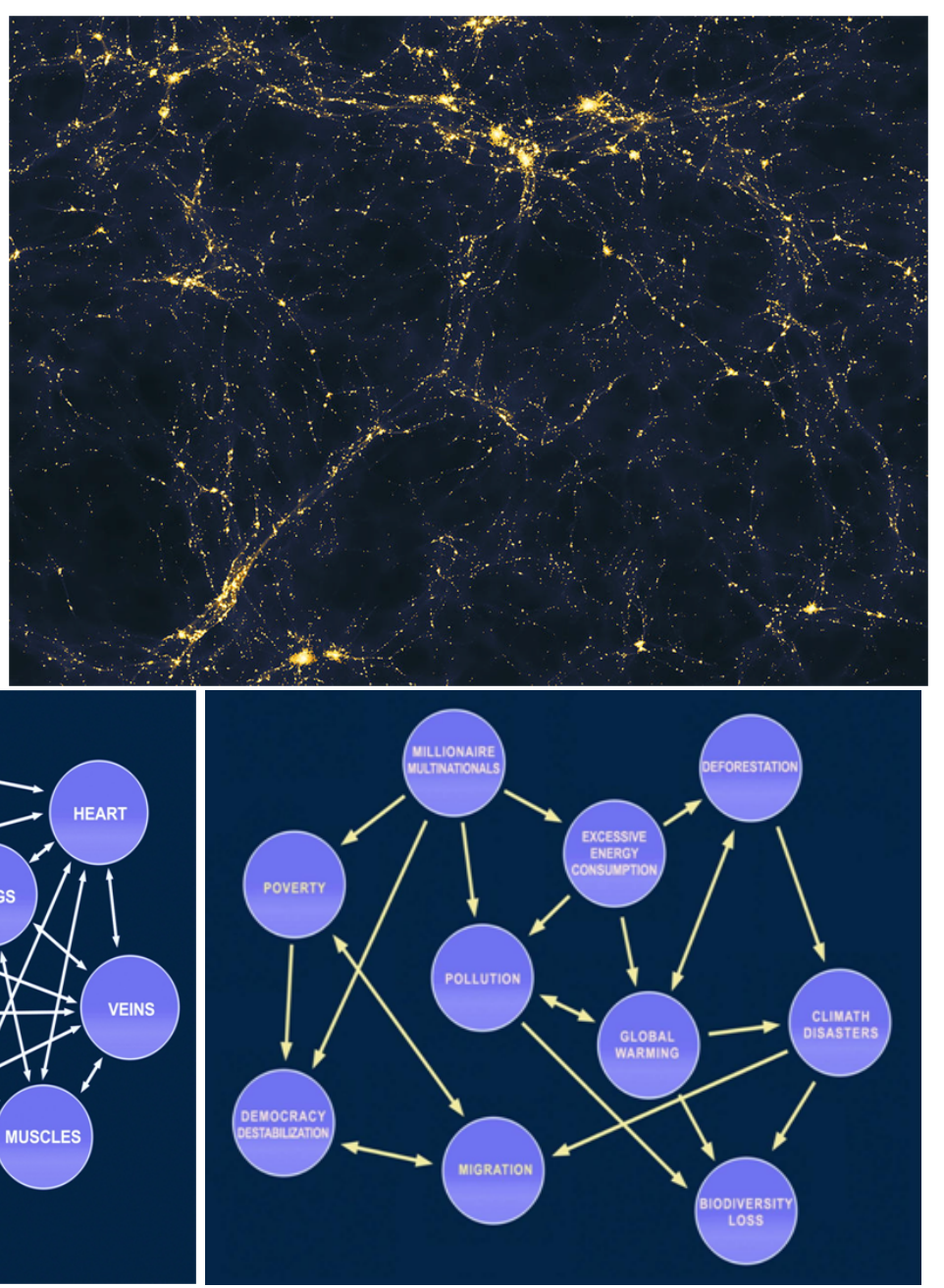

Figure 2: Upper left panel: Section of the metabolic network of a "simple" bacterium (Luisi \& Capra, 2014). Upper right panel: the "cosmic network" (Credit: Andrew Pontzen/Fabio Governato, 2014) see also in (https://it.wikipedia.org/wiki/Cosmologia del plasma). Lower left panel: the human body network. Lower right panel: the human society network (Luisi \& Capra, 2014).

Figure 3 shows a sample of human population. All these people follow the cycle: birth, growth, aging, death. This is a general rule of the nature. Indeed also all the components of the Universe follow the same cycle: birth, growth, aging, death, as the living beings. Therefore for a complete understanding of the history of the Universe it is necessary to search along that cycle.

Therefore a natural question arises: how is it possible to explore the bridge between the big 
bang and biology?

Albert Einstein gave a fundamental input for this purpose: We can't solve problems by using the same kind of thinking we used when we created them. And we can add a simple sentence: We can attach each kind of problem in a way as general as possible, and in any case it is necessary to go on without blinkers.
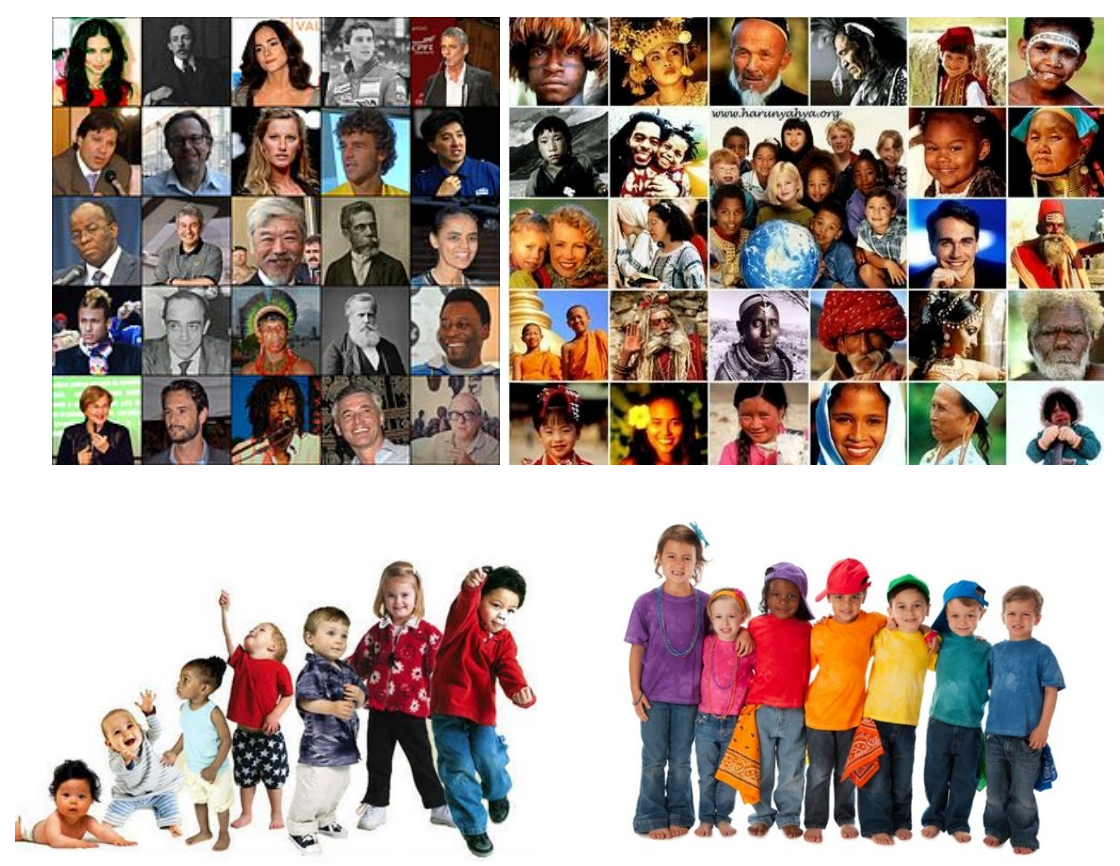

Figure 3: Different samples of human population. All of them follow the cycle: birth, growth, aging, death.

\subsection{Accretion Processes in Cosmic Sources}

Accretion is a universal phenomenon that takes place in the vast majority of astrophysical objects. The progress of ground-based and space-borne observational facilities has resulted in the great amount of information on various accreting astrophysical objects, collected within the last decades. The accretion is accompanied by the process of extensive energy release that takes place on the surface of an accreting object and in various gaseous envelopes, accretion disk, jets and other elements of the flow pattern. The results of observations inspired the intensive development of accretion theory, which, in turn, enabled us to study unique properties of accreting objects and physical conditions in the surrounding environment. One of the most interesting outcomes of this intensive study is the fact that accretion processes are, in a sense, self-similar on various spatial scales from planetary systems to galaxies.

This fact gives us new opportunities to investigate objects that, by various reasons, are not available for direct study.

Cataclysmic variable stars are unique natural laboratories where one can conduct the detailed observational study of accretion processes and accretion disks. Indeed, among the cosmic systems where accretion processes occur, undoubtedly, non-magnetic CVs, intermediate polars and polars constitute the most powerful probe to test our theories of the various modes of accretion. The 


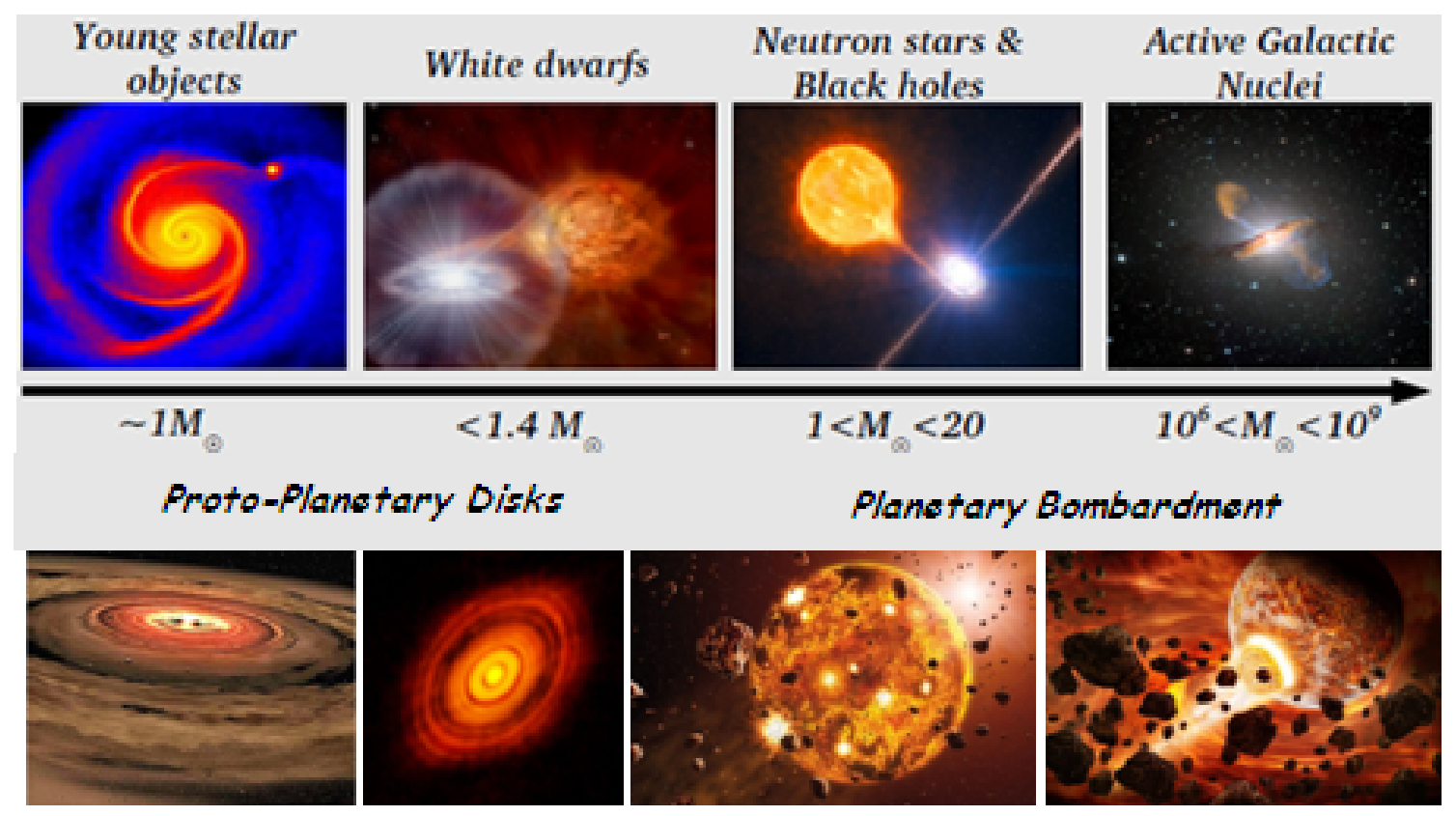

Figure 4: Accretion processes in different cosmic sources (adopted from Giovannelli \& Sabau-Graziati, 2016, after Scaringi, 2015).

reason is rather simple: CVs are enough close to us and their processes develop in time-scales relatively easy to be followed and enough energetic to be easily detected. The long term evolution of CV systems accreting at a prohibitive rate has become a hot topic both in terms of the fate of such systems (all sorts of supernovae) and the microphysics of Eddington and super Eddington mass accretion and mass loss flows. In particular we stress one of the hottest topics in present day astrophysics, namely the progenitors of SN-Ia. This problem is connected with fundamental issues in cosmology. Novae and recurrent novae are the most promising progenitor candidates but so far could not be nailed down.

Figure 4 shows a sketch of cosmic systems where accretion processes occur (Giovannelli \& Sabau-Graziati, 2016a, after Scaringi, 2015).

A series of international workshops on Accretion Processes in Cosmic Sources: Young Stellar Objects, Cataclysmic Variables (CVs) and Related Objects, X-ray Binary Systems, Active Galactic Nuclei was organized by us in collaboration with several colleagues of different international institutions, and took place in Saint Petersburg (Russian Federation) on September 2016, and September 2018. The proceedings discuss in details the physics of accretion processes in all the cosmic sources shown in Fig. 4 (Giovannelli \& Sabau-Graziati, 2016b, 2018a).

\subsection{About Cataclysmic Variables}

Historically, the classification of CVs was based on the optical outburst properties, by which one may distinguish four groups: (i) classical novae; (ii) recurrent novae; (iii) dwarf novae; (iv) nova-like objects.

This classification, however, is neither self-consistent nor adequate and it is much better to consider primarily the observed accretion behaviour (Smak, 1985). 
Figure 5 (left panel) shows the main characteristics of the visual light curves of classical novae (top panel) and of dwarf novae of the U Gem, Z Cam, and SU UMa types (lower three panels) (Ritter, 1992). In the right panel of Fig. 5 different kind of humans are reported. They show similarities with the light curves of CVs. Apparently they are different, but all of them belong to the same Human Species, like all the CVs are CVs.
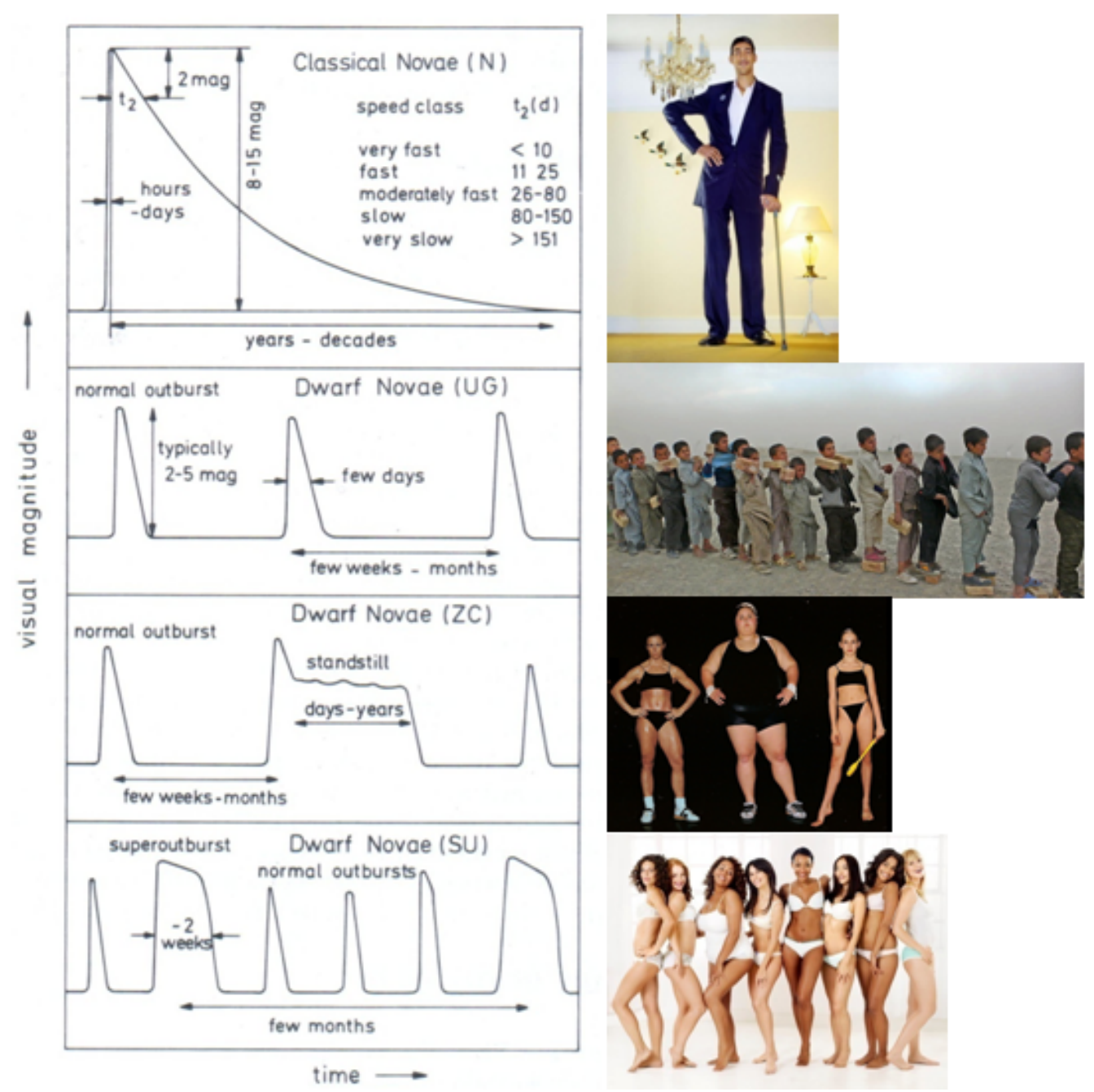

Figure 5: Left panel: the main characteristics of the visual light curves of CVs (Ritter, 1992). Right panel: different characteristics of humans.

Therefore, it is necessary to find a method as general as possible to describe the behavior of CVs. This can be obtained looking at the Accretion Behaviour and Magnetic Field.

\subsection{Classification on the base of the magnetic field intensity}

The accretion structure depends on the magnetic field of white dwarf (B) and on the transfer mass rate.

Following the popular classification, depending on B it is possible to classify CVs in three groups: 
- Non Magnetic CVs (NMCVs): B $\sim 10^{4}-10^{6} \mathrm{G}$;

- Intermediate Polars (IPs): $\mathrm{B} \sim 10^{6}-10^{7} \mathrm{G}$;

- Polars (MCVs): B $\sim 10^{7}-10^{8} \mathrm{G}$.

Figure 6 (upper panel) shows a sketch of such a classification, and the lower panel shows from the left to the right the 3D MHD simulations of the so-called non-magnetic CVs (NMCVs) (B $\left.\sim 10^{4}-10^{5} \mathrm{G}\right)$, intermediate polars (IPs) $\left(\mathrm{B} \sim 10^{6}-10^{7} \mathrm{G}\right)$, and polars $(\mathrm{Ps})\left(\mathrm{B} \sim 10^{7}-10^{8} \mathrm{G}\right)$ (Bisikalo \& Zhilkin, 2015).

However we have a smooth continuity among the classes, as shown in fig. 7 (Giovannelli \& Sabau-Graziati, 2015).
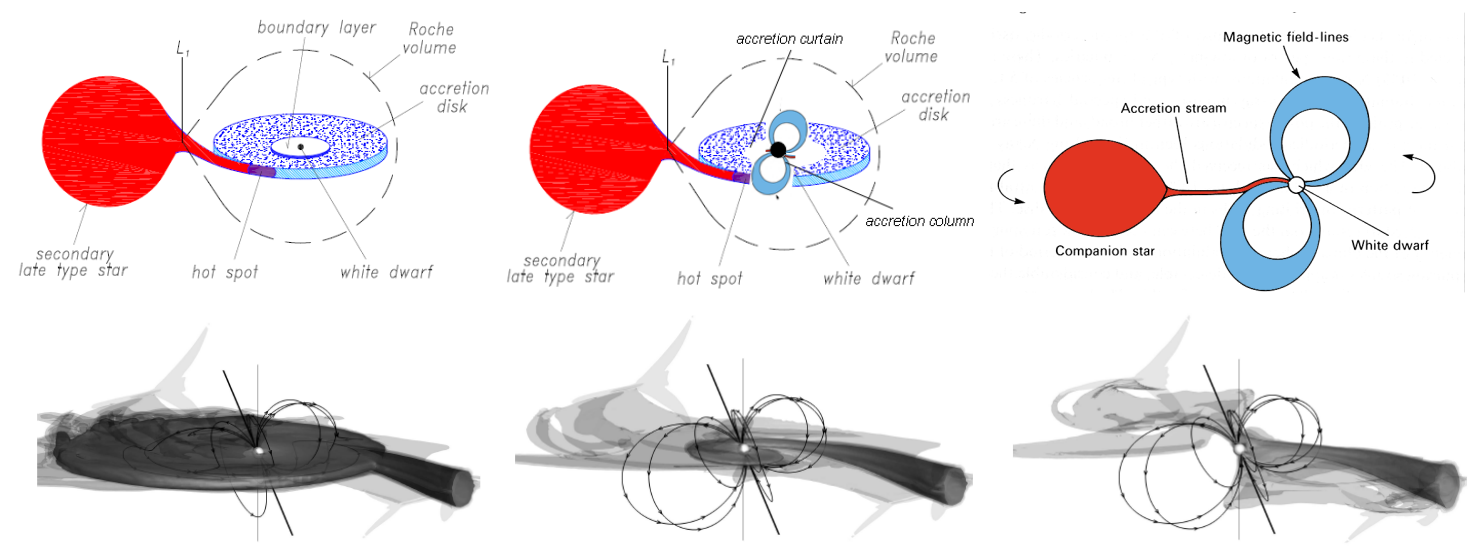

Figure 6: Upper panel: from left to right: sketch of NMCVs, IPs, and Polars (Giovannelli, 2017). Lower panel, from left to the right: non-magnetic $\mathrm{CV}$, intermediate polar, and polar. The 3D MHD simulations were obtained with $\mathrm{B}=10^{5}, 10^{6}$, and $10^{7} \mathrm{G}$, respectively (Bisikalo \& Zhilkin, 2015).

Indeed, taking into account the average values of magnetic field intensity and orbital periods for polars and IPs, and the minimum and maximum value for both parameters ( $\mathrm{B}$ and $\mathrm{P}_{\text {orb }}$ ), it is possible to construct a very interesting plot (Fig. 7) that shows the evident continuity between the two classes of MCVs. Such a continuity has been noted by Schmidtobreick \& Tappert $(2014,2015)$ : $\mathrm{CVs}$ evolution is driven by angular momentum loss; as consequence $\mathrm{P}_{\text {orb }}$ decreases. All long $\mathrm{P}_{\text {orb }}$ CVs cross SW Sex regime before entering in the "period gap". Therefore SW Sex phenomenon is an evolutionary stage in the life of CVs (e.g. Rodriguez-Gil, 2003).

The nature in all its manifestations shows continuity. Then we have to abandon the "convenient method" of thinking everything in watertight compartments and to go toward a general model for compact accreting stars, like was done by Vladimir Lipunov and collaborators when they developed the "Scenario Machine".

Starting from the trivial definition of X-ray Binary Systems (XRBs): they are binary systems emitting X-rays, a natural question arises. Are these systems governed by few physical parameters independent of their nature? The answer is positive. Indeed, High Mass XRBs (HMXRBs), Low Mass XRBs (LMXRBs), Anomalous X-ray Pulsars (AXPs), and Cataclysmic Variables (CVs) can be considered as gravimagnetic rotators: a body with mass $\mathrm{M}$, having a magnetic moment $\vec{\mu}$, 
rotating with rotational velocity $\vec{\omega}$, being the two axis not necessarily coincident, as sketched in Fig. 8 (Giovannelli, 2016). Introducing a physical parameter, $y=\dot{M} / \mu^{2}$, named gravimagnetic parameter, all the gravimagnetic rotators are contained in a plane $\log \mathrm{P}_{\text {spin }}$ vs Log y (Lipunov, 1987; Lipunov \& Postnov, 1988).

The Scenario Machine (Monte Carlo simulations of binary evolution) permits to build up the complete picture of all possible evolutionary stages of binaries in the Galaxy. The basic evolution equation (1.1) used for 500,000 systems containing magnetized stars provided the results contained in the plane $\log \mathrm{P}_{\text {spin }}-\log \mathrm{y}$, reported in the upper panel of Fig. 9. $\mathrm{P}_{\text {spin }}$ is expressed in seconds and the gravimagnetic parameter is expressed in unit of $10^{-42} \mathrm{~g} \mathrm{~s}^{-1} \mathrm{G}^{-2} \mathrm{~cm}^{-6}$. The symbols used for the different types of binaries are explained in the lower panel of Fig. 9. The definition of the characteristic radii can be found in the paper by Lipunov (1987).

$$
\frac{\mathrm{d} \mathrm{I} \omega}{\mathrm{dt}}=\dot{\mathrm{M}} K_{\mathrm{su}}-\frac{\kappa_{\mathrm{t}} \mu^{2}}{\mathrm{R}_{\mathrm{t}}^{3}}
$$

where:

$\mathrm{K}_{\mathrm{su}}=$ specific angular momentum applied by the accretion matter to the rotator;

$\mathrm{K}_{\mathrm{su}}=\sqrt{\mathrm{GM}_{\mathrm{x}} \mathrm{R}_{\mathrm{d}}}$ for Keplerian disk accretion;

$\mathrm{K}_{\mathrm{su}}=\eta_{\mathrm{t}} \Omega R_{\mathrm{g}}^{2}$ for wind accretion in a binary;

$\mathrm{K}_{\mathrm{su}} \sim 0$ for a single magnetic rotator;

$\mathrm{R}_{\mathrm{d}}=$ radius of the inner disk edge;

$\Omega=$ rotational frequency of the binary system;

$\eta_{\mathrm{t}}=1 / 4 \quad$ (Illarionov \& Sunyaev, 1975);

$\kappa_{\mathrm{t}}=$ dimensionless factor;

$\mathrm{R}_{\mathrm{t}}=$ characteristic radius;

$\dot{M}=$ accretion rate in different regimes.

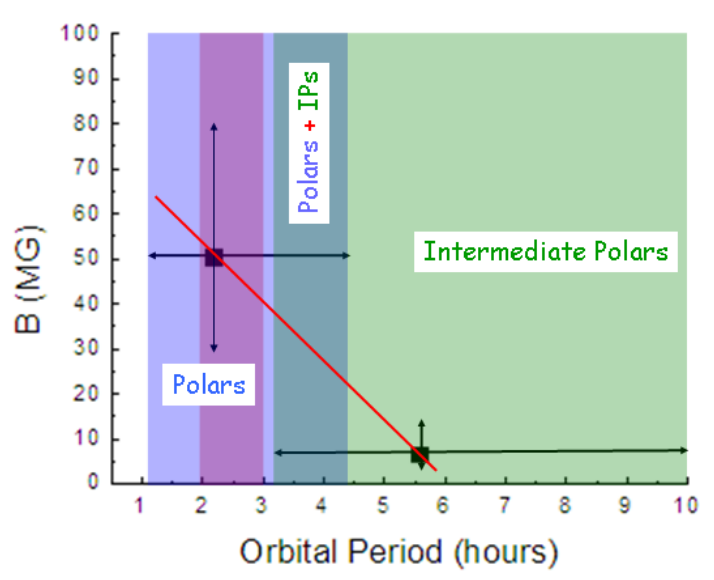

Figure 7: Magnetic field intensity versus orbital period for MCVs. Polars and IPs are contained in the light blue and light green rectangles, respectively. Violet rectangle indicates the so-called "period gap". Cyan-50 rectangle represents the intersection between the Polars and IPs (adopted from Giovannelli \& Sabau-Graziati, 2015). 


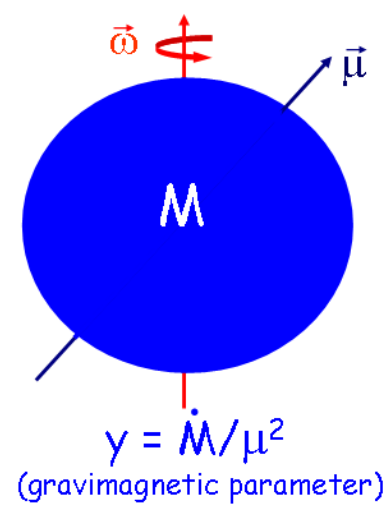

Figure 8: Gravimagnetic rotator: a body with mass $M$, having a magnetic moment $\vec{\mu}$, rotating with rotational velocity $\vec{\omega}$ (adopted from Giovannelli, 2016). The parameter $\mathrm{y}=\dot{\mathrm{M}} / \mu^{2}$ is called gravimagnetic parameter (Lipunov, 1987; Lipunov \& Postnov, 1988).

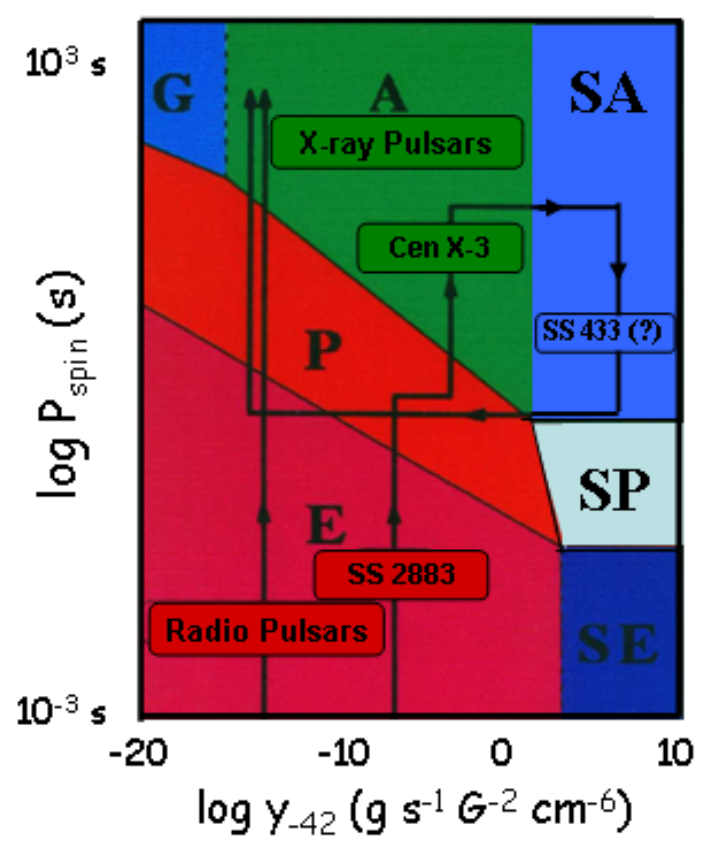

The rotator classification

\begin{tabular}{lll}
\hline Designation & Name & Physical sense \\
\hline E & Ejector & $R_{s t}>\max \left\{R_{G}, R_{l}\right\}$ \\
P & Propeller & $R_{c}<R_{s t} \leq \max \left\{R_{G}, R_{l}\right\} \quad \dot{M}_{c} \leq \dot{M}_{c r}$ \\
A & Accretor & $R_{s t} \leq R_{G}$ and $R_{s t} \leq R_{c} \quad$ \\
G & Georotator & $R_{G}<R_{s t} \leq R_{c}$ \\
M & Magnetor & $R_{s t}>a$ and $R_{c}>a$ \\
SE & Superejector & $R_{s t}>R_{t}$ \\
SP & Superpropeller & $R_{c}<R_{s t} \leq R_{l} \quad \dot{M}_{c}>\dot{M}_{c r}$ \\
SA & Superaccretor & $R_{s t} \leq R_{c}$ and $R_{s t} \leq R_{G}$ \\
\end{tabular}

Figure 9: Upper panel: distribution of magnetic rotators in the plane "Spin Period" versus "Gavimagnetic Parameter" (adopted from Giovannelli, 2016 after Lipunov, 1995); lower panel: classification of rotators (Lipunov, 1987). 


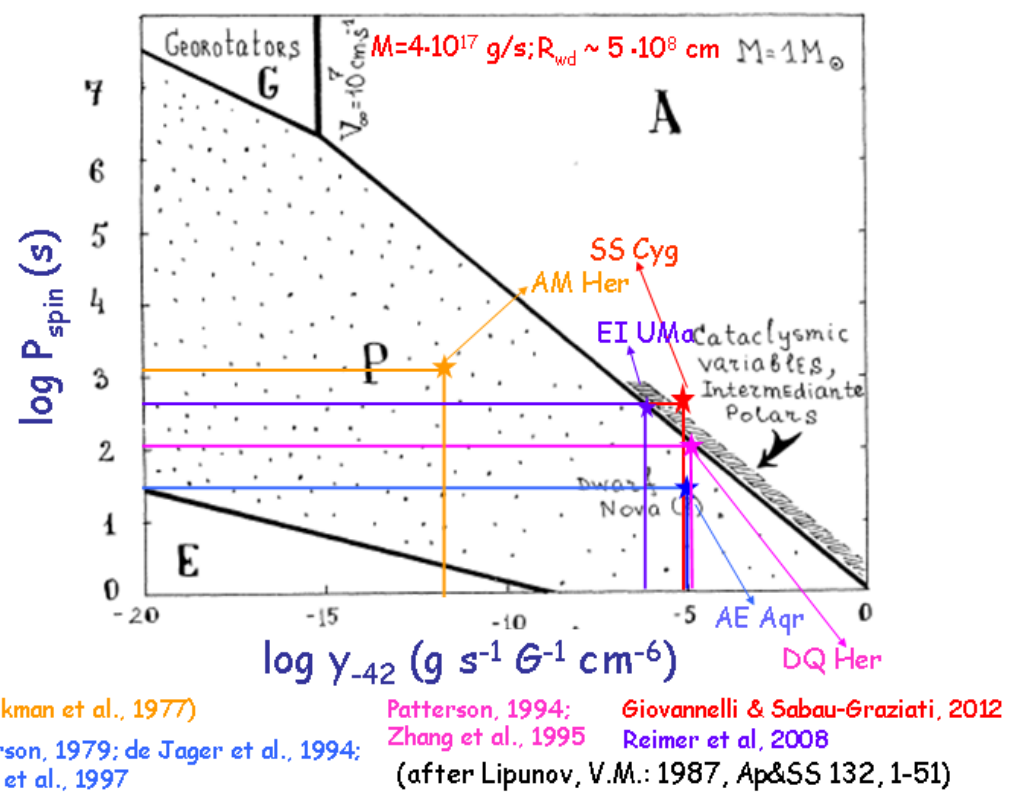

Figure 10: Lipunov's diagram around the zones of propellers and accretors from the scenario machine (adopted from Giovannelli \& Sabau-Graziati, 2015a, after Lipunov, 1987).

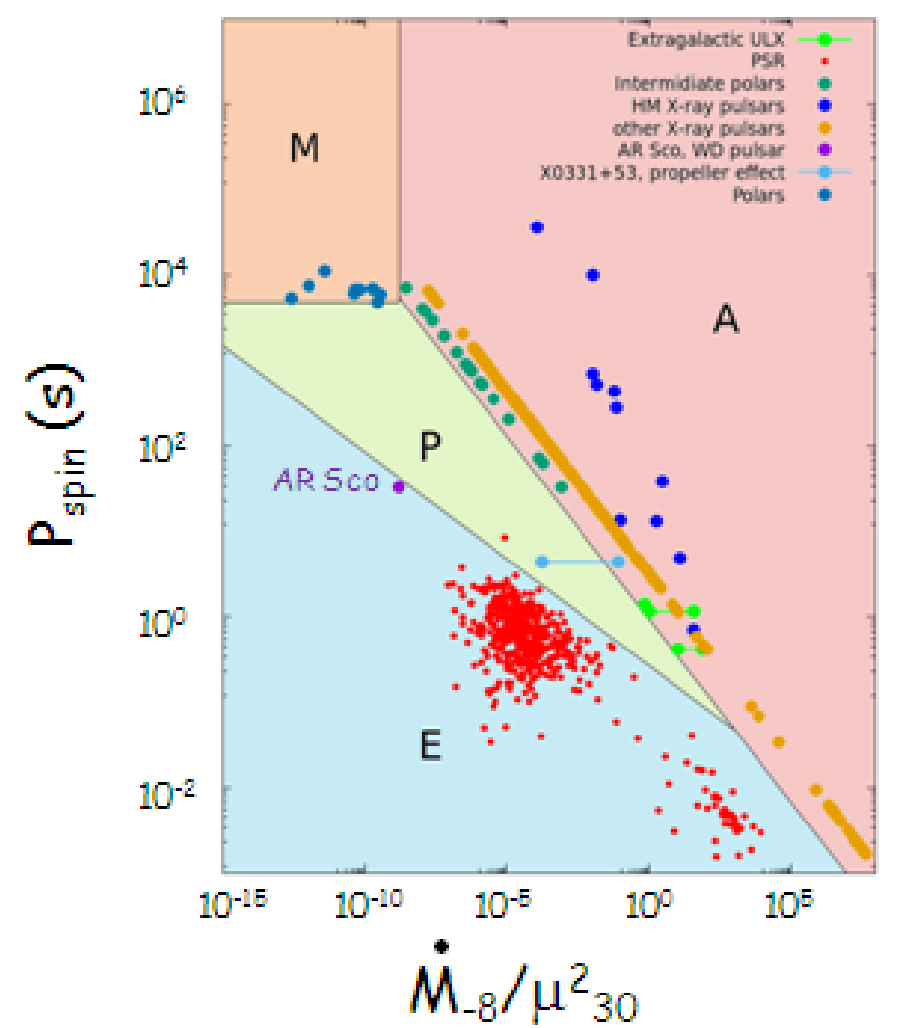

Figure 11: Lipunov's updated diagram from the scenario machine (adapted from Lipunov, 2018). 
Just to illustrate the power of the "Scenario Machine", Figure 10 reports the zoom of the part of the diagram where are reported several cataclysmic variables in the zone of the propeller or accretor depending on the magnetic field intensity at the white dwarf surface and the relative references.

The extraordinary discovery of the first white dwarf pulsar, AR Sco $\left(\mathrm{P}_{\text {orb }}=3.57 \mathrm{~h} ; \mathrm{P}_{\text {spin }}=\right.$ $1.97 \mathrm{~m} ; 0.81 \mathrm{M}_{\odot}<\mathrm{M}_{1} \lesssim 1.29 M_{\odot} ; 0.28 \mathrm{M}_{\odot}<M_{2} \lesssim 0.45 M_{\odot}$ ) (Marsh et al., 2016), and the strong linear polarization $(\lesssim 40 \%)$ variable with $\mathrm{P}_{\text {orb }}$ and $\mathrm{P}_{\text {spin }}$ and beat period detected by Buckley et al. (2017) suggested to Lipunov (2018) to update his diagram $\log \mathrm{P}_{\text {spin }}$ vs $\log \mu$. Indeed, the pulsed luminosity of AR Sco is powered by the spin-down of the rapidly-rotating WD which is highly magnetised $\lesssim 500$ MG. Figure 11 shows such an updated diagram, where the position of AR Sco is reported. It lies just in the ejector zone together with the pulsars.

\subsection{Composition of Our Universe}

The Universe manifests not only through Electromagnetic Radiation but also through Astroparticles, including Neutrinos and Gravitational Waves (GVs).

Since each cosmic source is variable at different levels both in time and intensity, Multifrequency Observations (possibly Simultaneous) are Fundamental in Photonic Astrophysics and Particle Astrophysics. There are many problems in performing Simultaneous Multifrequency, Multisite, Multiinstrument, Multiplatform Measurements due to: (i) objective technological difficulties; (ii) sharing common scientific objectives; (iii) problems of scheduling and budgets; (iv) politic management of science. All these kind of measurements converge in what is now called Multimessenger Astrophysics, after the detection of gravitational wave events (GWEs) and the search for the electromagnetic counterparts of such events.

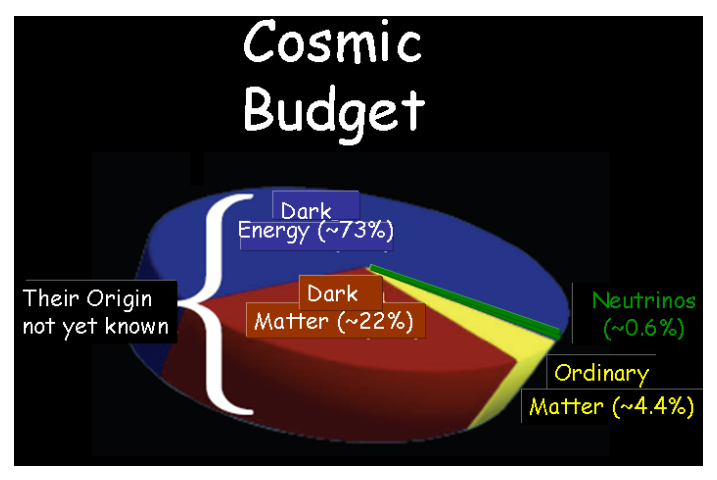

Figure 12: A Sketch of the Cosmic Budget.

The composition of the Universe is poorly known, as shown in Fig. 12. Only $\sim 4.4 \%$ of ordinary matter, $\sim 0.6 \%$ of neutrinos, $\sim 22 \%$ of Dark Matter (DM), and $\sim 73 \%$ of dark Energy (DE). With the detection of GWEs a new window to the Universe has been opened.

As we can see looking at the Fig. 12, we know - and not very well - only $\sim 5 \%$ of our Universe. The one million dollars question is how to improve our knowledge, The answer can be obtained by using Big Experiments (space- and ground-based), and Small Experiments (space- and ground-based). 


\subsubsection{Big experiments}

Several examples of big experiments are:

- GAIA (distances of billions stars);

- Cherenkov Telescope Array (CTA);

- European-Extreme Large Telescope: E-ELT (the next generation ground telescope);

- Canadian Hydrogen Intensity Mapping Experiment (CHIME) (Fast Radio Bursts);

- eASTROGAM ( $200 \mathrm{keV-2} \mathrm{GeV,} \mathrm{High} \mathrm{Sensitivity);}$

- THESEUS (HE transient phenomena, High Sensitivity);

- James Webb Space Telescope: JWST (the next generation space telescope);

- The GAMMA-400 gamma-ray telescope (high angular and energy resolutions);

- The Large Hadron Collider (LHC) (the world's largest and most powerful particle accelerator);

- The BICEP (Background Imaging of Cosmic Extragalactic Polarization) and the Keck Array.

We will discuss later in the section 5 the main characteristic of these experiments.

\subsubsection{Small experiments}

In this short excursion about the tools necessary for an advance of our knowledge of the physics of the Universe, we cannot omit the extreme importance of small experiments, like those Space-based: small-, mini-, micro-, nano-, and cube-satellites, and those Ground-based: smalltelescope, and Robotic-telescopes.

Castro-Tirado (2010a) in his review "Robotic Autonomous Observatories: A Historical Perspective" presented a historical introduction to the field of Robotic Astronomy, discussing the basic definitions, the differing telescope control operating systems, observatory managers, as well as a few current scientific applications in that time.

The number of automatic astronomical facilities worldwide continues to grow, and the level of robotisation, autonomy, and networking is increasing as well. This has a strong impact in many astrophysical fields, like the search for extrasolar planets, the monitoring of variable stars in our Galaxy, the study of active galactic nuclei, the detection and monitoring of supernovae, and the immediate followup of high-energy transients such as gamma-ray bursts (Castro-Tirado, 2008, 2010b).

The number of Robotic Autonomous Observatories (RAOs) has rapidly grown. Figure 13 shows the location of more than 100 RAOs worldwide (Castro Cerón, 2011). They are providing excellent results which should be impossible to obtain with the larger telescopes subject to strict scheduling, and in any case not available for long term runs of observations.

The most important news about the many scientific results obtained with the RAOs can be found in the proceedings of the series of Workshops on Robotic Autonomous Observatories (Bloom, 


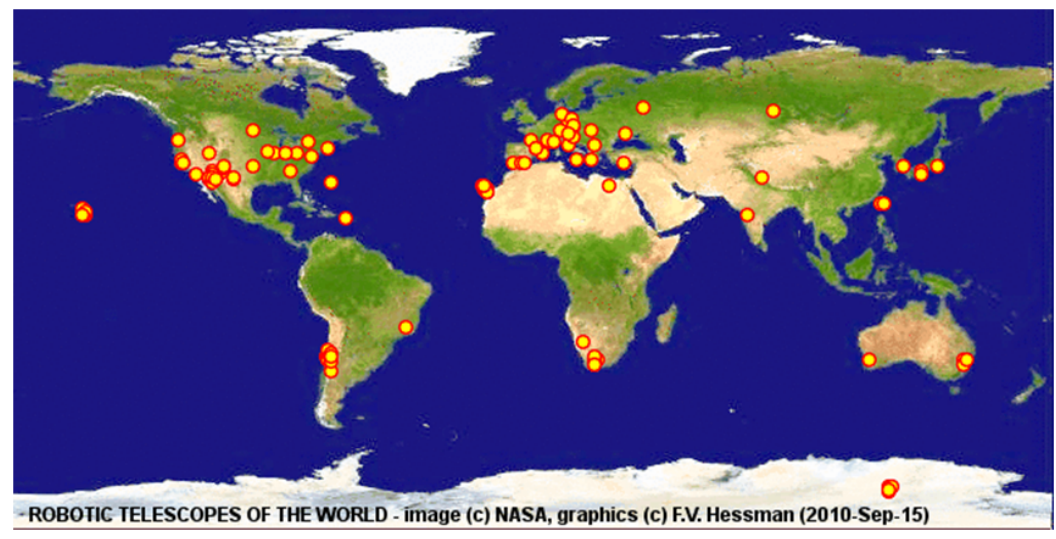

Figure 13: The Robotic Autonomous Observatories worldwide (adopted from Castro Cerón (2011) after Hessman (2001a,b).

Castro-Tirado, Hanlon \& Kotani, 2010; Guziy, Pandey, Tello \& Castro-Tirado, 2012; Tello, Riva, Hiriart \& Castro-Tirado, 2014; Caballero-García, Pandey, Hiriart \& Castro-Tirado, 2016).

Just for giving to the reader a short panorama about the many small ground- and space-based experiments, not necessarily autonomous, we list the following:

a) MITSuME (Multicolor Imaging Telescope for Survey and Monstrous Explosions) has been built to perform Multi-color photometry of NIR/optical afterglow covering the wavebands from $\mathrm{K}_{s}$ to g' allowing the photometric redshift measurements up to $\mathrm{z} \sim 10$. Two $50 \mathrm{~cm}$ optical telescopes are built at Akeno, Yamanashi in eastern Japan, and at OAO, Okayama in western Japan. Each telescope has a Tricolor Camera, which allows us to take simultaneous images in $\mathrm{g}^{\prime}, \mathrm{R}_{c}$, and $\mathrm{I}_{c}$ bands. These telescopes respond automatically to GCN alerts and start taking series of tricolor images, which are immediately processed through the analysis pipeline on site. The pipeline consists of source finding, catalog matching, sky coordinates mapping to the image pixels, and photometry of the found sources. An automated search for an optical counterpart is performed. While waiting for GRBs, the MITSuME Telescopes automatically patrol pre-selected interesting objects such as AGNs and galactic transients for multiwavelength studies with Fermi (GLAST) and MAXI (Shimokawabe et al., 2009).

b) The CHASE (CHilean Automatic Supernova sEarch) project began in 2007 (Pignata et al., 2009) with the goal to discover young, nearby southern supernovae in order to i) better understand the physics of exploding stars and their progenitors, and ii) refine the methods to derive extragalactic distances. During the first four years of operation, CHASE has produced more than 130 supernovae, being the most successful project of its type in the southern hemisphere (Hamuy et al., 2012).

c) The Russian global network of telescopes robot MASTER (Lipunov et al., 2010). MASTER is very fast positioning alert, follow up and survey twin telecopes Global network with own real-time auto-detection software. MASTER goal is One Sky in One Night up to 20-21 mag. The network is spread along the whole world. In the following are reported the MASTER Net Sites: i) MASTER-Amur: Russia, near Blagoveschensk. Blagoveschensk State Pedagogic University. ii) MASTER-Tunka: Russia, near Irkutsk. Applied Physics Institute, Irkutsk State University. iii) 
MASTER-Ural: Russia, near Ekaterinburg, Since 2008. Kourovka Astronomical Observatory, Ural State University. iv) MASTER-Kislovodsk: Russia, Near Kislovodsk. Kislovodsk Solar Station of the Pulkovo Observatory, Sternberg Astronomical Institute, Lomonosov Moscow State University. v) MASTER-SAAO: South Africa, Sutherland, since 2014. South African Astonomical Observatory (SAAO). vi) MASTER-IAC: Spain, Canarias Islands, since 2015 The Instituto de Astrofísica de Canarias (IAC). vii) MASTER-OAFA: Argentina, since 2012 Observatorio Astronomico Felix Aguilar (OAFA), Instituto de Ciencias Astronomicas de la Tierra y del Espacio (ICATE), National University of San Juan. viii) MASTER-Progenitor: Russia, Moscow, Alexander Krylov Observatory, Since 2002.

d) Very small satellites for multifreqyency astrophysics have been discussed by Hudec et al. (2017). About the small satellites we can assist to a strong competition (typically for ESA missions, 60 proposals for 1 satellite), and moreover all the system is affected by funding problems.

The development of the Pico (Cube) and Nanosatellites is running at many Universities, mostly with involvement of students for evident goals of education.

The standard size for a CubeSat is 1 Liter Volume, i.e. $10 \times 10 \times 10 \mathrm{~cm}^{3}$ and typically a weight of $\sim 1.3 \mathrm{~kg}$. Multiple modules are possible, i.e. 3 Units $=3$ modules/units, i.e. $10 \times 10 \times 30 \mathrm{~cm}^{3}$, typically up to 12 Units.

The range of weight of Picosatellites is 0.1-1 kg, Femtosatellites 10-100 g, Nanosatellites 1-10 $\mathrm{kg}$, Microsatellites 10-100 kg.

Recent technological progress allows their use in any field of astrophysics.

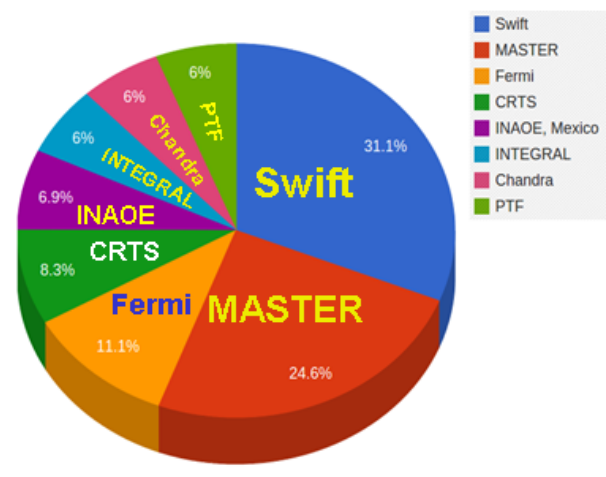

Figure 14: Contribution of different space- and ground-based experiments to the transient alerts in Astronomer's telegrams in the period 2013-2014 (after Buckley, 2015).

Undoubtedly MASTER contributions to transient alerts in Astronomer's telegrams is fundamental. For instance in the period 2013-2014, MASTER contribution is of order 25\% of the total as shown in Fig. 14 (after Buckley, 2015).

Though we have not shown a complete list of small experiments both space- and groundbased, we are able to affirm that small telescopes are unreplaceable tools complementary to larger telescopes and to bigger ground- and space-based multifrequency experiments.

\section{Political management of science}

Thanks to the numerous experiments ground- and space-based, we have collected a huge 
amount of experimental data, the use of which is extremely difficult. This greatly limits the possibility of reaching a synthesis. In contrast, this immense amount of data generates a production of thousands of scientific articles that only in a few cases lead to a real advancement of knowledge.

The science policy, that is now dominating the scientific world, measures the value of a researcher according to: i) the number of "scientific" publications regardless of the real contribution they make to a substantial advancement of knowledge, and ii) the number of citations.

This system foments only the increase of the production of articles at the expense of the synthesis that would be needed, and a chain of "friendly" citations.

And this produces an absurd. Indeed, in order to write a scientific paper a "normal" scientist needs roughly three months of full time work. Thus, four papers in international refereed journals is the upper limit to the yearly publications. We can be generous adding half a dozen of papers presented at the international conferences. Then the new upper limit of publications can be of order of $10 \pm 3 \mathrm{yr}^{-1}$.

Therefore we can derive a severe question to all colleagues who produce more than 13 articles/year. How they do? There are many colleagues who publish more than 100 articles per year!

One of us (FG) remember a repetitive suggestion of Livio Gratton - who was his professor of astrophysics at La Sapienza University of Roma - who felt a true incentive to scientific research: Favour the quality against the quantity! And he was completely right. Indeed he generated - like a supernova expelling heavy elements in the interstellar medium - a number of very famous pupils that pervaded the world of astrophysics. All the readers surely know at least two of them, sketched in Fig. 15 (Giovannelli, 2010).

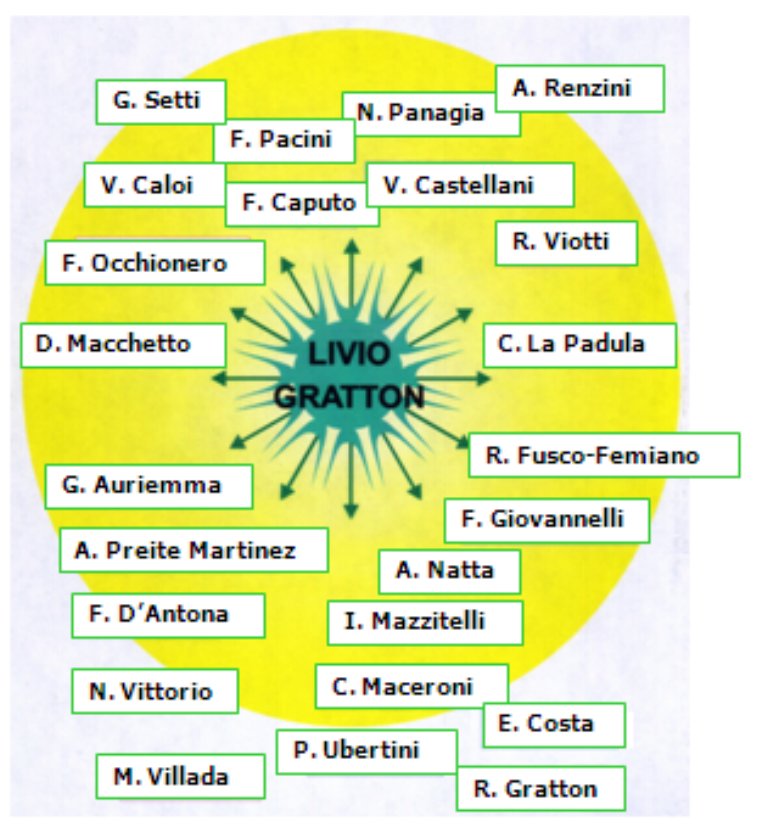

Figure 15: The supernova "Livio Gratton" produced remnant-pupils, all of them rather well known within the international astrophysical community (adapted from Giovannelli, 2010).

Indeed, among the huge amount of papers published in many refereed scientific journals, only very few of them bring a real advancement in the knowledge. Without any pretension of complete- 
ness, we will list few fundamental papers that in our opinion strongly contributed to the advancement of our knowledge of the physics governing our Universe;

- Historically, Baade \& Zwicky (1934a,b) first suggested that a supernova was the result of the transition from a normal star to a neutron star. The essential point (Zwicky, 1939) being that the energy release in such a process is comparable to the change in gravitational potential energy of a star, which collapses from its "normal" size of $\sim 10^{6} \mathrm{~km}$ down to the size of a neutron star of $\sim 10 \mathrm{~km}$. The discovery (almost by chance) of the first X-ray source (Sco X1) (Giacconi, R., Gursky, H., Paolini, F.R., and Rossi, B.B., 1962) accelerated the studies on neutron stars, until that Zel'dovich, Ya, B. \& Guseinov, O.Kh. (1965) suggested the presence of an unseen massive companion in a binary system. The paper by Giacconi et al. (1962) marked the beginning of X-ray astronomy and threw the bases for the future Nobel prize.

- The paper Collapsed Stars in Binary Systems by Guseinov, O.Kh. \& Zel'dovich, Ya, B. (1966). They propose a method for detecting collapsed stars which are members of spectroscopic binary systems. They selected several pairs of systems with invisible companions, in which one may suppose that their components are collapsed stars.

- The paper Energy Emission from a Neutron Star by Franco Pacini (1967). He wrote: "Although there are still many problems concerning the supernovae, there is little doubt that a very dense stellar core has to be left behind after the explosion (at least in some cases). During the contraction of this core, inverse $\beta$ reactions take place and transform most of the nuclei and electrons into neutrons. If the mass of the neutron star does not exceed a critical value of about one or two solar masses, a stable equilibrium situation can be reached with the gas pressure balancing the gravitational force".

- The paper Disk Model of Gas Accretion on a Relativistic Star in a Close Binary System by Nikolai I. Shakura (1972) that introduced the $\alpha$ parametrization of the turbulent viscosity. This paper together with that Black Holes in Binary Systems: Observational Appearance by Shakura \& Sunyaev (1973) marked all the developments in the accretion disk (AD) theory. This latter paper has been cited 9294 times!

- The paper Evidence for strong cyclotron line emission in the hard X-ray spectrum of Hercules X-1 by Trümper et al. (1978) who detected the first cyclotron line from the X-ray binary system Her X-1 at $\sim 58 \mathrm{keV}$ that gave the value of the magnetic field intensity at the surface of the neutron star as $\mathrm{B} \approx 5 \times 10^{12} \mathrm{G}$.

- The Gamma Ray Bursts (GRBs) were discovered in the late 1960s. However, this was not an intentional discovery. They were discovered by the U.S. Vela satellites that were actually built to detect gamma radiation pulses emitted by nuclear weapons tested in Space. Why? Well, the USA suspected that the USSR might attempt to conduct secret nuclear tests after signing the Nuclear Test Ban Treaty in 1963. The historical fundamental papers about GRBs were: (i) Observation of Gamma-Ray Bursts of Cosmic Origin by Klebesadel, Strong \& Olson (1973); (ii) Burst of cosmic gamma-emission from observations on Cosmos 461 by Mazets, Golenetskij \& Il'Inskij (1974); 
- The paper Gamma-Ray Bursters at Cosmological Distances by Bohdan Paczyński (1986). He argued that the three GRBs detected by Mazets, Golenetskii \& Gur'yan (1979) were produced at $\mathrm{z}=1-2$ all due to a single event multiply imaged by a gravitational lens. The required mass of the lens is $10^{10} \mathrm{M}_{\odot}$, just right for a galaxy;

- The paper Discovery of an X-ray afterglow associated with the $\gamma$-ray burst of 28 February 1997 by Costa, E. et al. (1997). They report the detection by the Beppo-SAX satellite of an X-ray "afterglow", associated with the $\gamma$-ray burst of 28 February 1997 (GRB970228) - the first such detection for any $\gamma$-ray burst.

After $\sim 46$ years experience about Multifrequency Astrophysics, we can affirm that: there are many problems in performing Simultaneous Multifrequency, Multisite, Multiinstrument, Multiplatform Measurements due to i) objective technological difficulties; ii) sharing common scientific objectives; iii) problems of scheduling and budgets; iv) political management of science. In our opinion the most critical point is the latter which is moving in a "slippery ground".

A first example of this point is clearly illustrated by the SIXE: Spanish Italian $\mathbf{X}$-ray Experiment (Giovannelli et al., 1993). SIXE was planned as a multifrequency (X-ray, Optical) payload for Long-Term continuous observations of few selected cosmic sources in order to clearly understand the physics governing their behaviour. Later, the phase-A of SIXE was completed thanks to a funding of the Spanish PNIE (Plan Nacional Investigación Espacial), being Principal Investigators Jordi Isern and Franco Giovannelli, and Lola Sabau-Graziati as first Co-I (PNIE-CICYT Report, ESP97-1784-E grant: Isern et al., 1999a; Giovannelli et al., 2002a).

Summaries of that report can be found in several papers later published (Isern et al., 1999b; Giovannelli et al., 1999a,b, 2001, 2002b). Figure 16 shows a summary of the main characteristics of SIXE.

SIXE was submitted to ASI (Agenzia Spaziale Italiana: Italian Space Agency) in order to ask a funding for sharing the costs of the payload with the PNIE, being the launcher (PEGASUS) provided by Spain. No answer at all!!!

After about 26 years from the original idea (Giovannelli, F., Sabau-Graziati, L. et al., 1993), SIXE papers are still read: up till now more than 2000 readings from all the World (source: Research Gate). It is the most read paper in all INAF Institutes!!!

A second example of political management of science is illustrated by the Proposal for a high angular resolution experiment for observing extragalactic sources in the range 20-100 keV made by Giulio Auriemma, Enrico Costa, Franco Giovannelli, Gastone Medici, and Pietro Ubertini (Livio Gratton's disciples) - with particular emphasis to clusters of galaxies, by using their Position Sensitive X-ray Detectors with the Coded Masks - presented at the Italian Extragalactic Astronomy, 1975, April 3 (Auriemma et al. 1975). This proposal was rejected by the Italian Extragalactic Astrophysical Community. Twenty four years later a fundamental paper appeared claiming the detection of hard X-ray emission from Coma cluster (Fusco-Femiano et al., 1999)!!!

With big and small experiments we are going to know better the composition and the laws governing our Universe. However, for this purpose it is much better the use of wisdom besides the knowledge of the physics. 


\section{SIXE (Spanish Italian X-ray Experiment)

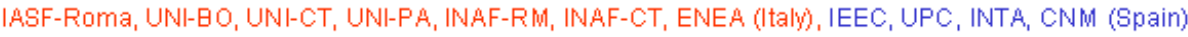
FASE-A successfully concluded: funded by the Spanish PNIE (Plan Nacional Investigación Espacial)

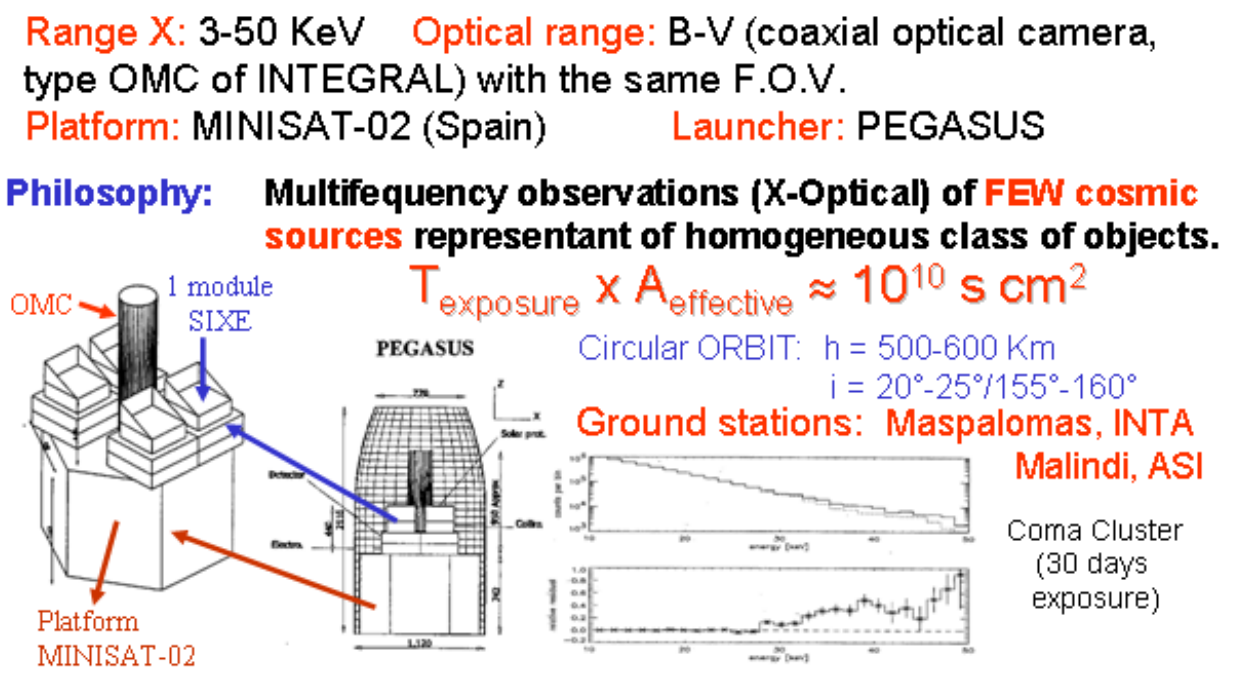

Figure 16: SIXE (Spanish Italian $\mathbf{X}$-ray Experiment): main characteristics.

\section{The use of wisdom in physics}

And now some examples of problems resolved with the help of multifrequency observations and good small quantities of wisdom in physics.

\subsection{X-ray/Be systems}

$\mathrm{X}$-ray/Be systems are formed by a compact star and an optical star. Obviously there is a mutual influence between the two stars. Low-energy (LE) processes influence high-energy (HE) processes and vice versa. Never confuse the effect with the cause. There is a general law in the Universe: Cause and Effect. The Cause generates an Effect and NOT vice versa!

Time-lag between HE events and LE events in disk-fed accreting X-ray binaries (XRBs) has been noted in many systems, but the trigger of the work resulted in a model for explaining in general such a phenomenon (Bisnovatyi-Kogan \& Giovannelli, 2017) was given by Giovannelli \& SabauGraziati (2011) who noted a systematic delay between the relative enhancement in luminosity of the optical Be star - occurring at the periastron passage of the neutron star - and the subsequent X-ray flare in the system HDE 245770/A 0535+26. The model for such a system was developed and corroborated by many events (Giovannelli, Bisnovatyi-Kogan \& Klepnev, 2013: GBK13), and later by events reported in Giovannelli et al. (2015) where also a relationship between $\Delta \mathrm{V}_{\text {mag }}$ of the optical star at the periastron and X-ray intensity $\left(\mathrm{I}_{\mathrm{X}}\right)$ of the 8-day delayed flare was produced.

Briefly, the model based on an accretion disk geometrically thin and optically thick without advection (Shakura \& Sunyaev, 1973; Bisnovatyi-Kogan, 2002) is the following: in the vicinity of periastron the mass flux $\dot{M}$ increases (depending on the activity of the Be star) between $\approx 10^{-8}$ 
and $\approx 10^{-7} \mathrm{M}_{\odot} \mathrm{yr}^{-1}$. The outer part of the accretion disk becomes hotter, therefore the optical luminosity $\left(\mathrm{L}_{\mathrm{opt}}\right)$ increases. Due to large turbulent viscosity, the wave of the large mass flux is propagating toward the neutron star, thus the $\mathrm{X}$-ray luminosity $\left(\mathrm{L}_{\mathrm{x}}\right)$ increases due to the appearance of a hot accretion disk region and due to the accretion flow channeled by the magnetic field lines onto magnetic poles of the neutron star. The time-delay $\tau$ is the time between the optical and X-ray flashes appearance.

It is right to remind that the mechanism proposed by GBK13 for explaining the X-ray-optical delay in A $0535+26 /$ HDE 245770 is based on an enhanced mass flux propagation through the viscous accretion disk. This mechanism, known as UV-optical delay (the delay of the EUV flash with respect to the optical flash) was observed and modeled for cataclysmic variables (e.g. Smak, 1984; Lasota, 2001). Time delays have been detected also in several other X-ray transient binaries. This is the reason that urged Bisnovatyi-Kogan \& Giovannelli (2017) to generalize the aforementioned model, developed for the particular case of A 0535+26/HDE 245770 (Flavia' star). This general model provides the formula (3.1) of the time delay in transient cosmic accreting sources:

$$
\tau=6.9 \frac{\mathrm{m}^{2 / 3} \dot{\mathrm{m}}^{1 / 15}}{\alpha^{4 / 5}\left(\mathrm{~T}_{4}\right)^{28 / 15}}
$$

where:

$\mathrm{m}=\mathrm{M} / \mathrm{M}_{\odot} ; \quad \dot{\mathrm{m}}=\dot{\mathrm{M}} /\left(10^{-8} \mathrm{M}_{\odot} / \mathrm{yr}\right) ; \quad \mathrm{T}_{4}=\mathrm{T}_{0} / 10^{4} \mathrm{~K} ; \quad \alpha=$ viscosity, and

$\mathrm{T}_{0}=$ maximum temperature in optics.

By using this formula it is possible to obtain an excellent agreement between the experimental and theoretical delays found in:

- X-ray/Be system A0535+26/HDE245770: $\tau_{\text {exp }} \simeq 8$ days (GBK13); $\tau_{t h} \simeq 8$ days;

- Cataclysmic variable SS Cygni; $\tau_{\text {exp }}=0.9-1.4$ days (Wheatley, Mauche \& Mattei, 2003); $\tau_{t h} \simeq 1.35$ days;

- Low-mass X-ray binary Aql X-1/V1333 Aql: $\tau_{\exp } \sim 3$ days (Shahbaz et al., 1998); $\tau_{t h} \simeq$ 3.2days

- Black hole X-ray transient GRO J1655-40: $\tau_{\text {exp }} \sim 6$ days (Orosz et al., 1997); $\tau_{t h} \simeq 6.5$ days.

In this general formula the $\alpha$-viscosity parameter plays an important role, and usually it is hard to be determined. However, if the other parameters are known, because experimentally determined, the formula (3.1) can be used for determining $\alpha$, taking into account the experimental delay measured in a certain source.

This general model for the time-lag for disk-fed accreting XRBs is sketched in Fig. 17.

Another example of the use of wisdom is that referred to the X-ray/Be system A 1118-61/Hen 3-640, for which Reig, Fabregat \& Coe (1997) used one single measurement of the equivalent width (EW) of $\mathrm{H}_{\alpha}(89 \AA)$ in their interesting diagram in which a relationship between the $\mathrm{H}_{\alpha}$-EWs and the orbital period $\left(\mathrm{P}_{\mathrm{orb}}\right)$ of $\mathrm{Be} / \mathrm{X}$-ray binaries has been found. The point relative to A 1118-61/Hen 3-640 system (red cross in Fig. 18) is clearly outside of the line best fitting the other data. However, 


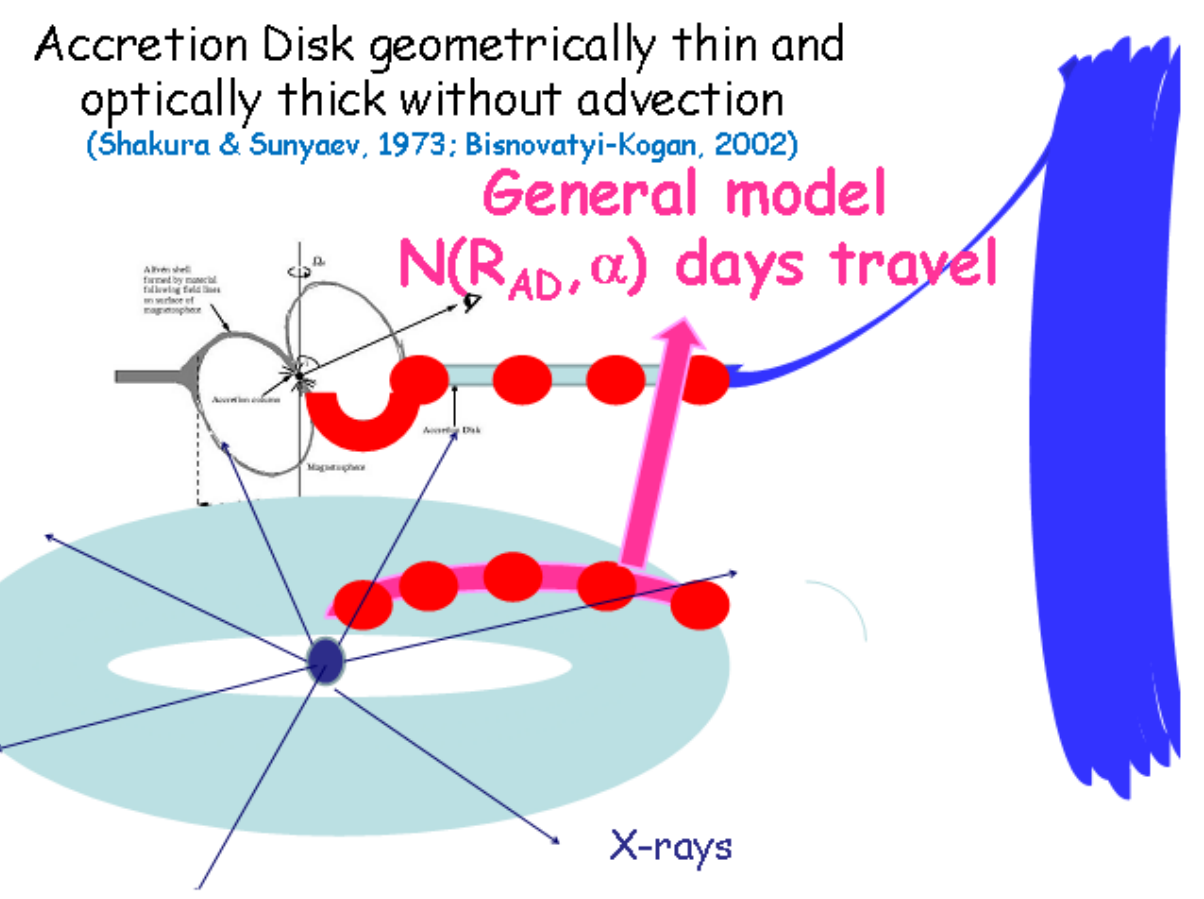

Figure 17: Time-lag general model for disk-fed accreting XRBs (Bisnovatyi-Kogan \& Giovannelli, 2017).

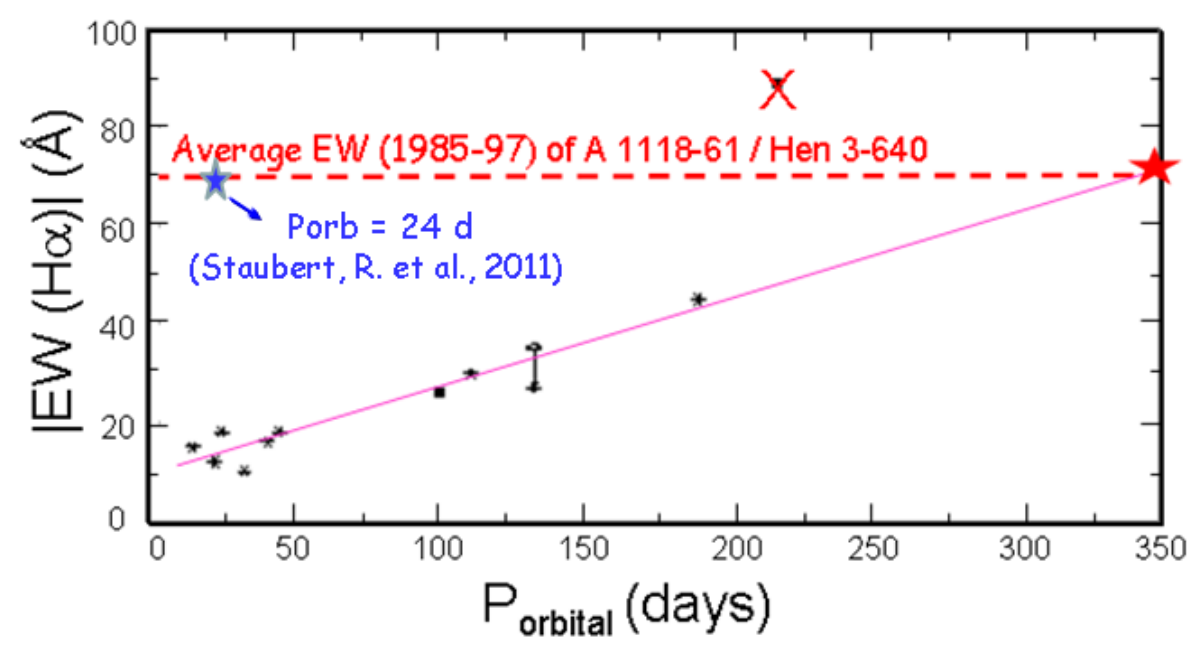

Figure 18: The relationship between $\mathrm{H}_{\alpha}-\mathrm{EW}$ and $\mathrm{P}_{\text {orb }}$ in $\mathrm{X}$-ray/Be systems (after Reig, Fabregat \& Coe, 1997; Villada et al., 1999). 
if we use the average value (from 1985 to 1997) of $\mathrm{H}_{\alpha}-\mathrm{EW}=70 \AA$, like reported in Villada et al. (1999), also the position of the A 1118-61/Hen 3-640 system is well on the line best fitting the data (red star in Fig. 18). Moreover an indication of the possible value of the orbital period $(\sim 350$ days), not yet known, is coming from that diagram. This can help the search for the orbital period of the system around the value of 350 days. However, a value of orbital period of 24 days was reported by Staubert et al. (2011). In our opinion this value is wrong. Indeed if we put such a value in the diagram of Fig. 18 (blue cross) in correspondence with the average value of $\mathrm{H}_{\alpha}-\mathrm{EW}=70 \AA$, it appears completely outside the line best fitting the data.

\subsection{The classical T Tauri star RU Lupi}

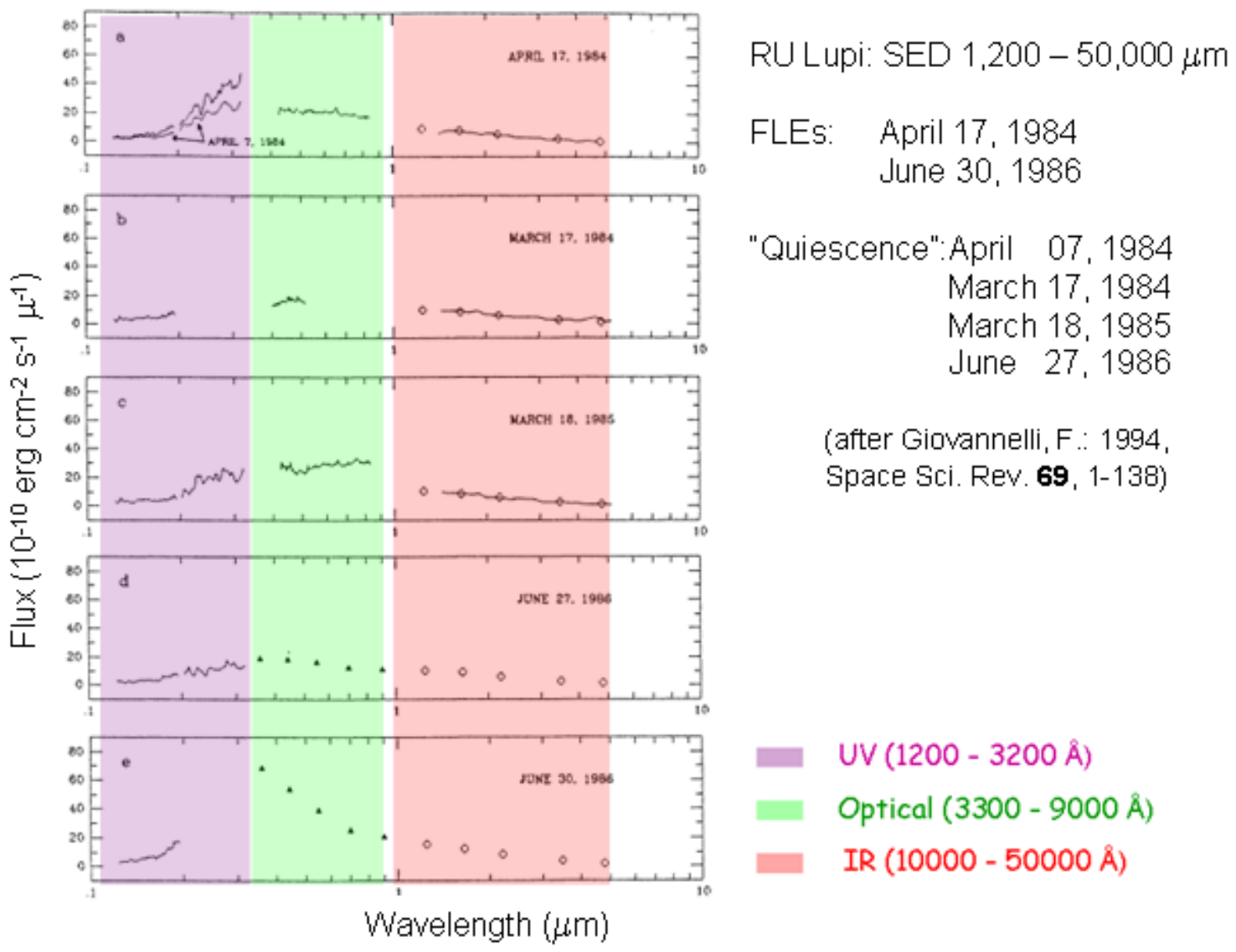

Figure 19: $\operatorname{SED}(1,200-50,000 \mu \mathrm{m})$ of RU Lupi in different epochs (after Giovannelli, 1994).

A long-term (1982-1988) multifrequency program on Classical T Tauri Stars (CTTSs) was developed by an international group led by Franco Giovannelli. The facilities used for such a campaign were the International Ultraviolet Explorer (IUE), the ASTRON X-ray/UV Soviet satellite, and the ESO 0.6-m UBVRI telescope, 1-m IR telescope, 1.5-m telescope for low resolution optical spectroscopy, and 3.6-m telescope for Echelle high resolution spectroscopy.

The results were published in two main papers, the first with the experimental results (Giovannelli et al., 1995), the second with the interpretation of data and modeling (Lamzin et al., 1996). A review paper about RU Lupi was published by Giovannelli (1994). 
One of the main results obtained during the long-term multifrequency program was the simultaneous detection of emissions in different energy bands that allowed to construct the Spectral Energy Distribution (SED) of RU Lupi, as shown in Fig. 19.

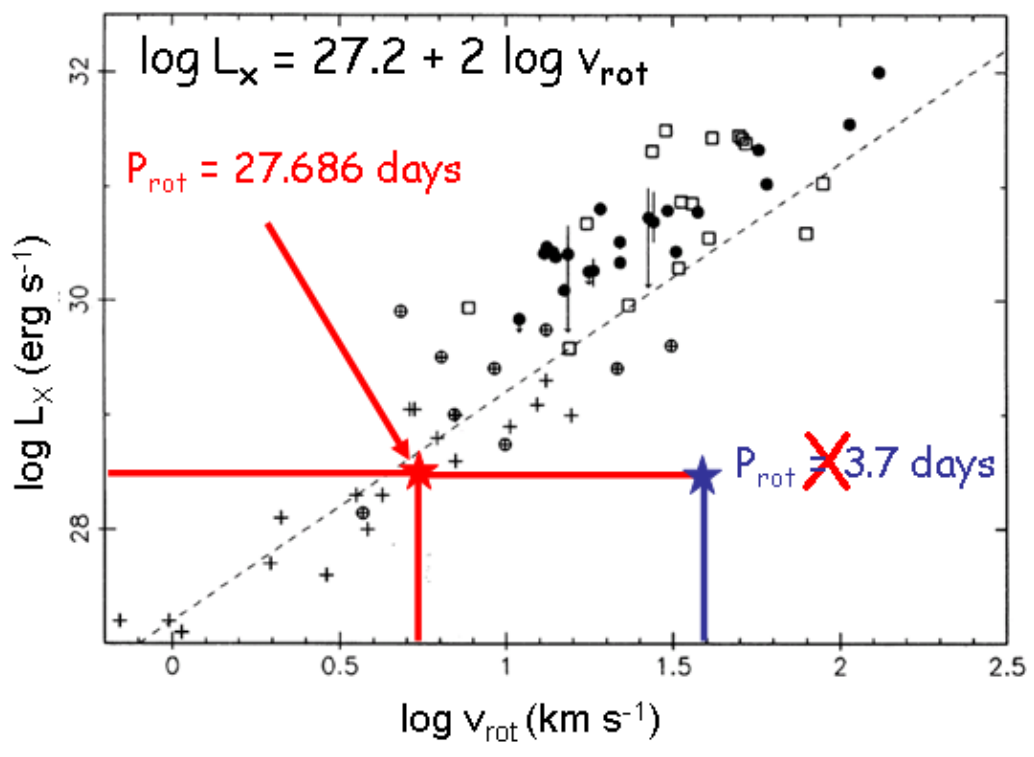

Figure 20: X-ray luminosity vs stellar equatorial velocity for TTS $(\bullet)$, late-type dwarfs $(+)$, dKe-dMe stars $(\oplus)$, and RS CVn systems (square). The right position of RU Lupi is marked with a red cross, and the wrong position is marked with a blue cross (Giovannelli, 1994 after Bouvier, 1990).

In two occasions, RU Lupi showed a strong activity (Flare-Like Events: FLEs), much higher than that in "quiescence". Together with the FLEs reported in the literature, these two FLEs allowed to determine their periodicity: $\mathrm{P}_{\mathrm{FLEs}}=27.686 \pm 0.002$ days (Giovannelli, 1994). This could be the rotational period of RU Lupi. Indeed, if we use the relationship between the X-ray luminosity $\left(\mathrm{L}_{\mathrm{X}}\right)$ versus the rotational velocity $\left(\mathrm{v}_{\mathrm{rot}}\right)$ for $\mathrm{T}$ Tauri stars, late-type dwarfs, dKe-dMe stars, and RS CVn systems (Bouvier, 1990), the position of RU Lupi fits the relationship $\log L_{X}=27.2+2$ $\log v_{\text {rot }}$ if $\mathrm{P}_{\mathrm{FLEs}}=27.686 \pm 0.002$ days is used, instead of using the "wrong" value reported in the literature of 3.7 days - that simply does not exist (Giovannelli et al., 1991) - whose wrong origin is largely commented in the paper by Giovannelli (1994). Figure 20 shows the diagram of the Xray luminosity $\left(\mathrm{L}_{\mathrm{X}}\right)$ versus the rotational velocity $\left(\mathrm{v}_{\mathrm{rot}}\right)$ where the "correct" position of RU Lupi is overlapped with a red cross, and the "wrong" position with the blue cross.

\subsection{Great example of synergy between Astrophysics and History}

Bernd Aschenbach modified Sedov's relation for determining the age of SNRs (Aschenbach, 2016). He used as test the SNR Vela Jr (RX 0852.0-3946) - discovered during the ROSAT AllSky-Survey in X-rays (Aschenbach, 1998) - and he gave an age of $\mathrm{T}_{\text {Aschenbach }} \sim 725 \mathrm{yr}$ - contrary to $\mathrm{T}_{\text {Sedov }} \sim 1714 \mathrm{yr}-$ and a distance of $\sim 386 \mathrm{pc}$.

Historical document (Tatsunokuchi Persecution of Nichiren Daishonin "the Buddha of the last day of the law") supports this result with an exceptional precision: The date of the explosion was 12 September $1271(1 \pm 2$ a.m. - between the hours of the rat and the ox) (Soka Gakkai, 1999). 
How is it possible to affirm that the explosion of the SN Vela Jr happened in that date with a strong precision?

The answer, indeed, can be found in the writings of Nichiren Daishonin. This buddhist monk presented to the public authority the "Risho Ankoku Ron" (Establishing the Correct Teaching for the peace in the country) three times: a strong and clear critic to the behaviour of authority. For this reason he was persecuted and sentenced to death.

At that moment Nichiren was about to be beheaded, a luminous object (full Moon) shot across the sky, brightly illuminating the surroundings. The executioner fell on his face, his eyes blinded. The soldiers were filled with panic. Terrified, the soldiers called off the execution. This happened on the twelfth day of the ninth month of 1271, between the hours of the rat and the ox (11:00 p.m. to 3:00 a.m.). The event culminated $10^{\circ}$ above the horizon, celestial declination $-46^{\circ}$ (position of Vela Jr).

\section{The present situation about the knowledge of the physics of our Universe}

Undoubtedly the advent of new generation experiments ground- and space-based have given a strong impulse for verifying current theories, and for providing new experimental inputs for developing a new physics for going, probably, over the standard model (SM). Recent results coming from Active Physics Experiments (APEs) and Passive Physics Experiments (PPEs) have opened such a new path.

An extensive review on the situation about the knowledge of the physics of our Universe has been recently published by Giovannelli \& Sabau-Graziati (2016c). The reader interested is invited to look at that paper. However, we are obliged to discuss a few topics that, in our opinion, could be useful for a better understanding of the open problems still existing in the modern astrophysics.

\subsection{Active physics experiments}

One of the most exciting results from LHC is the detection of the Higgs boson which is often called "the God particle" because it's said to be what caused the "Big Bang" that created our Universe. Matter obtains mass interacting with Higgs field. Thus, if the Higgs Boson is detected, the Standard Model of Physics would be completed.

The Standard Model of particle physics takes quarks and leptons to be fundamental elementary particles, and describes the forces that govern their interactions as mediated through the exchange of further elementary particles. The exchanged particles are photons in the case of the electromagnetic interaction, $\mathrm{W}$ and $\mathrm{Z}$ bosons in the case of the weak interaction, and gluons in the case of the strong interaction. After the discovery of the $\mathrm{W}$ and $\mathrm{Z}$ bosons in the early 1980s, the elucidation of the mechanism by which they acquire mass became an important goal for particle physics. Within the Standard Model the $\mathrm{W}$ and $\mathrm{Z}$ bosons have masses generated via the symmetry breaking Englert-Brout-Higgs-Guralnik-Hagen-Kibble mechanism, proposed in 1964 and giving rise to a massive scalar particle, the Standard Model Higgs boson (Jakobs \& Seez, 2015).

The hunt to Higgs boson started a few years ago with the most powerful accelerators constructed in the world, in particular with the different experiments of the LHC. These experiments can provide information about the first moment of the life of the Universe. LHC is a complementary tool for HE observatories looking directly to the Universe. 
The Higgs boson discovery was announced by the ATLAS (A Toroidal LHC ApparatuS) and CMS (Compact Muon Solenoid) collaborations on 4th July 2012. Evidence for a new particle with the mass of about $125 \mathrm{GeV}$ and the properties of the Standard Model Higgs boson.

From ATLAS results, a $5.0 \sigma$ excess at $\sim 126.5 \mathrm{GeV}$ has been detected. This value is compatible with the expected mass of Higg's boson (Gianotti, 2012; Aad et al., 2012). The Compact Muon Solenoid (CMS) experiment at LHC detected a new boson at $125.3 \pm 0.6 \mathrm{GeV}$ with $4.9 \sigma$ significance (Incandela, 2012; The CMS Collaboration, 2012). This result, together with that from ATLAS, if confirmed, would complete the SM of physics.

Thanks to collisions at $13 \mathrm{TeV}$ the experiment Large Hadron Collider beauty (LHCb) at LHC detected a new particle: the Pentaquark. The existence of the pentaquark was theoretically suggested since 1960-ies (Gell-Mann, 1964). Pentaquark gives a new way for the combination of the quarks that are the fundamental constituents of neutrons and protons (Cardini, 2015; Aaij et al., 2015).

\subsection{Passive physics experiments}

One of the most important questions still open is the search for experimental proof of the inflation. The expansion is thought to have been triggered by the phase transition that marked the end of the preceding grand unification epoch at $\approx 10^{-36} \mathrm{~s}$ after the Big Bang. It is not known exactly when the inflationary epoch ended, but it is thought to have been between $\approx 10^{-33}$ and $\approx 10^{-32} \mathrm{~s}$ after the Big Bang. The experimental proof of the inflation could come from measurements of Cosmic Microwave Background (CMB) polarization. Winstein $(2007,2009)$ discussed the problem of CMB polarization in the following decade.

We know from the theory that linear polarization of the CMB photons is induced via Thomson scattering by quadrupole anisotropy at recombination that occurred at $\mathrm{z} \sim 1100$ corresponding to $\mathrm{t} \sim 1.2 \times 10^{13} \mathrm{~s}$ after the Big Bang. In turn, quadrupole anisotropy is induced by: i) density perturbations (scalar relics of inflation) producing a curl-free polarization vector field (E-modes); ii) gravitational waves (tensor relics of inflation) producing both curl-free and curl-polarization fields (B-modes).

No other sources for a curl-polarization field on the CMB at large angular scales exist. Thus, B-modes are a clear signature of inflation (e.g. de Bernardis, 2014).

Recently the collaboration of the BICEP2 experiment claims the detection of E-mode (Crites et al., 2015) and B-mode polarization of the CMB at at 7.0 $\sigma$ significance (Ade et al., 2015). If Bmode polarization would be confirmed, the inflationary model of the Universe would be definitively confirmed. However, big discoveries need big confirmations. For a robust detection of B-modes, independent measurements and precise measurements of polarized foregrounds are mandatory.

Indeed, a key element to the primordial interpretation advanced by the BICEP2 team was excluding an explanation based on polarized thermal dust emission from our galaxy (Bucher, 2015a,b). An independent analysis cast doubt on the BICEP2 claim (Flauger, Hill \& Spergel, 2014). In September 2014 the Planck team published a paper on the level of polarized dust emission measured across the whole sky, and in particular in the BICEP2 field (Planck Collaboration, 2014). This work also extrapolated the polarized dust signal seen in the Planck $353 \mathrm{GHz}$ map (a frequency in the Wien tail of the CMB blackbody where dust dominates) down to $150 \mathrm{GHz}$ and 
reached the conclusion that the BICEP B mode signal could be entirely explained by polarized dust emission although a primordial B mode contribution could not be ruled out.

However, the theory of inflation is criticized by Ijjas, Steinhadt \& Loeb (2013) after Planck2013 results. They suggest that the origin of the Universe is not the Big Bang, but could be a "bouncing" Universe that does not need the inflation. Membrane-Universes that clashed endlessly could be a "plausible" alternative model for the Universe (Erickson et al., 2007; Steinhardt, Turok \& Starkman, 2008). Cyclic models of the universe have the advantage of avoiding initial conditions problems related to postulating any sort of beginning in time (Ijjas, 2016).

For all these reasons is even more important to find an experimental proof of the Inflation.

\subsection{Confirmation of the Theory of General Relativity}

In the last few years two further experimental results confirmed the validity of the theory of General Relativity (GR theory).

\subsubsection{Gravitational lenses}

Renn, Sauer \& Stachel (1997) published a historical reconstruction of some of Einstein's research notes dating back to 1912 . These notes reveal that he explored the possibility of gravitational lensing 3 years before completing his general theory of relativity. On the basis of preliminary insights into this theory, Einstein had already derived the basic features of the lensing effect. When he finally published the very same results 24 years later, it was only in response to prodding by an amateur scientist.

Kochanek (2003) discussed "The whys and hows of finding 10,000 lenses", mentioning the first radio lens survey - the MIT - Green Bank survey (MG) - that found lenses by obtaining Very Large Array (VLA) snapshot images of flux-limited samples of $5 \mathrm{GHz}$ radio sources. The Hubble Space Telescope (HST), and Chandra observations (e.g. Dai \& Kochanek, 2005) showed without any doubt that the gravitational lensing is operating.

Gravitational lensing is widely and successfully used to study a range of astronomical phenomena, from individual objects, like galaxies and clusters, to the mass distribution on various scales, to the overall geometry of the Universe (Williams \& Schechter, 1997). They describe and assess the use of gravitational lensing as "gold standards" in addressing one of the fundamental problems in astronomy, the determination of the absolute distance scale to extragalactic objects, namely the Hubble constant.

Several papers have been published about the strong gravitational lensing (e.g. Tyson, Kochanski \& Dell'Antonio, 1998; Tyson, 2000 and references therein), and the weak gravitational lensing (Wittman et al., 2000). A review on "Gravitational Lenses" have been published by Blandford \& Kochanek (2004). A book on "Gravitational Lensing: Strong, Weak and Micro" was published by Meylan et al. (2006). Winn, Rusin \& Kochanek (2004) reported the most secure identification of a central image, based on radio observations of PMN J1632-0033.

Therefore, a further dowel supports the GR theory.

It is important to mention the paper Resource Letter GL-1: Gravitational Lensing by Treu, Marshall \& Clowe (2012). This Resource Letter provides a guide to a selection of the literature on gravitational lensing and its applications. Journal articles, books, popular articles, and websites 
are cited for the following topics: foundations of gravitational lensing, foundations of cosmology, history of gravitational lensing, strong lensing, weak lensing, and microlensing.

Indeed, each Resource Letter focuses on a particular topic and intends to help teachers to improve course content in a specific field of physics or to introduce nonspecialists to this field.

\subsubsection{Gravitational waves}

The Universe that contains by definition all the matter or all the energy available showed one important event that was possible to be detected on the Earth. This event was a further direct experimental demonstration of the validity of the GR theory. Indeed, on September 14, 2015 at 09:50:45 UTC the two detectors of the Laser Interferometer Gravitational-Wave Observatory (LIGO) simultaneously observed a transient gravitational-wave signal. It matches the waveform predicted by GR theory for the inspiral and merger of a pair of black holes and the ringdown of the resulting single black hole. The signal was observed with a significance $\geq 5.1 \sigma$ (Fig. 21). The source lies at a luminosity distance of $410_{-180}^{+160} \mathrm{Mpc}$ corresponding to a redshift $\mathrm{z}=0.090_{-0.04}^{+0.03}$. In the source frame, the initial black hole masses are $36_{-4}^{+5} \mathrm{M}_{\odot}$ and $29 \pm 4 \mathrm{M}_{\odot}$, and the final black hole mass is $62 \pm 4 \mathrm{M}_{\odot}$ with $3.0 \pm 0.5 \mathrm{M}_{\odot} \mathrm{c}^{2}$ radiated in gravitational waves. All uncertainties define $90 \%$ credible intervals. These observations demonstrate the existence of binary stellar-mass black hole systems. This is the first direct detection of gravitational waves and the first observation of a binary black hole merger (Abbott et al., 2016a).

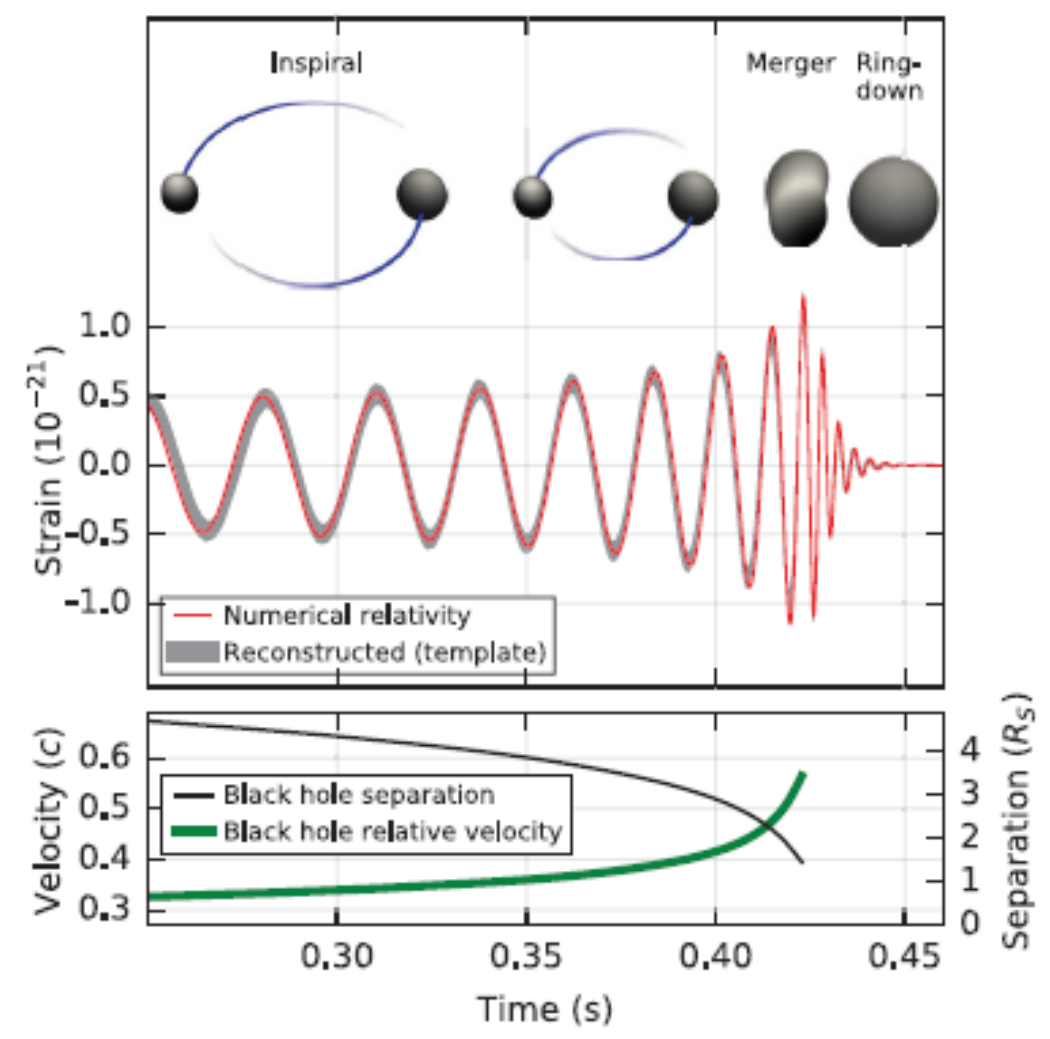

Figure 21: The GW150914 event. Top: estimated gravitational-wave strain amplitude. Bottom: the Keplerian effective black hole separation in units of Schwarzschild radii (adopted from Abbott et al., 2016a). 
Abbott et al. (2016b) reported the second observation of a gravitational-wave signal produced by the coalescence of two stellar-mass black holes. The signal, GW151226, was observed by the twin detectors of the LIGO on December 26, 2015 at 03:38:53 UTC. The signal was detected at significance $\geq 5 \sigma$. The inferred source-frame initial black hole masses are $14.2_{-3.7}^{+8.3} \mathrm{M}_{\odot}$ and 7.5 \pm $2.3 \mathrm{M}_{\odot}$, and the final black hole mass is $20.8_{-1.7}^{+6.1} \mathrm{M}_{\odot}$. One finds that at least one of the component black holes has spin greater than 0.2 . This source is located at a luminosity distance of $440_{-190}^{+180}$ Mpc corresponding to a redshift $\mathrm{z}=0.09_{-0.04}^{+0.03}$. All uncertainties define a $90 \%$ credible interval. This second gravitational-wave observation provides improved constraints on stellar populations and on deviations from the GR theory.

For these detections of gravitational waves - first predicted by Einstein 100 years ago - Rainer Weiss, Barry Barish \& Kip Thorne have been awarded the 2017 Nobel prize in physics.

Abbott et al. (2016c) present a possible observing scenario for the Advanced LIGO (aLIGO) and Advanced Virgo gravitational-wave detectors over the next decade, with the intention of providing information to the astronomy community to facilitate planning for multimessenger astronomy with gravitational waves.

Gravitational waves provide a revolutionary tool to investigate yet unobserved astrophysical objects. Especially the first stars, which are believed to be more massive than present-day stars, might be indirectly observable via the merger of their compact remnants. An interesting paper by Hartwig et al. (2016) developed a self-consistent, cosmologically representative, semi-analytical model to simulate the formation of the first stars. They estimated the contribution of primordial stars to the merger rate density and to the detection rate of the aLIGO. Owing to their higher masses, the remnants of primordial stars produce strong GW signals, even if their contribution in number is relatively small. They found a probability of $\geq 1 \%$ that the current detection GW150914 is of primordial origin. The higher masses of the first stars boost their GW signal, and therefore their detection rate. Up to five detections per year with aLIGO at final design sensitivity originate from Pop III BH-BH mergers. Approximately once per decade, we should detect a BH-BH merger that can unambiguously be identified as a Pop III remnant.

On 2017 August 17 the merger of two compact objects with masses consistent with two neutron stars was discovered through gravitational-wave (GW170817), gamma-ray (GRB 170817A), and optical (SSS17a/AT2017gfo) observations. The optical source was associated with the earlytype galaxy NGC 4993 at a distance of just $\sim 40 \mathrm{Mpc}$, consistent with the gravitational-wave measurement, and the merger was localized to be at a projected distance of $\sim 2 \mathrm{kpc}$ away from the galaxy's center (Abbott et al., 2017a,b).

Lipunov et al. (1995) predicted the NS-NS merger at a distance of $\leq 50 \mathrm{Mpc}$ and the possibility of detecting GWs!

This prediction was born by the "Scenario Machine" that describes the evolution of gravimagnetic rotators (Lipunov, 1987; Lipunov, \& Postnov, 1988), and recently commented by Giovannelli (2016).

On August 17, 2017 Multimessenger Astrophysics born! As pioneers of the Multifrequency Astrophysics, we are particularly happy!

Poggiani (2018) published an extensive review about the GW170817 event, in which she discussed also the related multimessenger observations. 


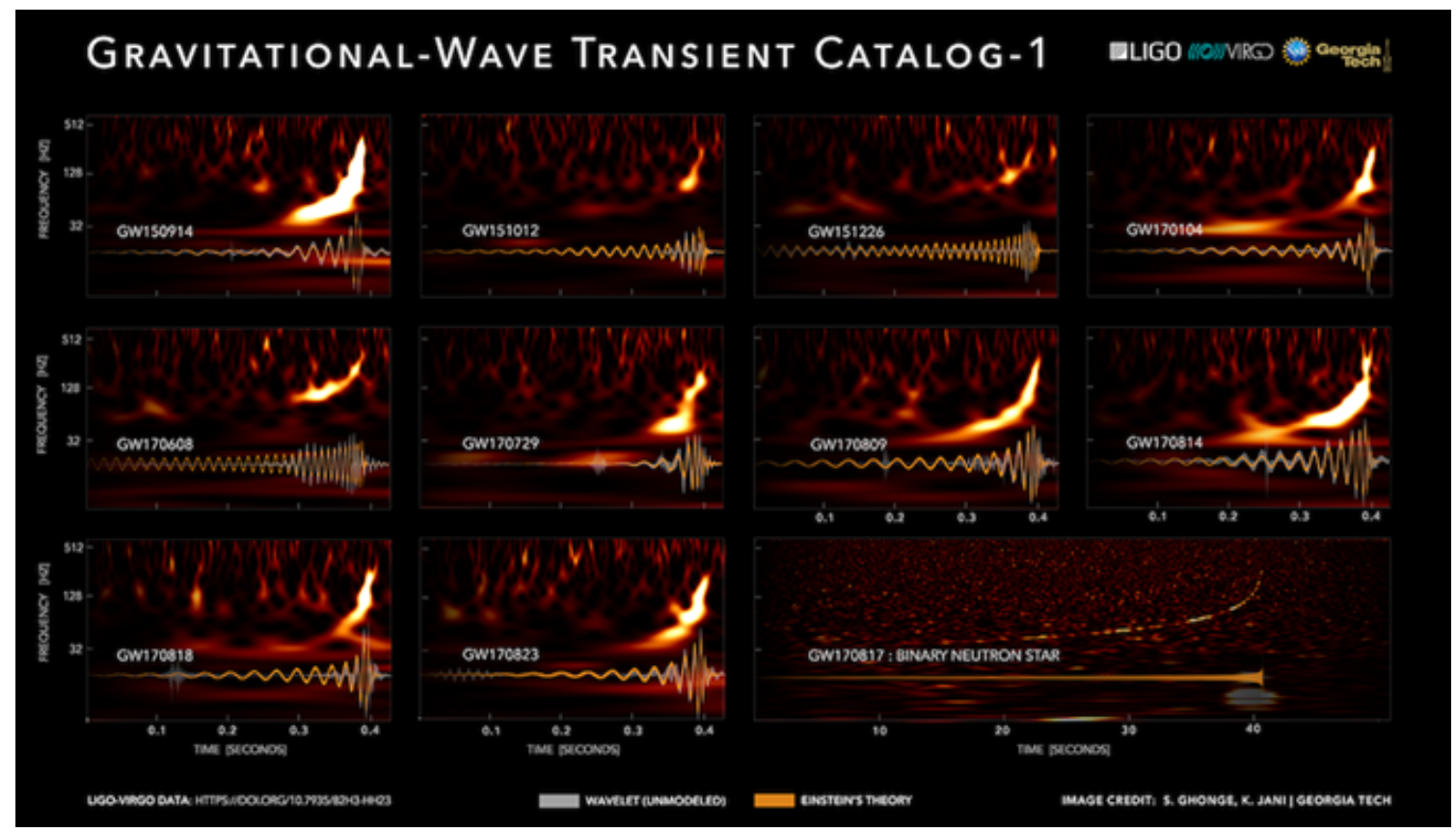

Figure 22: Gravitational Wave Transient Catalog-1. Credit: LIGO Scientific Collaboration and Virgo Collaboration/Georgia Tech/; Image Credit: S. Ghonge \& K. Jani.

The LIGO and Virgo interferometers have now confidently detected gravitational waves from a total of 10 stellar-mass binary black hole mergers and one merger of neutron stars, which are the dense, spherical remains of stellar explosions. Table 1 shows the eleven events (adapted from Abbott et al., 2019).

Figure 22 shows the Gravitational-Wave Transient Catalog-1 where the plots (frequency vs time) of the 11 events listed in Table 1 are reported together with the Einstein's theory predictions (Credit: LIGO Scientific Collaboration and Virgo Collaboration/Georgia Tech/S. Ghonge \& K. Jani).

Figure 23 shows the localizations of the eleven gravitational-wave detections in the sky listed in table 1. The triple detections are labelled as HLV, from the initials of the three interferometers (LIGO-Hanford, LIGO-Livingston and Virgo) that observed the signals. The reduced areas of the triple events demonstrate the capabilities of the global gravitational-wave network (http://www.virgo-gw.eu/ (2018)).

Barone et al. (1992) analyzed the class of CVs as sources of Gravitational Radiation, basing their analysis only on known objects at that time (168 CVs) taken from the Catalog of Ritter (1990).

From the analysis of $\mathrm{GW}$ emission from CVs, they derived that the emission frequencies are in the range $10^{-3}-10^{-5} \mathrm{~Hz}$ and that the GW flux at Earth is in the range $10^{-10}-10^{-13} \mathrm{erg} \mathrm{s}^{-1}$ $\mathrm{cm}^{-2}$ while the dimensionless amplitude is in the range $10^{-21}-10^{-23}$. These results constituted a solid basis for planning the construction of GW detectors (especially space-borne GW antennas). Moreover, these results provided the possibility of experimentally proving the effectiveness of the mechanism of Gravitational Radiation on CV evolution.

This important work was not sufficiently taken into account by the international community. 
Table 1: Selected source parameters of the 11 confident detections. The columns show source-frame component masses $\mathrm{m}_{1}$ and $\mathrm{m}_{2}$, the chirp mass $\mathrm{M}$, final source-frame mass $\mathrm{M}_{f}$, luminosity distance $\mathrm{d}_{L}$, and redshift z (adapted from Abbott et al., 2019).

\begin{tabular}{|c|c|c|c|c|c|c|}
\hline $\begin{array}{l}\text { GW Event } \\
\text { (name) }\end{array}$ & $\begin{array}{l}\mathrm{m}_{1} \\
\left(\mathrm{M}_{\odot}\right)\end{array}$ & $\begin{array}{l}\mathrm{m}_{2} \\
\left(\mathrm{M}_{\odot}\right)\end{array}$ & $\begin{array}{l}\text { M } \\
\left(\mathrm{M}_{\odot}\right)\end{array}$ & $\begin{array}{l}\mathrm{M}_{\mathrm{f}} \\
\left(\mathrm{M}_{\odot}\right)\end{array}$ & $\begin{array}{l}d_{L} \\
(M p c)\end{array}$ & $\begin{array}{l}\mathrm{Z} \\
\text { (redshift) }\end{array}$ \\
\hline GW 150914 & $35.6_{-3.1}^{+4.7}$ & $30.6_{-4.4}^{+3.0}$ & $28.6_{-1.5}^{+1.7}$ & $63.1_{-3.0}^{+3.4}$ & $440_{-170}^{+150}$ & $0.09_{-0.03}^{+0.03}$ \\
\hline GW 151012 & $23.2_{-5.5}^{+14.9}$ & $13.6_{-4.8}^{+4.1}$ & $15.2_{-1.2}^{+2.1}$ & $35.6_{-3.8}^{+10.8}$ & $1080_{-490}^{+550}$ & $0.21_{-0.09}^{+0.09}$ \\
\hline GW 151226 & $13.7_{-3.2}^{+8.8}$ & $7.7_{-2.5}^{+2.2}$ & $8.9_{-0.3}^{+0.3}$ & $20.5_{-1.5}^{+6.4}$ & $450_{-190}^{+180}$ & $0.09_{-0.04}^{+0.04}$ \\
\hline GW 170104 & $30.8_{-5.6}^{+7.3}$ & $20.0_{-4.6}^{+4.9}$ & $21.4_{-1.8}^{+2.2}$ & $48.9_{-4.0}^{+5.1}$ & $990_{-430}^{+440}$ & $0.20_{-0.08}^{+0.08}$ \\
\hline GW 170608 & $11.0_{-1.7}^{+5.5}$ & $7.6_{-2.2}^{+1.4}$ & $7.9_{-0.2}^{+0.2}$ & $17.8_{-0.7}^{+3.4}$ & $320_{-110}^{+120}$ & $0.07_{-0.02}^{+0.02}$ \\
\hline GW 170729 & $50.2_{-10.2}^{+16.2}$ & $34.0_{-10.1}^{+9.1}$ & $35.4_{-4.8}^{+6.5}$ & $79.5_{-10.2}^{+14.7}$ & $2840_{-1360}^{+1400}$ & $0.49_{-0.21}^{+0.19}$ \\
\hline GW 170809 & $35.0_{-5.9}^{+8.3}$ & $23.8_{-5.2}^{+5.1}$ & $24.9_{-2.7}^{+2.1}$ & $56.3_{-3.8}^{+5.2}$ & $1030_{-390}^{+320}$ & $0.20_{-0.07}^{+0.05}$ \\
\hline GW 170814 & $30.6_{-3.0}^{+5.6}$ & $25.2_{-4,0}^{+2,8}$ & $24.1_{-1.1}^{+1.4}$ & $53.2_{-2.4}^{+3.2}$ & $600_{-220}^{+150}$ & $0.12_{-0.04}^{+0.03}$ \\
\hline GW 170817 & $1.46_{-0.10}^{+0.12}$ & $1.27_{-0.09}^{+0.09}$ & $1.186_{-0.001}^{+0.001}$ & $\leq 2.8$ & $40_{-15}^{+7}$ & $0.01_{-0.00}^{+0.00}$ \\
\hline GW 170818 & $35.4_{-4.7}^{+7.5}$ & $26.7_{-5.2}^{+4.3}$ & $26.5_{-1.7}^{+2.1}$ & $59.4_{-3.8}^{+4.9}$ & $1060_{-380}^{+420}$ & $0.21_{-0.07}^{+0.07}$ \\
\hline GW 170823 & $39.5_{-6.7}^{+11.2}$ & $29.0_{-7.8}^{+6.7}$ & $29.2_{-3.6}^{+4.6}$ & $65.4_{-7.4}^{+10.1}$ & $1940_{-900}^{+970}$ & $0.35_{-0.16}^{+0.15}$ \\
\hline
\end{tabular}

However, now, after the detection of GWs coming from the fusion of black holes and neutron stars, the interest for that work has been rekindled in order to test the possibility of detecting GWs from CVs. Poggiani (2017), and the references therein) discussed this possibility, reaching the conclusion that AM CVn systems and generally short-period systems are candidates for GW emission.

Amaro-Seoane et al. (2017) in response to the ESA call for L3 mission concepts, presented the Laser Interferometer Space Antenna (LISA) that since 2030 will allow to observe Gravitational Waves from cosmic sources, then to explore a Universe inaccessible otherwise, a Universe where gravity takes on new and extreme manifestations. They concluded as follows: The groundbreaking discovery of Gravitational Waves by ground-based laser interferometric detectors in 2015 has changed astronomy, by giving us access to the high-frequency regime of Gravitational Wave astronomy. By 2030 our understanding of the Universe will have been dramatically improved by new observations of cosmic sources through the detection of electromagnetic radiation and highfrequency Gravitational Waves. But in the low-frequency Gravitational Wave window, below one Hertz, we expect to observe the heaviest and most distant objects. Using our new sense to 'hear' the Universe with LISA, we will complement our astrophysical knowledge, providing access to a part 


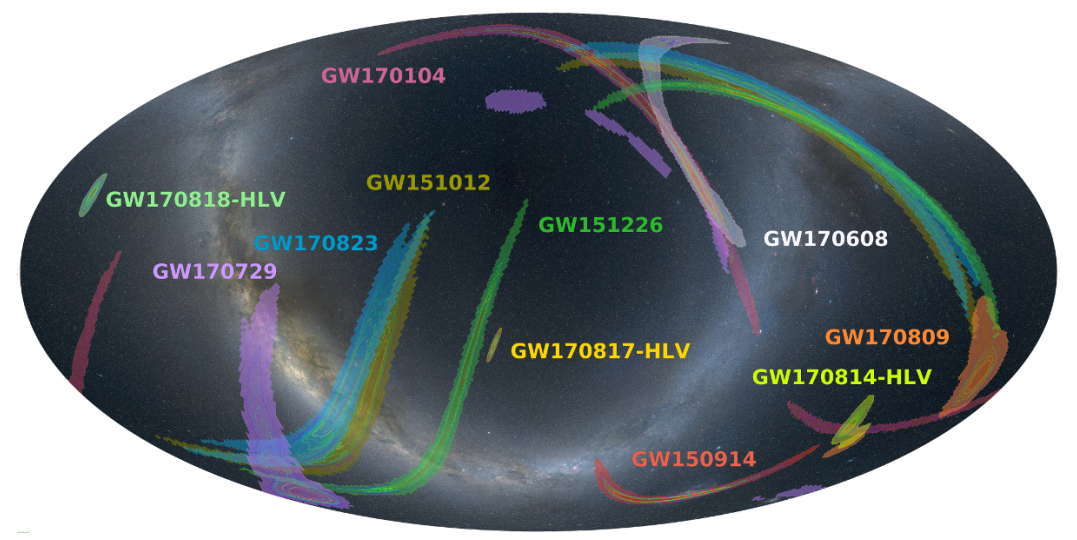

Figure 23: The localizations of the eleven gravitational-wave detections in the sky (http://www.virgo-gw.eu/ (2018)).

of the Universe that will forever remain invisible with light. LISA will be the first ever mission to survey the entire Universe with Gravitational Waves. It will allow us to investigate the formation of binary systems in the Milky Way, detect the guaranteed signals from the verification binaries, study the history of the Universe out to redshifts beyond 20, when the Universe was less than 200 million years old, test gravity in the dynamical sector and strong-field regime with unprecedented precision, and probe the early Universe at TeV energy scales. LISA will play a unique and prominent role in the scientific landscape of the 2030s.

\subsection{The accelerating Universe}

The discovery of the accelerating expansion of the Universe is a milestone for cosmology. A very interesting paper about this argument has been published in 2011 by the "Class for Physics of the Royal Swedish Academy of Sciences" as Scientific Background on the Nobel Prize in Physics 2011. In this paper a historical journey about the last century development of cosmology is brilliantly presented.

The discovery in 1998 that the universe is speeding up and not slowing down (Riess et al. 1998; Perlmutter et al. 1999) opened a question about the possibility of having different phases of acceleration and deceleration of the Universe along its life. Turner \& Riess (2002) from observations of SN 1997ff at $\mathrm{z} \sim 1.7$ favor the accelerating universe interpretation and provide some direct evidence that the universe was once decelerating. They show that the strength of this conclusion depends upon the nature of the dark energy causing the present acceleration. Only for a cosmological constant is the SNe evidence definitive. Using a new test which is independent of the contents of the universe, they show that the SN data favor recent acceleration $(\mathrm{z}<0.5)$ and past deceleration $(\mathrm{z}>0.5)$.

Nielsen, Guffanti \& Sarkar (2016) found marginal evidence for cosmic acceleration from type Ia Supernovae. On the contrary, Haridasu et al. (2017) found that the SN data alone indicate an accelerating Universe at more than $4.56 \sigma$ confidence level.

Considering that some divergent conclusions about cosmic acceleration were obtained using Type Ia supernovae (SNe Ia), with opposite assumptions on the intrinsic luminosity evolution, Tu, Hu \& Wang (2019) use strong gravitational lensing systems to probe the cosmic acceleration. They 
found that the flat $\Lambda \mathrm{CDM}$ is strongly supported by the combination of the data sets from 152 strong gravitational lensing systems.

\subsection{The Big Bang Nucleosynthesis theory has been proved}

The Big Bang Nucleosynthesis (BBN) theory predits the presence of a fixed content of light elements, the temperature of the Universe inversely proportional to the typical distance between galaxy clusters: $\mathrm{T}=\mathrm{T}(0)(1+\mathrm{z})$, and the $\mathrm{CMB}$ radiation temperature of $\sim 2.7 \mathrm{~K}$ (for details see the paper by Giovannelli \& Sabau-Graziati, 2016c).

\subsection{Is the Universe Flat?}

(Schuecker, P., 2005)

(de Bernardis, P. et al., 2000)

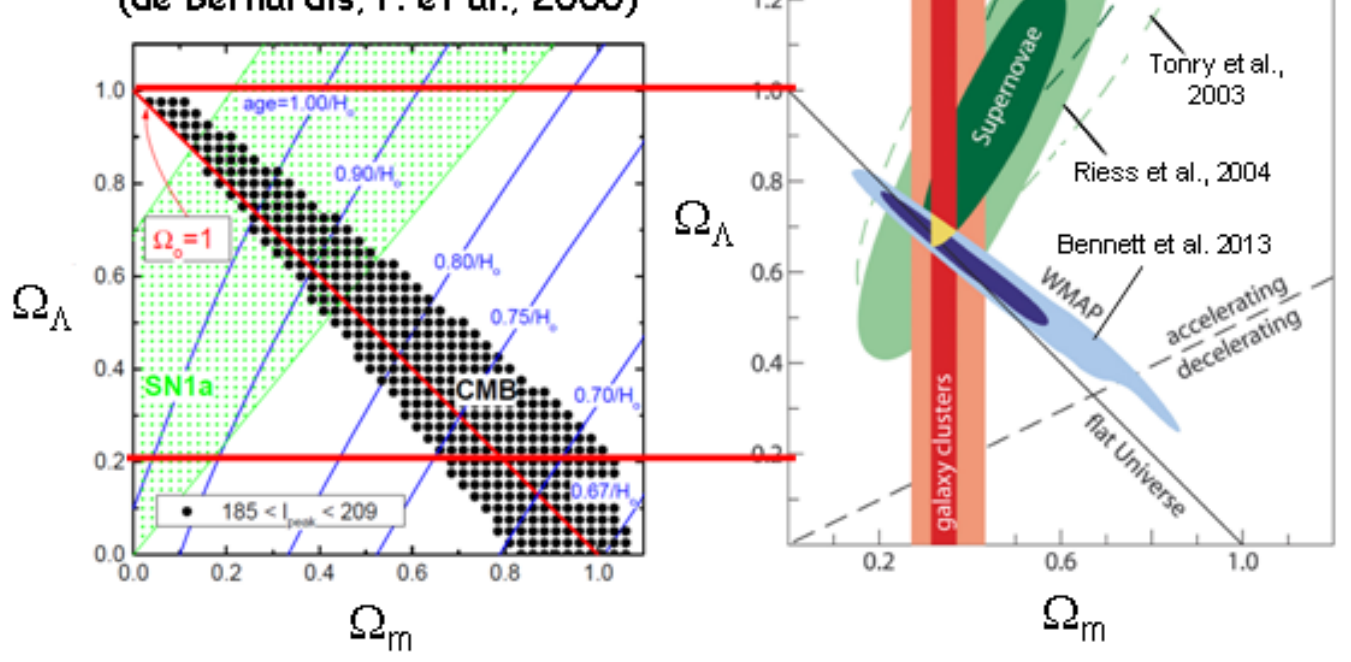

Figure 24: Constraints of cosmological parameters (after de Bernardis et al., 2000; Schuecker, 2005, Bennett et al., 2013).

One of the most critical points about our Universe is the problem of its flatness. The present state of the cosmological tests is illustrated in Fig. 24.

The left panel of Fig. 24 shows the results obtained with the BOOMERanG (Balloon Observations Of Millimetric Extragalactic Radiation and Geomagnetics) experiment (de Bernardis et al., 2000). They are fully consistent with a spatially flat Universe. The right panel of Fig. 24 shows the combination of the likelihood contours obtained with three different observational approaches: i) type-Ia SNe (Tonry et al., 2003; Riess et al. 2004); ii) CMB (Spergel et al. 2003; Bennett et al. 2013); iii) galaxy clusters (Schuecker et al. 2003; Schuecker, 2005). One can see that the cosmic matter density is close to $\Omega_{m}=0.3$, and that the normalized cosmological constant is around $\Omega_{\Lambda}=$ 0.7. This sums up to unit total cosmic energy density and suggests a spatially flat universe. However, the density of cosmic matter growths with redshift like $(1+z)^{3}$ whereas the density $\rho_{\Lambda}$ related to the cosmological constant $\Lambda$ is independent of $z$. The final results from WMAP (Bennett et al., 
2013) show a little misalignment with the line of "flat Universe". Thus it is necessary to be careful in the conclusions.

\subsection{Hubble Constant}

The Hubble constant $\left(\mathrm{H}_{0}\right)$ is one of the most important numbers in cosmology because it is needed to estimate the size and age of the universe. The important problem of determination of $\mathrm{H}_{0}$ value is one of the most exciting. Indeed, in the literature it is possible to find many determinations coming from different experiments using different methods. However, it is very complicated to obtain a true value for $\mathrm{H}_{0}$. It is necessary to have two measurements: i) spectroscopic observations that reveal the galaxy's redshift, indicating its radial velocity; ii) the galaxy's precise distance from Earth (and this is the most difficult value to determine).

A large summary about the methods used for $\mathrm{H}_{0}$ determination, and its derived values can be found in the Proceedings of the Fall 2004 Astronomy 233 Symposium on "Measurements of the Hubble constant" (Damon et al., 2004). In this book, Teymourian (2004), after a comparison of many constraints on the Hubble constant determinations, reports a value $\mathrm{H}_{0}=68 \pm 6 \mathrm{~km} \mathrm{~s}^{-1}$ $\mathrm{Mpc}^{-1}$.

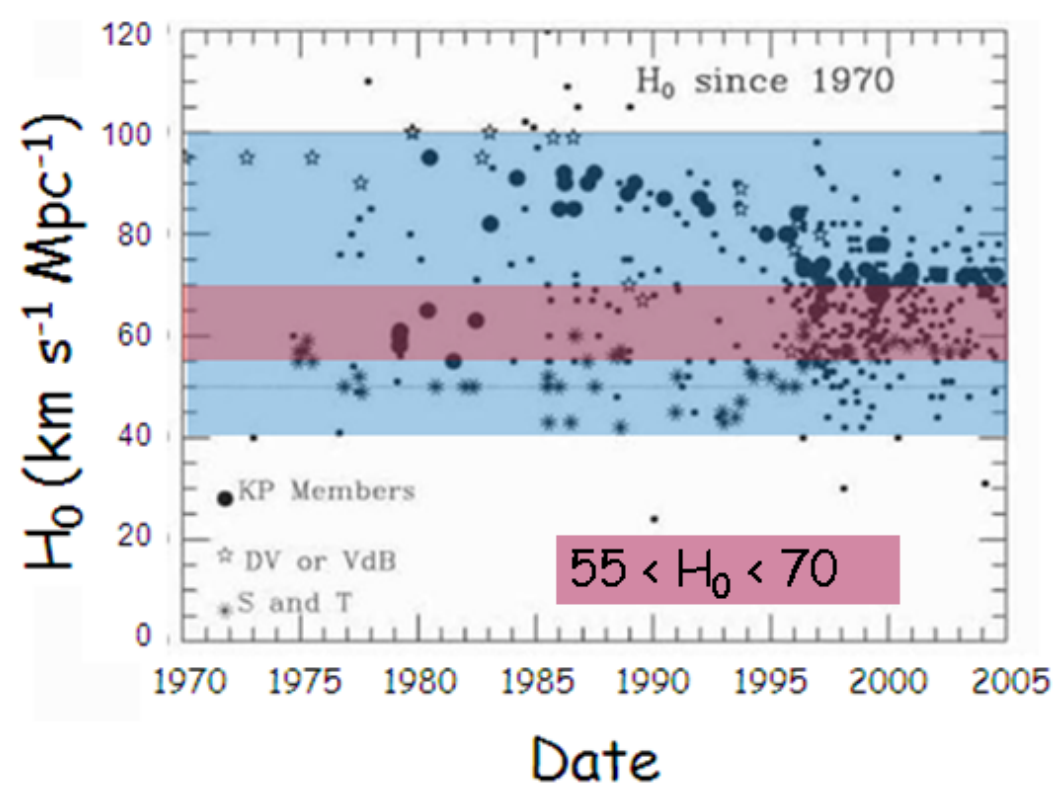

Figure 25: The Hubble constant determinations since 1970. The light-blue rectangle limits all the $\mathrm{H}_{0}$ determinations. The light-red rectangle shows the narrow limits to to which the values of $\mathrm{H}_{0}$ are converging (Giovannelli \& Sabau-Graziati (2016c) after John Huchra, 2008).

Freedman \& Madore (2010) published a review about The Hubble Constant in which they discuss the considerable progress made in determining the Hubble constant over the past two decades. They discuss the cosmological context and importance of an accurate measurement of the Hubble constant, focusing on six high-precision distance-determination methods: Cepheids, tip of the red giant branch, maser galaxies, surface brightness fluctuations, the Tully-Fisher relation, and Type Ia supernovae. Their best current estimate of the Hubble constant is $\mathrm{H}_{0}=73 \pm 2$ (random) \pm 4 (systematic) $\mathrm{km} \mathrm{s}^{-1} \mathrm{Mpc}^{-1}$. 
A discussion about the Hubble constant has been published by Giovannelli \& Sabau-Graziati (2014), where it is possible to find also a large number of references, reporting the many controversial evaluations of $\mathrm{H}_{0}$.

Figure 25 shows the determinations of $\mathrm{H}_{0}$ since 1970 (adapted from John Huchra, 2008). Practically all the determinations lie in the range 40-100 $\mathrm{km} \mathrm{s}^{-1} \mathrm{Mpc}^{-1}$ (marked with light-blue rectangle), and most of them are converging in the range 55-70 $\mathrm{km} \mathrm{s}^{-1} \mathrm{Mpc}^{-1}$ (marked with lightred rectangle).

John Huchra (2010) listed the last updated collection of data on October 7, 2010, just one day before his sudden death (https://www.cfa.harvard.edu/ dfabricant/huchra/hubble.plot.dat). $\left.{ }^{*}\right)$

However, Riess et al. (2011) with the HST determined a value of $\mathrm{H}_{0}=73.8 \pm 2.4 \mathrm{~km} \mathrm{~s}^{-1}$ $\mathrm{Mpc}^{-1}$. This value agrees with the WMAP results: $\mathrm{H}_{0}=71.0 \pm 2.5 \mathrm{~km} \mathrm{~s}^{-1} \mathrm{Mpc}^{-1}$ (Komatsu et al., 2011). Bennett et al. (2014) discussed the progress occurred in recent years for determining the Hubble constant: results coming from the cosmic distance ladder measurements at low redshift and CMB measurements at high redshift.

(*) Professor John Huchra, died unexpectedly October 8th, 2010.

The CMB is used to predict the current expansion rate of the universe by best-fitting cosmological model. At low redshift baryon acoustic oscillation (BAO) measurements have been used - although they cannot independently determine $\mathrm{H}_{0}$ - for constraining possible solutions and checks on cosmic consistency. Comparing these measurements they found $\mathrm{H}_{0}=69.6 \pm 0.7 \mathrm{~km} \mathrm{~s}^{-1} \mathrm{Mpc}^{-1}$.

Does this determination, finally, close the history about the search of the "true" value of $\mathrm{H}_{0}$ ?

However, an important pioneer of science paper (Schutz, 1986) reported how gravitational wave observations can be used to determine the Hubble constant. The nearly monochromatic gravitational waves emitted by the decaying orbit of an ultra-compact, two-neutron-star binary system just before the stars coalesce are very likely to be detected by the kilometer-sized interferometric gravitational wave antennas - at that time being designed. The signal is easily identified and contains enough information to determine the absolute distance to the binary, independently of any assumptions about the masses of the stars. Ten events out to $100 \mathrm{Mpc}$ may suffice to measure the Hubble constant to $3 \%$ accuracy.

Fishbach et al. (2019) performed a statistical standard siren analysis of GW170817. Their analysis did not utilize knowledge of NGC 4993 as the unique host galaxy of the optical counterpart to GW170817. Instead, they consider each galaxy within the GW170817 localization region as a potential host; combining the redshifts from all of the galaxies with the distance estimate from GW170817 provides an estimate of the Hubble constant as $\mathrm{H}_{0}=77_{-18}^{+37} \mathrm{~km} \mathrm{~s}^{-1} \mathrm{Mpc}^{-1}$.

Soares-Santos et al. (2019) presented a multimessenger measurement of the Hubble constant using the binary-black-hole merger GW170814 as a standard siren, combined with a photometric redshift catalog from the Dark Energy Survey (DES). Their analysis results in $\mathrm{H}_{0}=75_{-32}^{+40} \mathrm{~km} \mathrm{~s}^{-1}$ $\mathrm{Mpc}^{-1}$, which is consistent with both SN Ia and CMB measurements of the Hubble constant.

Independent estimation of the Hubble constant from the luminosity distance of GW signal (GW 170817) and the event association with NGC 4993 (Abbott et al., 2017c) gives a value $\mathrm{H}_{0}=$ $70.0_{-8.0}^{+12.0} \mathrm{~km} \mathrm{~s}^{-1} \mathrm{Mpc}^{-1}$. 
However, due to large errors, these values of Hubble constant do not add any significative information, but being obtained with independent methods provide a good support for the value of $\mathrm{H}_{0}=69.6 \pm 0.7 \mathrm{~km} \mathrm{~s}^{-1} \mathrm{Mpc}^{-1}$, determined by Bennett et al. (2014).

\subsection{Reionization Epoch}

Ground-based observations of the CMB on subdegree angular scales suggest that the gas content of the universe was mostly neutral since recombination at $\mathrm{z} \sim 1000$ until about $\mathrm{z} \sim 100$ (Gnedin, 2000) and the references therein) because earlier reionization would have brought the last scattering surface to lower redshift, smoothing the intrinsic CMB anisotropy. At the same time, we know that the universe is highly ionized, since $\mathrm{z} \approx 5$, from observations of the spectra of quasars with the highest redshifts (e.g. Giallongo et al. 1994). This change of the ionization state of the universe from neutral to highly ionized is called "reionization". How large is the redshift to which the reionization started and stopped is object of strong debate.

The formation of the first stars and quasars marks the transformation of the universe from its smooth initial state to its clumpy current state. In current cosmological models, the first sources of light began to form at a redshift $\mathrm{z} \sim 30$ and reionized most of the hydrogen in the universe by $\mathrm{z} \sim 7$ (see review by Loeb \& Barkana, 2001).

Recently Matsuoka et al. (2016) reported the discovery of 15 QSOs and bright galaxies at $5.7<z<6.9$ from the Subaru High-z Exploration of Low-Luminosity Quasars (SHELLQs) project.

The argument for an extended period of reionization is now proved by measurements. Indeed, the WMAP has detected the correlation between temperature and polarization on large angular scales (Kogut et al., 2003) that has an amplitude proportional to the total optical depth of CMB photons to Thomson scattering, $\tau$ (Kaplinghat et al., 2003; Sunyaev \& Zeldovich, 1980; Zaldarriaga, Spergel \& Seljak, 1997).

Modeling reionization with a single sharp transition at $z_{\mathrm{ri}}$, a multi-parameter fit to the WMAP data gives $z_{\mathrm{ri}}=17 \pm 5$ (Spergel et al., 2003). On the other hand, the evolution of quasar spectra from $\mathrm{z} \approx 7$ and $\mathrm{z} \approx 6$ shows a rapid decrease in the amount of neutral hydrogen, indicating the end of reionization (Fan et al., 2003). A simple interpretation to explain these two very different datasets is that reionization started early, $\mathrm{z}_{\mathrm{ri}} \sim 20$, but did not conclude until much later $(\mathrm{z} \sim 6$ ) (Knox, 2003).

This was also confirmed by the results from Subaru Deep Field (SDF) (Kashikawa et al., 2006; Kashikawa, 2007): the reionization of the universe has not been completed at $\mathrm{z}=6.5$. Also Ota et al. (2008) in performing narrowband imaging of the SDF found two Ly $\alpha$ emitters (LAEs) at $\mathrm{z}=$ 7. This established a new redshift record, showing that galaxy formation was in progress just 750 Myr after the Big Bang. They found that the attenuation of the Ly $\alpha$ photons from LAEs by the neutral hydrogen possibly left at the last stage of cosmic reionization at $\mathrm{z} \sim 6-7$.

Ouchi et al. (2009a) suggested an existence of a well-developed ionized bubble at $\mathrm{z}=7$. Ouchi et al. (2009b) reported the discovery of a giant LAE with a Spitzer/Infrared Array Camera (IRAC) counterpart near the reionization epoch at $\mathrm{z}=6.595$. Although the nature of this object is not yet clearly understood, this could be an important object for studying cooling clouds accreting onto a massive halo, or forming-massive galaxies with significant outflows contributing to cosmic reionization and metal enrichment of intergalactic medium. 
Ouchi et al. (2010) presented the Ly $\alpha$ luminosity function (LF), clustering measurements, and Ly $\alpha$ line profiles based on the largest sample to date of 207 LAEs at $\mathrm{z}=6.6$. The combination of various reionization models and their observational results about the LF, clustering, and line profile indicates that there would exist a small decrease of the intergalactic medium's (IGM's) Ly $\alpha$ transmission owing to reionization, but that the hydrogen IGM is not highly neutral at $\mathrm{z}=6.6$. Their neutral-hydrogen fraction constraint implies that the major reionization process took place at $\mathrm{z} \gtrsim 7$.

Jiang et al. (2011) presented Keck spectroscopic observations of $z>6$ Lyman-break galaxy (LBG) candidates in the Subaru Deep Field (SDF). Their Ly $\alpha$ LF is also generally in agreement with the results of LAEs surveys at $\mathrm{z} \sim 5.7$ and 6.6. This study shows that deep spectroscopic observations of LBGs can provide unique constraints on both the UV and Ly $\alpha$ LFs at $\mathrm{z}>6$.

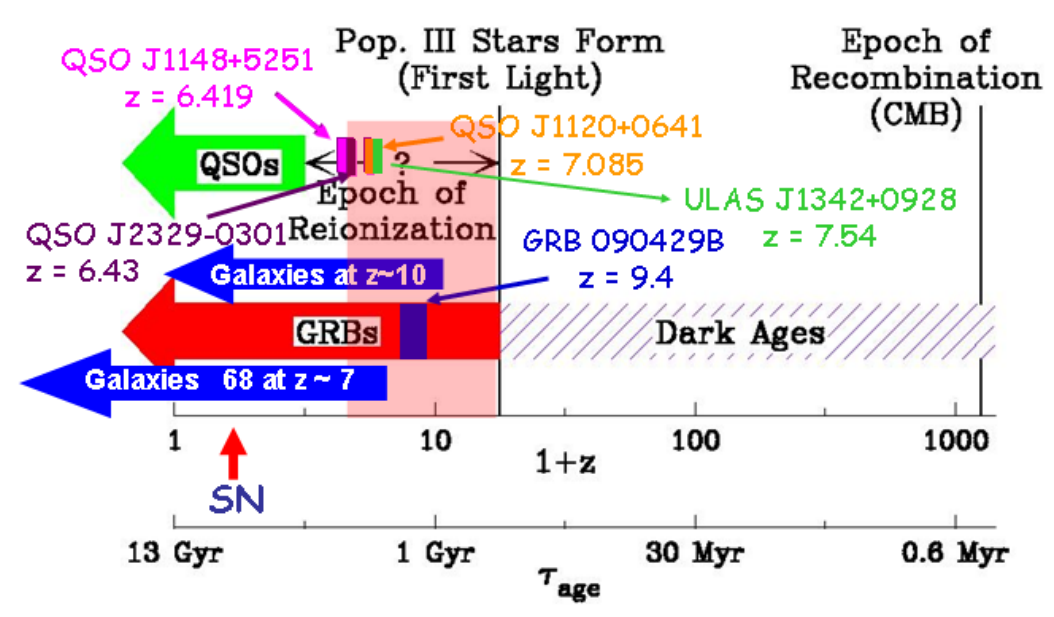

Figure 26: A sketch of reionization epoch (after Xiangping Wu's Talk at the Summer School on "Cosmic Reionization" at the KIAA-PKU , Beijing, China, July 1-11, 2008).

Ono et al. (2012) presented the results of their ultra-deep Keck/DEIMOS spectroscopy of zdropout galaxies in the SDF and Great Observatories Origins Deep Survey's northern field. The fractions of Ly $\alpha$-emitting galaxies drop from $\mathrm{z} \sim 6$ to 7 and the amplitude of the drop is larger for faint galaxies than for bright galaxies. These two pieces of evidence would indicate that the neutral hydrogen fraction of the IGM increases from $\mathrm{z} \sim 6$ to 7 and that the reionization proceeds from high- to low-density environments, as suggested by an inside-out reionization model.

The WMAP detection of reionization (Kogut et al. 2003) implies the existence of an early generation of stars able to reionize the universe at $\mathrm{z} \sim 20$. Panagia et al. (2005) in deep HST/VLT/Spitzer images found that the source UDF 033238.7-274839.8 - a post-starburst galaxy with a mass $\sim 6 \times 10^{11} \mathrm{M}_{\odot}$ placed at $\mathrm{z} \geq 6.5$ - may be capable of reionizing its surrounding region of the universe, starting the process at a redshift as high as $\mathrm{z}=15 \pm 5$.

The question about the end of the reionization is strongly disputed. However, in our opinion probably it is possible to put a reasonable limit to the epoch of the reionization end $(\mathrm{z} \sim 6)$, looking at the paper by Toshikawa et al. (2012). They reported the discovery of a protocluster at $\mathrm{z} \sim 6$ containing at least eight cluster member galaxies with spectroscopic confirmations in the widefield image of the SDF. They found no significant difference in the observed properties, such as 
Ly $\alpha$ luminosities and UV continuum magnitudes, between the eight protocluster members and the seven non-members. The velocity dispersion of the eight protocluster members is $647 \pm 124$ $\mathrm{km} \mathrm{s}^{-1}$, which is about three times higher than that predicted by the standard cold dark matter model. This discrepancy could be attributed to the distinguishing three-dimensional distribution of the eight protocluster members. They discussed two possible explanations for this discrepancy: either the protocluster is already mature, with old galaxies at the center, or it is still immature and composed of three subgroups merging to become a larger cluster. In either case, this concentration of $z=6.01$ galaxies in the SDF may be one of the first sites of formation of a galaxy cluster in the universe.

Figure 26 shows schematically the updated experimental situation about cosmic sources (galaxies, GRBs, QSOs, SNe) detected at high redshifts. The light-red rectangle marks the possible range of $\mathrm{z}$ during which the reionization occurred.

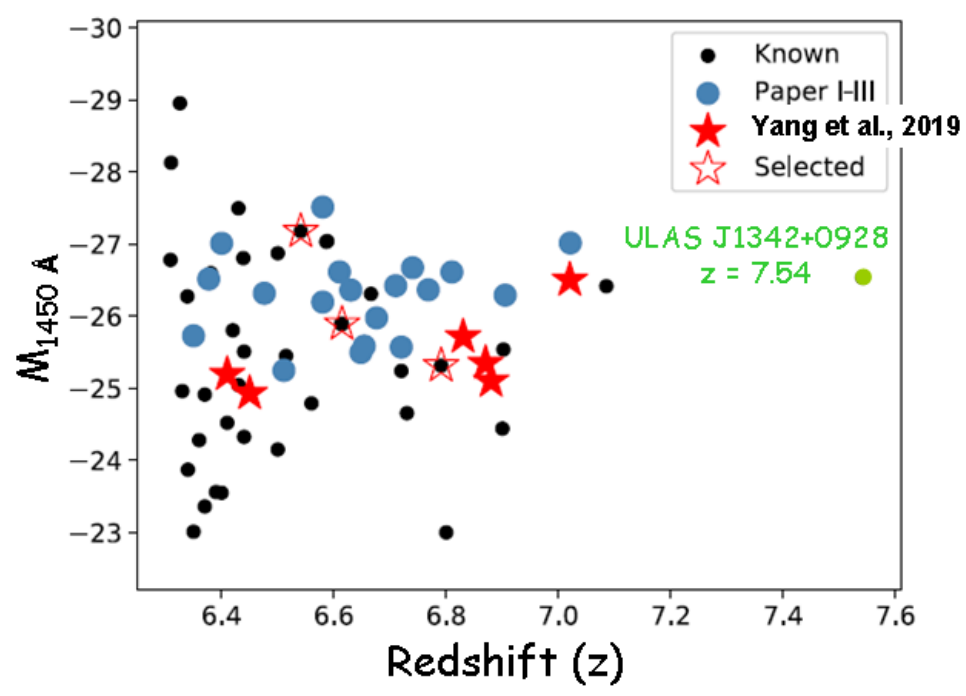

Figure 27: Redshift and $\mathrm{M}_{1450}$ distribution of quasars at $\mathrm{z} \gtrsim 6.3$ (adapted from Yang et al., 2019). The black filled circles are the previously known quasars. The blue filled circles denote quasars from surveys in the northern sky (Wang et al. 2017, 2018, 2019a). The red filled stars represent the six new quasars from Yang et al (2019) survey and the red open stars are the three known quasars in the Dark Energy Survey (DES) area that meet their selection criteria. The green dot represents the $\mathrm{z}=7.54$ quasar ULAS J1342+0928 (Bañados et al., 2019).

However, although there is rather good agreement about the epoch of reionization, how really reionization occurs is still object of debate. Indeed, Dopita et al. (2011), considering that observations show that the measured rates of star formation in the early universe are insufficient to produce reionization, suggest the presence of another source of ionizing photons. This source could be the fast accretion shocks formed around the cores of the most massive haloes.

An interesting review about The epoch of reionization was published by Zaroubi (2013). Recently An Introductory Review on Cosmic Reionization have been published by Wise (2019).

Recently, Yang et al. (2019) announced the discovery of six new z $\gtrsim 6.5$ quasars. They plotted the positions of these new quasars in a diagram magnitude at $1450 \AA$ versus redshift where also the known quasars at $\mathrm{z} \gtrsim 6.3$ have been reported (Fig. 27). The meaning of the symbols are reported 
in the caption of Fig. 27. Note that the $\mathrm{z}=7.54$ quasar ULAS J1342+0928, hosted by a galaxy merger is also reported (green dot) (Bañados et al., 2019).

This work opens a glimmer of light on the possibility of revealing in the future, with the advent of JWST, the presence of quasars immediately after the formation of the first Pop. III stars at $\mathrm{z} \approx$ 25, as well as the possibility of detecting GRBs up to that redshift (Lamb \& Reichart, 2000; Ciardi \& Loeb, 2000; Bromm \& Loeb, 2002). Indeed, the detection of the GRB 090429B at $\mathrm{z} \simeq 9.4$ (Cucchiara et al., 2011) is a good omen to think that future experiments can reveal GRBs up to the fateful threshold of $\mathrm{z} \approx 25$.

\subsection{Star Formation}

In his splendid review, Robert C. Kennicutt, Jr. (1998) discussed the observations of star formation rates (SFRs) in galaxies that provide vital clues to the physical nature of the Hubble sequence and showing that these observations are key probes of the evolutionary histories of galaxies.

About the evolutionary history of galaxies, interesting results were discussed in the review paper "Star-Formation Histories, Abundances, and Kinematics of Dwarf Galaxies in the Local Group" by Tolstoy, Hill \& Tosi (2009). They discussed the results of quantitative studies in nearby dwarf galaxies, since within the Local Universe, galaxies can be studied in great detail star by star. The color-magnitude diagram synthesis method is well established as the most accurate way to determine star-formation histories of galaxies back to the earliest times. These studies have shown how the properties of stellar populations can vary spatially and temporally. This leads to important constraints to theories of galaxy formation and evolution. The continuity of structural properties from dwarf galaxies to larger spheroidal and late-type systems is most likely dominated by physical processes that scale with mass, for example, the efficiency with which gas and/or metals can be lost from a system during its evolution through supernova winds and/or interactions.

Zinnecker \& Yorke (2007) discussed in their review a basic description of the collapse of a massive molecular core and a critical discussion of the three competing concepts of massive star formation:

- monolithic collapse in isolated cores;

- competitive accretion in a protocluster environment;

- stellar collisions and mergers in very dense systems.

They concluded that high-mass star formation is not merely a scaled-up version of low-mass star formation with higher accretion rates, but partly a mechanism of its own, primarily owing to the role of stellar mass and radiation pressure in controlling the dynamics.

Kennicutt, Jr \& Evans II (2012) reviewed the progress over the previous decade in observations of large-scale star formation, with a focus on the interface between extragalactic and galactic studies. Methods of measuring gas contents and star-formation rates have been discussed, and updated prescriptions for calculating star-formation rates were provided. They reviewed relations between star formation and gas on scales ranging from entire galaxies to individual molecular clouds.

The key dynamical processes involved in star formation - turbulence, magnetic fields, and self-gravity - are highly nonlinear and multidimensional. Therefore, it is extremely difficult a 

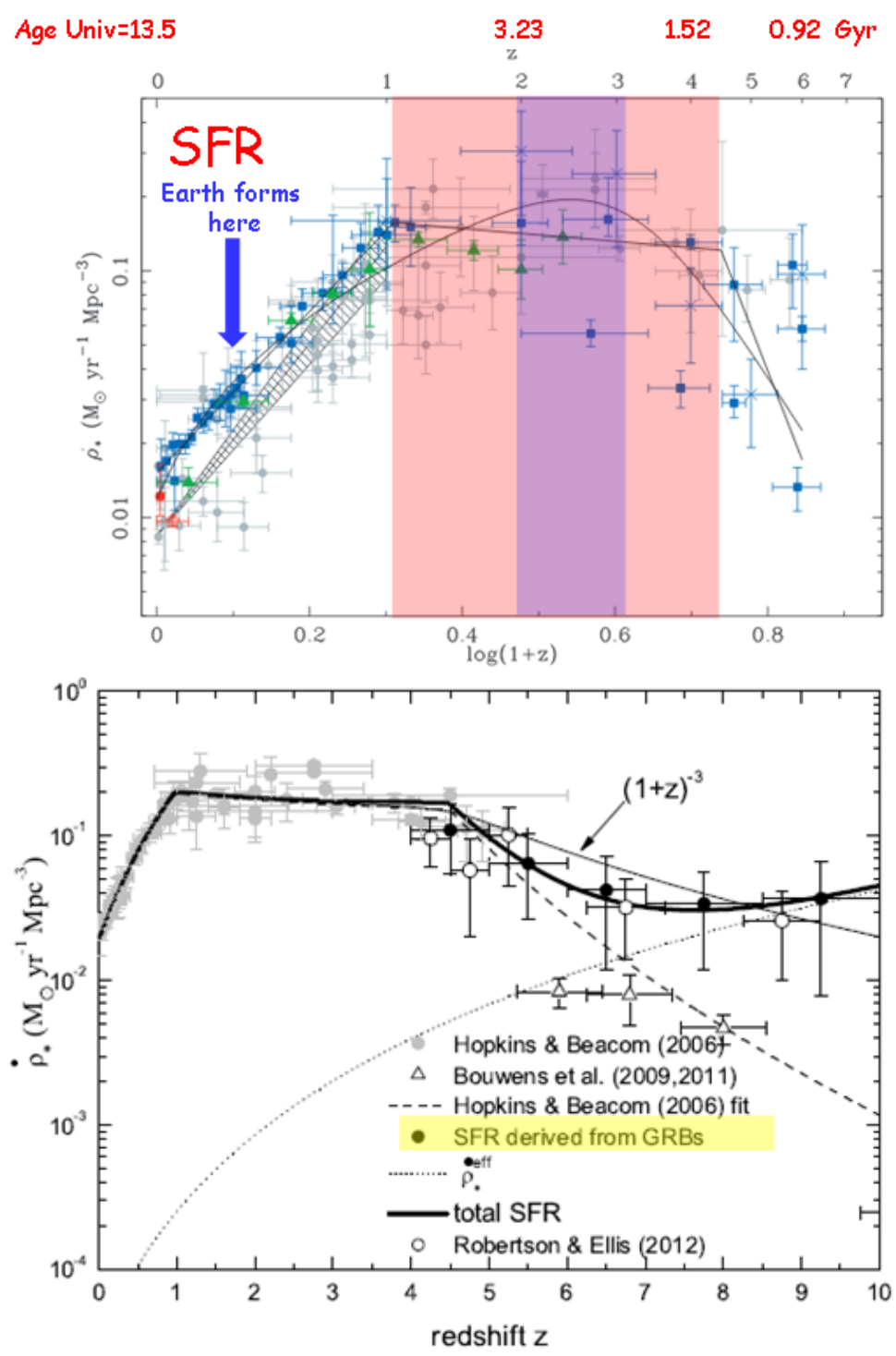

Figure 28: Upper panel: Evolution of SFR density with redshift (after Madau \& Dickinson, 2014). Lower panel: Evolution of SFR density with redshift; black dots for $\mathrm{z} \gtrsim 4.5$ mark the values of SFR density derived from GRBs (Wang, Dai \& Liang, 2015).

complete quantitative description of the physics involved in the process of star formation. McKee \& Ostriker (2007) attempted to review the theory of star formation. For this reason they divided star formation into large-scale and small-scale regimes and reviewed each in turn. Large scales range from galaxies to giant molecular clouds (GMCs) and their substructures. Important problems include how GMCs form and evolve, what determines the star formation rate (SFR), and what determines the initial mass function (IMF) have been discussed. Small scales range from dense cores to the protostellar systems they beget. They discussed formation of both low- and high-mass stars, including ongoing accretion. The development of winds and outflows is increasingly well understood, as are the mechanisms governing angular momentum transport in disks. However, they concluded that a comprehensive theory of star formation will be tested by the next generation 
of telescopes.

Fumagalli et al. (2012) investigated the evolution of the $\mathrm{H}_{\alpha}$ equivalent width, $\mathrm{EW}\left(\mathrm{H}_{\alpha}\right)$, with redshift and its dependence on stellar mass, using the first data from the 3D-HST survey, a large spectroscopic Treasury program with the HST-WFC3. Combining these data with those from ground-based telescopes, they found that at all masses the characteristic $\operatorname{EW}\left(\mathrm{H}_{\alpha}\right)$ is decreasing towards the present epoch, and that at each redshift the $\mathrm{EW}\left(\mathrm{H}_{\alpha}\right)$ is lower for high-mass galaxies.

The cosmic history of star formation, heavy element production, and reionization of the Universe from the cosmic "dark ages" to the present epoch has been discussed in the review paper by Madau \& Dickinson (2014). A consistent picture is emerging, whereby the star-formation rate density peaked approximately 3.5 Gyr after the Big Bang, at $\mathrm{z} \approx 1.9$, and declined exponentially at later times, with an e-folding timescale of 3.9 Gyr. Half of the stellar mass observed today was formed before a redshift $\mathrm{z}=1.3$. About $25 \%$ formed before the peak of the cosmic star-formation rate density, and another $25 \%$ formed after $\mathrm{z}=0.7$. Less than $\sim 1 \%$ of today's stars formed during the epoch of reionization.

However, these results were already largely discussed and presented by Hopkins \& Beacom (2006), and later by Madau \& Dickinson (2014) and summarized in the Fig. 28 (upper panel). The light-red rectangle marks the range of redshift where the star formation density had the maximum $(1 \lesssim \mathrm{z} \lesssim 4.5$ whose peak is between $\mathrm{z}=2$ and $\mathrm{z}=3$ and marked with the light violet rectangle. This will be better understood when the supernova rate density evolution, the ranges of stellar masses leading to core-collapse and type Ia supernovae, and the antineutrino and neutrino backgrounds from core-collapse supernovae will be known thanks to the next generation experiments both ground- and space-based. Figure 28 (lower panel) clearly show the values of SFR (black dots) derived from GRBs by Wang, Dai, \& Liang (2015) at redshifts $\mathrm{z} \gtrsim 4.5$.

A recent book about "Star Formation in Galaxy Evolution: Connecting Numerical Models to Reality" (Gnedin et al., 2015) reports an inventory of the physical processes related to the star formation involved at different scales and also to provide an overview of the major computational techniques used to solve the equations governing self-gravitating fluids, essential to galactic modeling. Together this provides a unique framework essential to developing and improving the simulation techniques used to understand the formation and evolution of galaxies.

\subsection{Extragalactic Background Light}

The intergalactic space is filled with the light produced by all the stars and accreting compact objects that populated the observable Universe throughout the whole cosmic history. This relic cosmic background from IR to UV is called the diffuse Extragalactic Background Light (EBL), long before known as DEBRA (Ressel \& Turner, 1990).

Direct measurements of the EBL are difficult due to bright local foregrounds. A powerful approach for probing these diffuse radiation fields in the UV to far-IR bands is through $\gamma-\gamma$ absorption of high-energy photons. Actually pair production $\left(\mathrm{e}^{+} \mathrm{e}^{-}\right)$against EBL photons with wavelengths from ultraviolet to infrared is effective at attenuating $\gamma$-rays with energy above $\sim 10 \mathrm{GeV}$. This process introduces an attenuation in the spectra of $\gamma$-ray sources above a critical energy (e.g. Costamante, 2012; Buson, 2014).

The last decade has been foreboding of a full coverage of the HE-VHE $\gamma$-ray energy band, thanks to the many ground- and space-based high sensitivity experiments. Thus it has been possi- 


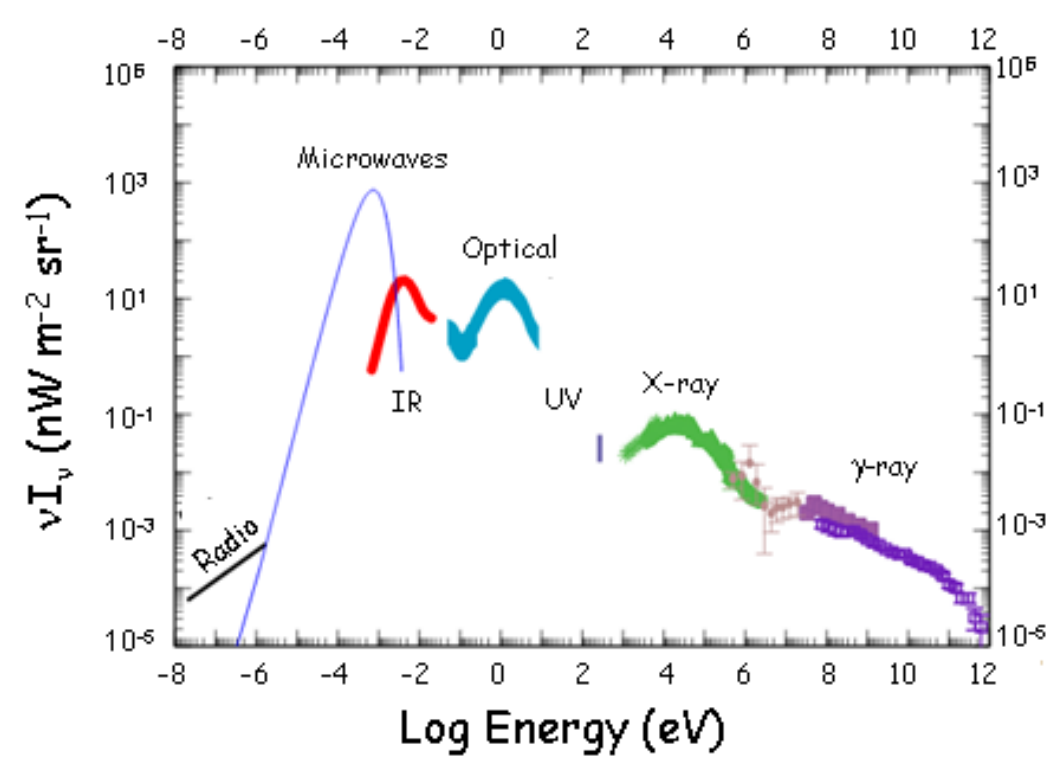

Figure 29: Intensity of the extragalactic background $\left(v \mathrm{I}_{v}\right.$ in units of $\left.\mathrm{nW} \mathrm{m} \mathrm{m}^{-2} \mathrm{sr}^{-1}\right)$ as a function of the energy (after Cooray, 2016).

ble to collect a large amount of data from many extragalactic emitters at high redshift (e.g. Costamante, 2012). Thanks to measurements of the quasar 3C 279 ( $\mathrm{z} \simeq 0.54$ ) obtained with the MAGIC experiment (Albert et al., 2008), and with the many sources at high redshift, including Gamma Ray Bursts (GRBs) measured with the FERMI observatory (Abdo et al., 2010), it has been demonstrated that the Universe is more transparent to $\gamma$-rays than before believed (Coppi \& Aharonian, 1997).

Cooray (2016) reviews the Extragalactic Background Light Measurements and Applications. This review covers the measurements related to the extragalactic background light intensity from $\gamma$-rays to radio in the electromagnetic spectrum over 20 decades in wavelength. Figure 29 shows such EBL measurements that updated those reported by Ressel \& Turner (1990). It is important to remark that the numerous measurements in the range of the VHE $\gamma$-rays $(\log \mathrm{E} \approx 9-13 \mathrm{eV})$ have filled the zone where no measurements or only upper limits were available in the 1990-ies.

The CMB remains the best measured spectrum with an accuracy better than $1 \%$. Durrer (2015) in her interesting review describes the discovery of the cosmic microwave background radiation in 1965 and its impact on cosmology in the 50 years that followed.

Henry et al. (2015) discussed the diffuse cosmic background radiation in the Galaxy Evolution Explorer far-ultraviolet (FUV, 1300-1700 A. They deduced that the UV diffuse cosmic background radiation originates only partially in the dust-scattered radiation of FUV-emitting stars: the source of a substantial fraction of the FUV background radiation remains a mystery. They also discussed about our limited knowledge of the cosmic diffuse background at ultraviolet wavelengths shortward of Ly $\alpha$ - it could be that a "second component" of the diffuse FUV background persists shortward of the Lyman limit and is the cause of the reionization of the universe. 


\subsection{Gamma Ray Bursts}

Long discussions about Gamma-ray bursts (GRBs) can be found in numerous publications. A list of these can be found in GSG2004 and in Giovannelli \& Sabau-Graziati (2016c).

Although big progress has been obtained in the last few years, GRBs theory needs further investigation in the light of the experimental data coming from old and new satellites, often coordinated, such as BeppoSAX or BATSE/RXTE or ASM/RXTE or IPN or HETE or INTEGRAL or SWIFT or AGILE or FERMI or MAXI. Indeed, in spite of thousands papers appeared in the literature since the discovery of GRBs, the problem of their energy emission is still elusive: i) what is jet's composition? (kinetic or magnetic?); ii) where is dissipation occurring? (photosphere? deceleration radius?); iii) how is radiation generated? (synchrotron, Inverse Compton, hadronic?) (Zhang, 2013a,b).

Kumar \& Zhang (2015) in a review paper The Physics of Gamma-Ray Bursts \& Relativistic Jets discussed what we have learned about relativistic collisionless shocks and particle acceleration from GRB afterglow studies, and the current understanding of radiation mechanism during the prompt emission phase. They pointed out how these explosions may be used to study cosmology, e.g. star formation, metal enrichment, reionization history, as well as the formation of first stars and galaxies in the Universe.

The idea that GRBs could be associated to gravitational waves (GWs) emission is now popular. Indeed, short GRBs are believed to be produced by the mergers of either double NSs or NS-BH binaries (Nakar, 2007) and the observation of a kilonova associated with GRB130603B (Tanvir et al., 2013; Berger, Fong \& Chornock, 2013) lends support to this hypothesis. In a recent review, D'Avanzo (2015) discussed the observational properties of short GRBs and showed how the study of these properties can be used as a tool to unveil their elusive progenitors and provide information on the nature of the central engine powering the observed emission. The increasing evidence for compact object binary progenitors makes short GRBs one of the most promising sources of gravitational waves for the Advanced LIGO/Virgo experiments. This idea obtained recently its experimental verification with the detection of GW 170817 event associated with the GRB 170817A (Abbott et al,, 2017a,b).

Thanks to the NASA's Swift satellite we assisted to ten years of amazing discoveries in time domain astronomy. Its primary mission is to chase GRBs. The list of major discoveries in GRBs and other transients includes the long-lived X-ray afterglows and flares from GRBs, the first accurate localization of short GRBs, the discovery of GRBs at high redshift $(\mathrm{z}>8)$ (Gehrels \& Cannizzo, 2015). And essentially thanks to these discoveries we are now closer to understand the real nature of GRBs.

The review by Bernardini (2015) discussed how the newly-born millisecond magnetars can compete with black holes as source of the GRB power, mainly with their rotational energy reservoir. They may be formed both in the core-collapse of massive stars, and in the merger of neutron star or white dwarf binaries, or in the accretion-induced collapse of a white dwarf, being thus a plausible progenitor for long and short GRBs, respectively.

Ghirlanda et al. (2015) discussed about the apparent separation of short and long GRBs in the hardness ratio vs duration plot. This separation has been considered as a direct evidence of the difference between these two populations. The origin of this diversity, however, has been only 
confirmed with larger GRB samples but not fully understood. They concluded that short and long GRBs have similar luminosities and different energetics (i.e. proportional to the ratio of their average durations). Then, it seems that the results are pointing toward the possibility that short and long GRBs could be produced by different progenitors but the emission mechanism responsible for their prompt emission might be similar.
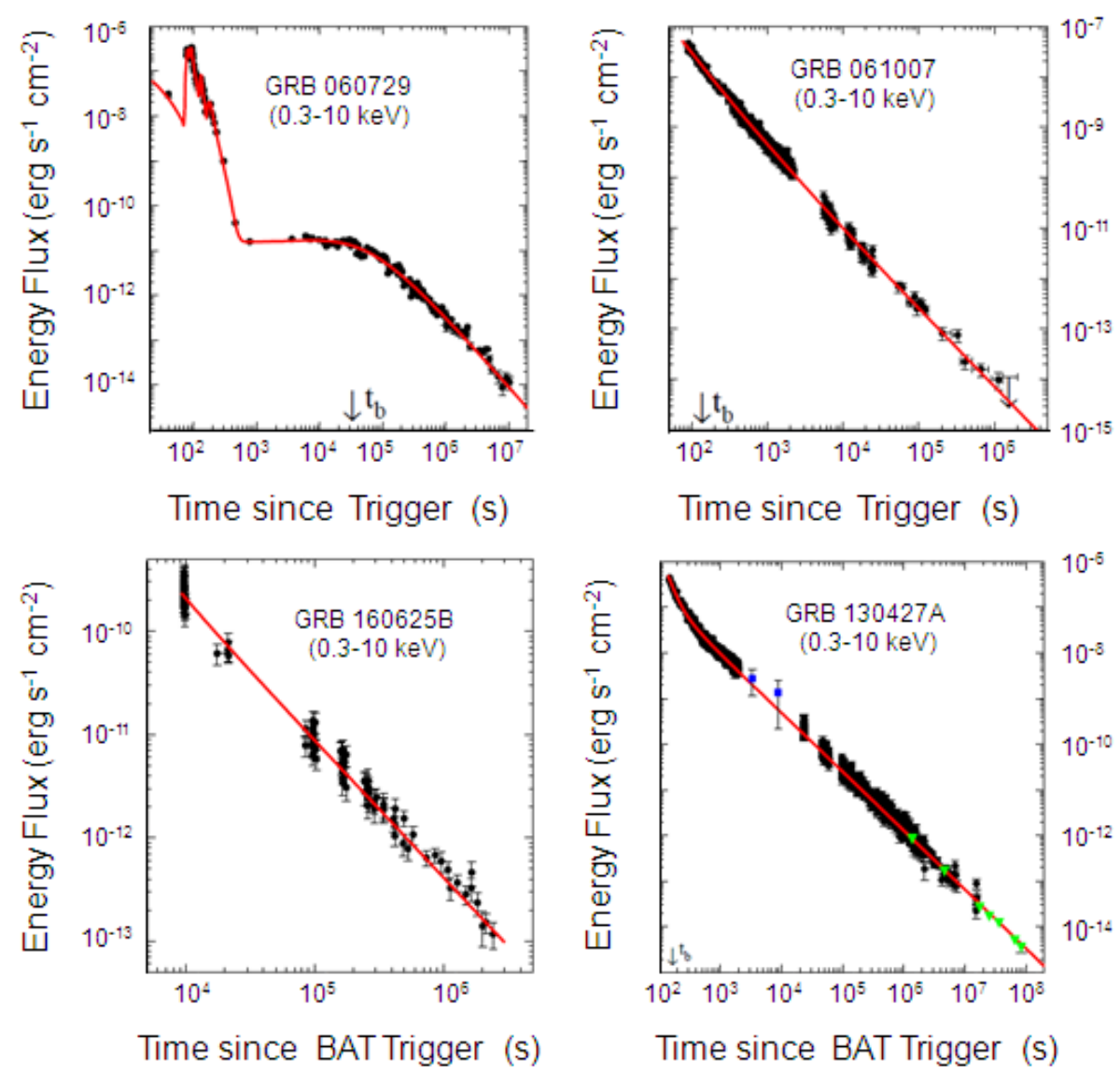

Figure 30: The 0.3-10keV X-ray light-curve measured with the Swift XRT (Evans et al., 2009), and the comparison between Swift observations and their CB-model description (adapted from Dado \& Dar, 2016) for: top left GRB 060729, top right GRB 061007, bottom left GRB 160625B, bottom right GRB 130427A. This latter figure reports data from different experiments: Swift XRT (black circles), XMM Newton and Chandra (green triangles) (De Pasquale et al., 2016), and the two MAXI data points (blue squares) (Maselli et al., 2014) at $\mathrm{t}=3257 \mathrm{~s}$ and $\mathrm{t}=8821 \mathrm{~s}$. The BAT trigger time is marked with $\mathrm{t}_{\mathrm{b}}$.

Piron (2016) in his review discussed the updated knowledge of GRBs at very high energies. Their huge luminosities involve the presence of a newborn stellar-mass black hole emitting a relativistic collimated outflow, which accelerates particles and produces non-thermal emissions from the radio domain to the highest energies. He reviewed recent progresses in the understanding of GRB jet physics above $100 \mathrm{MeV}$, based on Fermi observations of bright GRBs, and discussed the physical implications of these observations and their impact on GRB modeling.

Recently Arnon Dar (2017) proposed again to the attention of the international community his Cannonball (CB) model for explaining the physics of GRBs. In the CB model, GRBs and their 
afterglows are produced by the interaction of bipolar jets of highly relativistic plasmoids (CBs) of ordinary matter with the radiation and matter along their trajectory. Such jetted CBs are presumably ejected in accretion episodes of fall-back material on the newly formed compact stellar object in core-collapse supernovae ( $\mathrm{SNe}$ ) of Type Ic, in merger of compact stellar objects in close binary systems, and in phase transitions in compact stars (Shaviv \& Dar, 1995; Dar, 1997; Dar \& De Rujula, 2000; Dado \& Dar, 2013a). Dado, Dar \& De Rújula (2009) discussed a long series of different SWIFT GRBs, showing that the CB model fits all their broadband light curves. Dado \& Dar (2013b) discussed the jet break in the X-ray afterglow of GRBs that appears to be correlated to other properties of the X-ray afterglow and the prompt gamma ray emission, but the correlations are at odds with those predicted by the conical fireball (FB) model of GRBs (Piran, 1999). On the contrary they are in good agreement, however, with those predicted by the CB model of GRBs.

Finally, Dado \& Dar (2016) discussed on the critical test of gamma-ray bursts theories and demonstrated definitively the validity of the CB model against the popular FB model (Piran, 1999).

Figure 30 shows, as example, the fits of light curves of GRB 060729, GRB 061007, GRB 160625B, and GRB 130427A by using the CB model.

In our opinion the problem of the models for explaining the behaviour of GRBs can be considered closed. The CB model is the best in absolute for the description of the physics governing the GRBs.

However, recent papers have been published about statistical studies of GRBs.

Zhao et al. (2019) performed an analysis for the shallow decay component of GRBs X-ray afterglow, in order to explore its physical origin with an updated sample of GRBs - data from Swift/XRT GRBs between February 2004 and July 2017 - with respect to that used by Liang et al. (2007) based on the early-year observations from Neil Gehrels Swift Observatory.

Overall, their results are generally consistent with Liang, Zhang \& Zhang (2007), confirming their suggestion that the shallow decay segment in most bursts is consistent with an external forward shock origin, probably due to a continuous energy injection from a long-lived central engine.

Tang et al. (2019) considering that a plateau phase in the X-ray afterglow is observed in a significant fraction of GRBs performed a statistical study of this class. Previously, it has been found that there exists a correlation among three key parameters concerning the plateau phase, i.e., the end time of the plateau phase in the GRB rest frame $\left(T_{a}\right)$, the corresponding X-ray luminosity at the end time $\left(\mathrm{L}_{\mathrm{X}}\right)$ and the isotropic energy of the prompt GRB $\left(\mathrm{E}_{\gamma, \text { iso }}\right)$. They systematically searched through all the Swift GRBs with a plateau phase that occurred between 2005 May and 2018 August. They collected 174 GRBs, with redshifts available for all of them. They confirmed that a correlation exist and the best fit gives $\mathrm{L}_{\mathrm{X}} \propto \mathrm{T}_{\mathrm{a}}^{-1.01} \mathrm{E}_{\gamma, \text { iso }}^{0.84}$. Such an updated three-parameter correlation still supports that the central leftover after GRBs is probably a millisecond magnetar. It is interesting to note that short GRBs with duration less than $2 \mathrm{~s}$ in their sample also follow the same correlation, which hints that the merger production of two neutron stars could be a high mass magnetar, but not necessarily a black hole. Moreover, GRBs having an "internal" plateau (i.e., with a following decay index being generally smaller than -3) also obey this correlation. It further strengthens the idea that the internal plateau is due to the delayed collapse of a high mass neutron star into a black hole. The updated three-parameter correlation indicates that GRBs with a plateau phase may act as a standard candle for cosmology study. 
Wang et al. (2019b) performed a comprehensive statistical study using 6289 GRBs. They arrived to the conclusion that in order to reveal more physical principles one should try to classify the GRBs into more precise subgroups based on their physical origin, and the classification itself is a process to reveal the intrinsic properties of GRBs. With the detailed classifications, the correlations inside each group may be more tighter and more physical. The correlations are then can be used to study the radiation mechanism as well as the high energy radiation, to be indicators as standard candle or pseudo redshift, and to study the gravitational waves of compact binary mergers.

Thus also after this interesting paper, the answer to the real processes occurring in GRBs is still open.

\subsection{Anomalous X-ray Pulsars and Soft Gamma Repeaters: Magnetars}

Since their discovery, neutron stars (NSs) have excited a broad range of interests not only in the astrophysical context, but also in terms of fundamental physics.

NSs are characterized by extreme conditions, such as dense matter, rapid rotation, and high magnetic field, they have proved to be ideal laboratories to test fundamental physics, which cannot be achieved by ground-based experiments.

Multi-wavelength observations from radio to the highest energy gamma-rays have revealed a remarkable diversity of NSs (Kaspi, 2010).

In the last two decades a new class of X-ray binaries has been recognized. They are X-ray pulsars with properties clearly different from those of the common HMXBs. This new group of pulsars constitutes a subclass of the LMXBs, characterized by lower luminosities, higher magnetic fields and smaller ages than non-pulsating LMXBs. These objects have been called Anomalous Xray Pulsars (AXPs) (e.g. GSG2004, and the references therein) and this is now the current accepted name. Soon after their discovery, this new class of objects, whose nature was recognized to be that of neutron stars, were characterized by a spin periods ranging between $5.5-11.8 \mathrm{~s}-$ and $\dot{\mathrm{P}}$, in the range $0.05-10 \times 10^{-11} \mathrm{~s} \mathrm{~s}^{-1}-$ contrary to the larger spread of those of HMXBs $\left(0.069-\right.$ few $\times 10^{3}$ s). Spin periods of AXPs are monotonically increasing on timescales of $\sim 10^{4}-4 \times 10^{5} \mathrm{yr}$.

Sources we call now soft gamma-ray repeaters (SGRs) were initially confused with GRBs. The history of this misunderstanding is clearly and exhaustively reported in the paper by Hurley (2008). SGRs are transient very short ( $<1 \mathrm{~s}$ ) events, characterized by relatively soft bursts, peaked at $\sim 20-30 \mathrm{keV}$, with super-Eddington luminosity.

The durations and spectra of SGRs are very different from those of GRBs, like clearly shown in Figure 31 (Hurley, 2007).

Measurements of the spin down rates of SGRs and AXPs have been interpreted as evidence of very strong magnetic fields at the collapsed object poles, roughly two orders of magnitude greater than those of the 'normal' $\mathrm{X}$-ray pulsars. For this reason they are now known as 'magnetars'. Their derived magnetic field intensity is $\sim 10^{14}-10^{15} \mathrm{G}$.

The problem of the nature of magnetars is one of the hottest in modern astrophysics. Indeed, for instance, Dar (2003) argued that, instead, the observations support the hypothesis that SGRs and AXPs are neutron stars that have suffered a transition into a denser form of nuclear matter to become, presumably, strange stars or quark stars. Internal heat and slow gravitational contraction long after this transition can power both their quiescent X-ray emission and their star quakes, which produce 'soft' gamma ray bursts. Dar (2006) discussed once more this idea by using results 


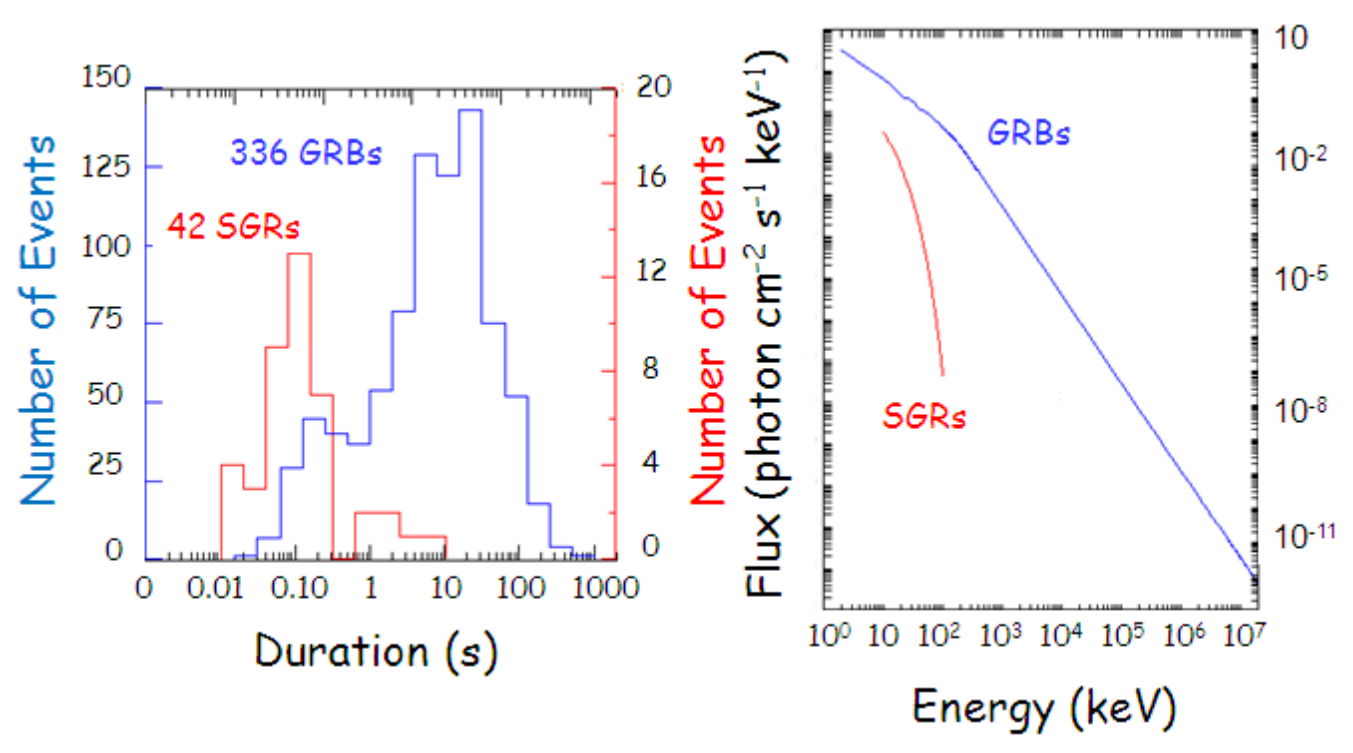

Figure 31: Comparison of the durations and spectra of SGRs and GRBs (adapted from Hurley, 2007).

from short-duration hard-spectrum GRBs, such as 050509B, 050709, 050724, and 050813, which could have been the narrowly beamed initial spike of hyperflares of SGRs in galaxies at cosmological distances. Such bursts are expected if SGRs are young hyperstars, i.e. neutron stars where a considerable fraction of their neutrons have converted to hyperons and/or strange quark matter. Ghosh (2009) discussed some of the developments in the quark star physics along with the consequences of possible hadron to quark phase transition at high density scenario of neutron stars and their implications on the Astroparticle Physics.

Models based on strongly magnetized neutrons stars $\left(B \sim 10^{14}-10^{15} \mathrm{G}\right)$ have been developed in the last couple decades just in order to explain the behaviour of the SGRs (Duncan \& Thompson, 1992; Thompson \& Duncan, 1995, 1996). The possibility of having strong magnetic fields is well accepted. Indeed, a neutron star undergoes vigorous convection in the first $\sim 30 \mathrm{~s}$ after its formation. Coupled with rapid rotation ( $\sim 1 \mathrm{~ms}$ period), this makes the neutron star a likely site for dynamo action. If the Rossby number (=rotation period/convective overturn time) $<1$, the magnetic field amplification is not suppressed. In principle a magnetic field of $\mathrm{B} \sim 3 \times 10^{17} \mathrm{G}$ can be generated.

Thus, AXPs and SGRs are interpreted as magnetars, whose definition is a neutron star in which the magnetic field, rather than rotation, provides the main source of free energy; the decaying field powers the electromagnetic radiation (Hurley, 2008 and the references therein). Note that the definition does not specify any particular field strength, but rather, is based on an energy balance argument. Today we know of several possible manifestations of magnetars, and SGRs are one. With inferred magnetic field strengths $\mathrm{B} \sim 10^{15}$ Gauss, magnetars indeed have the strongest cosmic magnetic fields that we know of in the Universe. But we also know of neutron stars with strong magnetic fields that are rotation-powered, and clearly do not fit the magnetar description (e.g. McLaughlin et al. 2003).

For long time the optical counterparts of AXPs were not known, with the possible exception 
of 4U 0142+61, for which Hulleman, van Kerkwijk \& Kulkarni (2000) reported the discovery of a faint ( $R \sim 25 \mathrm{mag}$ ) blue object in its error box. If such an association should be true, this faintness would rule out the presence of an accretion disk, thus favoring the magnetar interpretation. On the basis of the limits in the optical and IR wavelength regions, the presence of a massive early type companion star, such as OB super giants or Be stars, can be excluded in AXPs. Moreover, no orbital motion signatures are present in their X-ray light curves.

Now we know 29 magnetars, 11 are reliably associated with supernova remnant shells, and an additional 2 have possible associations (Olausen \& Kaspi, 2014a,b). The large number of remnant associations is fully consistent with the great youth implied both by magnetar spin-down ages and by their proximity to the Galactic Plane. The associated remnants lack unusual properties when compared with shell remnants that harbor neutron stars with lower magnetic fields (Vink \& Kuiper 2006, Martin et al. 2014). This appears to be in conflict with the proposal of Duncan \& Thompson (1992) that magnetars form from neutron stars rotating with period $\sim 1 \mathrm{~ms}$ at birth, which assist a fast dynamo. The difficulty with this picture is that a neutron star with magnetic field $>10^{14} \mathrm{G}$ spinning at $1 \mathrm{~ms}$ quickly loses most of its rotational energy, releasing energy in excess of $10^{52} \mathrm{ergs}$, which is greater than the supernova explosion energy itself. It is therefore likely to be associated with either anomalously large shell remnants, or else no remnant at all, if it expanded sufficiently rapidly to dissipate on a timescale of a few hundred years. The normality of magnetar supernova remnants challenged the dynamo model and led to discussion of strong fossil fields from the progenitor star (Ferrario \& Wickramasinghe 2006, and e.g. Kaspi \& Beloborodov, 2017).

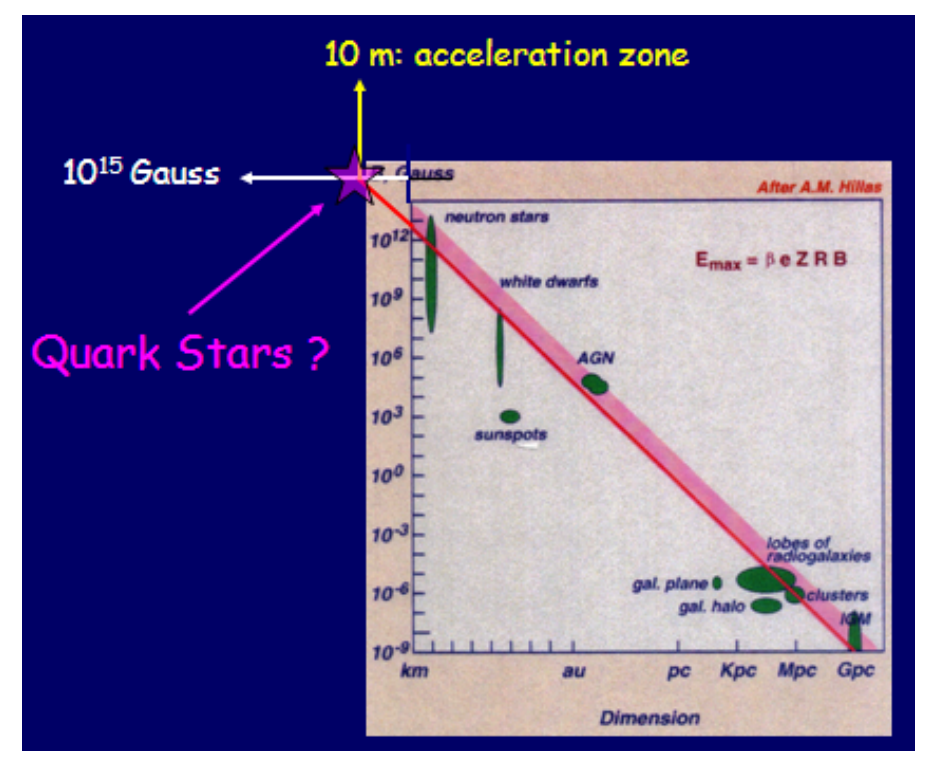

Figure 32: Magnetic field intensity vs dimensions of cosmic sources (adopted from Giovannelli \& SabauGraziati, 2006; courtesy of Todor Stanev, 2002, after Hillas, 1984. The extrapolation to $10^{15} \mathrm{G}$ provides a dimension of $\sim 10 \mathrm{~m}$ : the acceleration zone in a supercompact star (quark star?)

However, the nature of magnetar is not yet definitively proved. Giovannelli \& Sabau-Graziati (2006) speculated as follows: if magnetic fields of $\sim 10^{15} \mathrm{G}$ can be expected in order to explain the behaviour of magnetars, an almost 'obvious' consequence can be derived from the diagram 
Table 2: Pulse timing properties of magnetars and magnetar candidates (after Olausen \& Kaspi, 2014a and Kaspi \& Beloborodov, 2017).

\begin{tabular}{lllll}
\hline AXP \& SGR (Magnetars) & $\begin{array}{l}P_{\text {pulse }} \\
(\text { name) }\end{array}$ & $\begin{array}{l}\dot{P}_{\text {pulse }} \\
10^{-11} \mathrm{~s} \mathrm{~s}^{-1}\end{array}$ & $\begin{array}{l}\mathrm{B} \\
\left(10^{14} \mathrm{G}\right)\end{array}$ & $\begin{array}{l}\text { Spin-down } \\
\text { Age }(\mathrm{kyr})\end{array}$ \\
\hline CXOU J0110-721 (SMC) & 8.02 & 1.88 & 3.9 & 6.8 \\
4U 0142+614 & 8.69 & 0.20 & 1.3 & 68 \\
SGR 0418+5729 & 9.08 & 0.0004 & 0.061 & 36000 \\
SGR 0501+4516 & 5.76 & 0.582 & 1.9 & 16 \\
SGR 0526-66 (N49) & 8.05 & 3.8 & 5.6 & 3.4 \\
1E 1048.1-5937 & 6.46 & $\sim 2.25$ & 3.9 & 4.5 \\
PSR J1119-6127 & 0.41 & - & 4.1 & 1.6 \\
1E 1547.0-5408 & 2.07 & $\sim 4.77$ & 3.2 & 0.69 \\
PSR J1622-4950 & 4.33 & 1.7 & 2.7 & 4.0 \\
SGR 1627-41 (G337.0-0.1) & 2.59 & 1.9 & 2.2 & 2.2 \\
CXOU J164710.2-455216 & 10.61 & $<0.4$ & $<0.66$ & $>420$ \\
1RXS J170849.0-400910 & 11.00 & 1.91 & 4.6 & 9.1 \\
CXOU J171405.7-381031 & 3.82 & 6.40 & 5.0 & 0.95 \\
SGR J1745-2900 & 3.76 & 0.66 & 1.6 & 9.0 \\
SGR 1806-20 (G10.0-0.3) & 7.54 & $\sim 49.5$ & 20 & 0.24 \\
XTE J1810-197 & 5.54 & 0.78 & 2.1 & 11 \\
Swift J1822.3-1606 & 8.44 & 0.031 & 0.51 & 440 \\
SGR 1833-0832 & 7.56 & 0.35 & 1.6 & 34 \\
Swift J1834.9-0846 & 2.48 & 0.80 & 1,4 & 4.9 \\
1E1841-045 (Kes 73) & 11.78 & 3.93 & 6.9 & 4.7 \\
PSR J1846-0258 & 0.33 & - & 0.49 & 0.73 \\
3XMM J185246.6+003317 & 11.56 & - & $<0.41$ & $>1300$ \\
SGR 1900+14(G42.8+0.6) & 5.2 & 9.2 & 7.0 & 0.90 \\
SGR 1935+2154 & 3.24 & - & 2.2 & 3.6 \\
1E2259+586 (CTB 109) & 6.98 & 0.048 & 0.59 & 230 \\
\hline Magnetar Candidates & & & & \\
\hline SGR 0755-2933 & - & - & - & - \\
SGR 1801-23 & - & - & - & - \\
SGR 1808-20 & - & - & - & - \\
AX J 1818.8-1559 & - & - & - & - \\
AX J1845-026(G29.6+586) & 6.97 & - & - & - \\
SGR 2013+34 & - & - & - & - \\
\hline & & & & \\
\hline
\end{tabular}

magnetic field intensity versus the dimension of the relative cosmic source, like shown in Fig. 32. They extrapolated the value of $\mathrm{B}$ up to $10^{15} \mathrm{G}$; the correspondent dimension of the source is of $\sim 10 \mathrm{~m}$. This could be the dimension of the acceleration zone in a supercompact star, probably a quark star. If you construct a trap, the rat falls into it!

Table 2 shows the pulse timing properties of magnetars, the derived magnetic field intensity, the age (after Olausen \& Kaspi, 2014a and Kaspi \& Beloborodov, 2017), and their association with SNRs (after Giovannelli \& Sabau-Graziati, 2006).

The open questions about magnetars are numerous, namely: i) What are the distances of the Galactic magnetars? Then what is the Energetics? ii) What is the number-intensity relation for giant magnetar flares? iii) What are the SGR and AXP birth rate? What are their lifetimes? How 
many SGRs and AXPs are in the Milky Way? iv) What kind of supernova produces a SGR or an AXP? v) What is the relation between SGRs and AXPs? Does one evolve into the other, or are they separate manifestations of magnetars? vi) Are really the collapsed objects in SGRs and AXPs neutron stars? Alternatively, could they be quark stars? vii) How many other manifestations of magnetars exist?

In order to answer to these open questions, more sensitive instruments, more detailed theories, and more data (probably in the next 30 years) are necessary.

In the extensive and excellent reviews by Kitamoto et al. (2014) and by Kaspi \& Beloborodov (2017) most of the critical points about magnetars have been deeply discussed.

A large diversity of neutron stars has been discovered by multifrequency observations from the radio band to the X-ray and gamma-ray energy ranges. Among different manifestation of neutron stars - which include SGRs, AXPs, high-B pulsars (HBPs), high-E binaries (HEBs), rotating radio transients (RRATs), central compact objects (CCOs), rotation-powered radio pulsars (RPPs), and X-ray isolated neutron stars (XINSs) (Harding, 2013) - magnetars are the strongest magnetized objects.

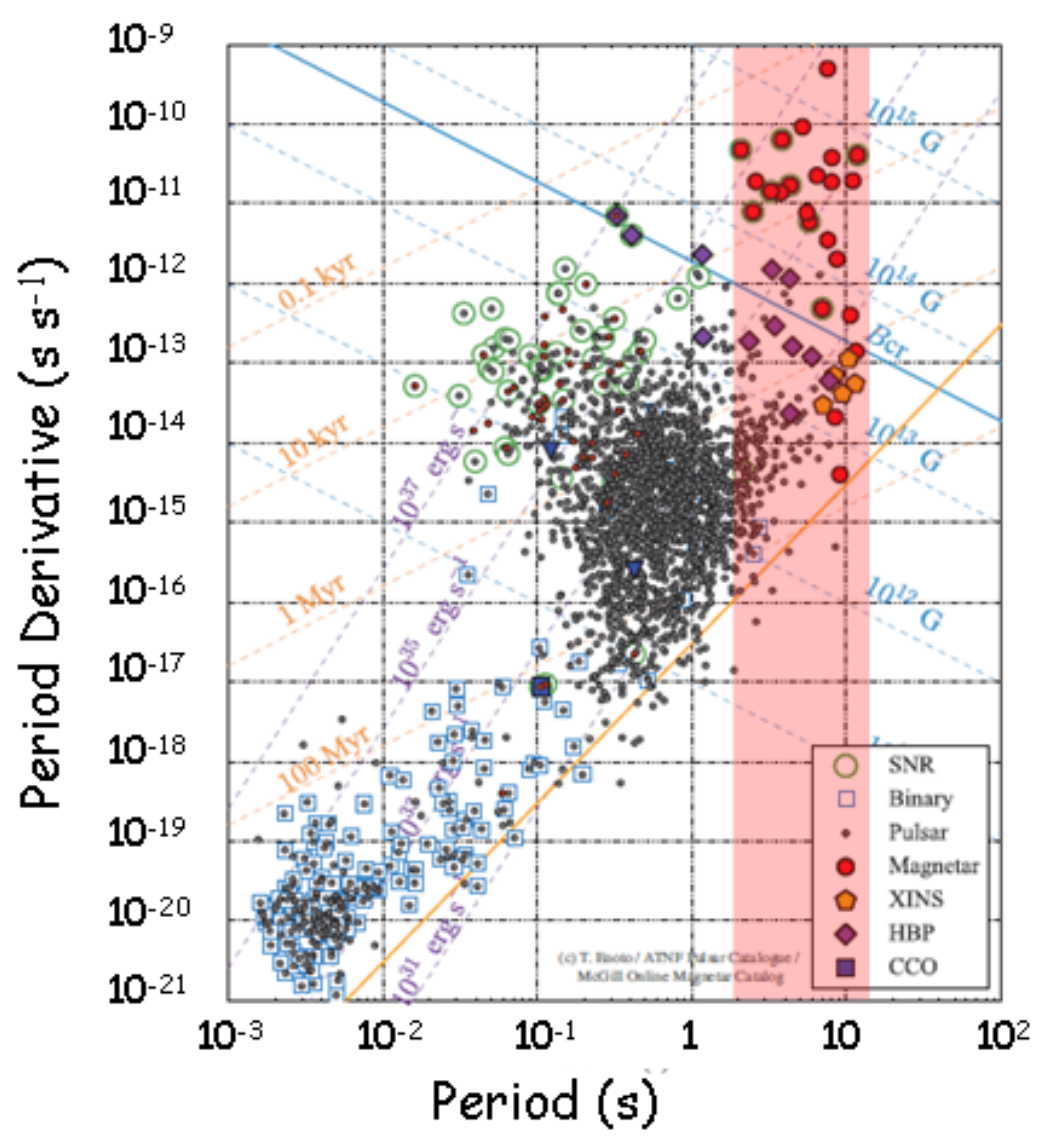

Figure 33: Magnetars and neutron stars on the $\mathrm{P}-\dot{\mathrm{P}}$ diagram. The light-red rectangle limits the range of magnetars periods (adapted from Enoto, 2018).

These various manifestations of neutron stars show different characteristics of rotation period $\mathrm{P}$ and its derivative $\dot{\mathrm{P}}$. The measurements of $\mathrm{P}$ and $\dot{\mathrm{P}}$ provide to estimate the dipole magnetic field 
strength $\mathrm{B}_{\mathrm{d}} \propto \sqrt{\mathrm{P} \dot{\mathrm{P}}}$ and characteristic age $\tau_{\mathrm{c}}=\mathrm{P} / 2 \dot{\mathrm{P}}$.

Figure 33 shows the $\mathrm{P}-\dot{\mathrm{P}}$ diagram (Enoto, 2018), where SGRs and AXPs are collectively called "magnetars" since their slow rotation $(\mathrm{P} \sim 2-12 \mathrm{~s})$ - with the exception of PSR J1119-6127 (P $=0.41 \mathrm{~s})$ and PSR J1846-0258 $(\mathrm{P}=0.33 \mathrm{~s})-$ and high period derivatives $\left(\dot{\mathrm{P}} \sim 10^{-13}-10^{-9} \mathrm{~s} \mathrm{~s}^{-1}\right)$ indicate high magnetic fields $\mathrm{B}=10^{14-15} \mathrm{G}$ and young characteristic age $\tau_{\mathrm{c}} \lesssim 10-100 \mathrm{kyr}$. To date, there are 29 known magnetars in the Milky Way and local universe (see Table 2).

It seems almost natural to think about a continuity among different classes of neutron star systems. However radio pulsations that have been observed from about 2000 neutron stars with weaker magnetic fields have never been detected from any of the known magnetars until the paper by Camilo et al. (2006) which showed that XTE J1810-197 — the first transient magnetar discovered (Ibrahim et al., 2004) - emits bright, narrow, highly linearly polarized radio pulses, observed at every rotation, thereby establishing that magnetars can be radio pulsars. Thus, these observations which link magnetars to ordinary radio pulsars, rule out alternative accretion models for AXPs, and provide a new window into the coronae of magnetars.

In the excellent review paper by Kaspi \& Beloborodov (2017) most of the critical points about magnetars have been deeply discussed. They concluded that: "The magnetar model has now been used to predict, naturally and uniquely, a wide variety of remarkable phenomena and behaviors in sources that once seemed highly anomalous. The now seamless chain of phenomenology from otherwise conventional radio pulsars through sources previously known for radically different behavior makes clear that these objects are one continuous family, with activity correlated with spin-inferred magnetic field strength. Recent advances in the physics of these objects, from the core through the crust and to the outer magnetosphere, hold significant promise.

The review by Bernardini (2015) discussed how the newly-born millisecond magnetars can compete with black holes as source of the GRB power, mainly with their rotational energy reservoir. They may be formed both in the core-collapse of massive stars, and in the merger of neutron star or white dwarf binaries, or in the accretion-induced collapse of a white dwarf, being thus a plausible progenitor for long and short GRBs, respectively. She reviewed the major observational evidences for the possible presence of a newly-born magnetar as the central engine for both long and short GRBs. She then discussed about the possibility that all GRBs are powered by magnetars, and she proposed a unification scheme that accommodates both magnetars and black holes, connected to the different properties and energetics of GRBs. Since the central engine remains hidden from direct electromagnetic observations, she reviewed the predictions for the GW emission from magnetars hosted from GRBs, and the observational perspectives with advanced interferometers.

\section{Future developments}

In this section we will try to clarify our point of view about future developments in astrophysics. Obviously we do not claim to be complete. We will simply discuss some examples that can clarify the enormous potential that will open up in the near future.

\subsection{Nuclear Reactions in Stars}

The knowledge of the cross-sections of nuclear reactions occurring in the stars appears as one of the most crucial points of all astroparticle physics. Direct measurements of the cross sections 
of the ${ }^{3} \mathrm{He}\left({ }^{4} \mathrm{He}, \gamma\right){ }^{7} \mathrm{Be}$ and ${ }^{7} \mathrm{Be}(\mathrm{p}, \gamma){ }^{8} \mathrm{Be}$ reactions of the $p p$ chain and ${ }^{14} \mathrm{~N}(\mathrm{p}, \gamma){ }^{15} \mathrm{O}$ reaction of the CNO-cycle will allow a substantial improvement in our knowledge on stellar evolution.

Wolschin (2003) published a very interesting paper about the history of the "Thermonuclear Processes in Stars and Stellar Neutrinos".

An impressive review about nuclear reactions (the pp chain and CNO cycles) has been published by Adelberger et al. (2011). They summarize and critically evaluate the available data on nuclear fusion cross sections important to energy generation in the Sun and other hydrogen-burning stars and to solar neutrino production. Recommended values and uncertainties are provided for key cross sections, and a recommended spectrum is given for ${ }^{8} \mathrm{~B}$ solar neutrinos. They also discuss opportunities for further increasing the precision of key rates, including new facilities, new experimental techniques, and improvements in theory. This review, which summarizes the conclusions of a workshop held at the Institute for Nuclear Theory, Seattle, in January 2009, is intended as a 10-year update and supplement to the reviews by Adelberger et al. (1998).

It is in the nature of astrophysics that many of the processes and objects one tries to understand are physically inaccessible. Thus, it is important that those aspects that can be studied in the laboratory be rather well understood.

One such aspect are the nuclear fusion reactions, which are at the heart of nuclear astrophysics: they influence sensitively the nucleosynthesis of the elements in the earliest stages of the universe and in all the objects formed thereafter, and control the associated energy generation, neutrino luminosity, and evolution of stars.

At the moment the LUNA (Laboratory for Underground Nuclear Astrophysics) is a new experimental approach for the study of nuclear fusion reactions based on an underground accelerator laboratory.

It is devoted to measure nuclear cross sections relevant in astroparticle physics. It is the most valuable experiment running underground in the Gran Sasso Laboratory of the INFN. Reviews about LUNA experiment have been published by Broggini et al. (2010; 2018).

The LUNA experiment deals with reproducing in the laboratory the nuclear reactions that generate most of the energy produced by the stars and that have allowed the synthesis of the elements within the stars and in the early Universe. These reactions are characterized by a very small probability (cross section) to the energies of astrophysical interest and are very difficult to measure in laboratories on the earth's surface where the cosmic background would mask the weak expected signal. In the last 25 years the LUNA collaboration has installed two accelerators in the LNGS (Laboratori Nazionali del Gran Sasso - INFN) underground laboratories and measured some key reactions in the hydrogen combustion cycle and primordial nucleosynthesis. In the near future a new accelerator will be installed that will also enable reactions of helium and carbon combustion cycles to be measured (Prati et al., 2017; Cavanna et al., 2018; Gustavino et al., 2019).

The LUNA collaboration has already measured with good accuracy the key reactions $\mathrm{D}(\mathrm{p}, \gamma)^{3} \mathrm{He}$, ${ }^{3} \mathrm{He}(\mathrm{D}, \mathrm{p}){ }^{4} \mathrm{He}$ and ${ }^{3} \mathrm{He}\left({ }^{4} \mathrm{He}, \gamma\right){ }^{7} \mathrm{Be}$. These measurements substantially reduce the theoretical uncertainty of $\mathrm{D},{ }^{3} \mathrm{He},{ }^{7} \mathrm{Li}$ abundances. The $\mathrm{D}\left({ }^{4} \mathrm{He}, \gamma\right){ }^{6} \mathrm{Li}$ cross section - which is the key reaction for the determination of the primordial abundance of ${ }^{6} \mathrm{Li}$ - has been measured (e.g. Gustavino, 2007, 2009, 2011, 2012, 2013), as well as that of ${ }^{2} \mathrm{H}(\alpha, \gamma){ }^{6} \mathrm{Li}$ (Anders et al., 2013), and ${ }^{2} \mathrm{H}(\alpha, \gamma){ }^{6} \mathrm{Li}$ (Anders et al., 2014). 
Other reactions fundamental for a better knowledge of stellar evolution have been studied by the LUNA experiment: e.g. ${ }^{17} \mathrm{O}(\mathrm{p}, \gamma){ }^{18} \mathrm{~F}$ (Scott et al. 2012); ${ }^{25} \mathrm{Mg}(\mathrm{p}, \gamma){ }^{26} \mathrm{Al}$ (Strieder et al., 2012) ${ }^{25} \mathrm{Mg}(\mathrm{p}, \gamma){ }^{26} \mathrm{Al}$ (Straniero et al., 2013); ${ }^{17} \mathrm{O}(\mathrm{p}, \gamma){ }^{18} \mathrm{~F}$ (Di Leva et al., 2014).

Cavanna et al. (2015) studied with the LUNA experiment the ${ }^{22} \mathrm{Ne}(\mathrm{p}, \gamma)^{23} \mathrm{Na}$ reaction that takes part in the neon-sodium cycle of hydrogen burning. This cycle affects the synthesis of the elements between ${ }^{20} \mathrm{Ne}$ and ${ }^{27} \mathrm{Al}$ in asymptotic giant branch stars and novae. They found a new reaction rate a factor of 5 higher than the recent evaluation at temperatures relevant to novae and asymptotic giant branch stars nucleosynthesis.

Depalo et al. (2016) performed direct measurements of the ${ }^{22} \mathrm{Ne}(\mathrm{p}, \gamma)^{23} \mathrm{Na}$ resonances with the LUNA experiment. Based on the present experimental data and also previous literature data, an updated thermonuclear reaction rate is provided in tabular and parametric form. The new-reaction rate is significantly higher than previous evaluations at temperatures of 0.08-0.3 GK.

The ${ }^{17} \mathrm{O}(\mathrm{p}, \alpha)^{14} \mathrm{Na}$ reaction plays a key role in various astrophysical scenarios, from asymptotic giant branch stars to classical novae. It affects the synthesis of rare isotopes such as ${ }^{17} \mathrm{O}$ and ${ }^{18} \mathrm{~F}$, which can provide constraints on astrophysical models. Bruno et al. (2016) performed direct determination of the resonance strength $\mathrm{E}_{\mathrm{R}}=64.5 \mathrm{keV}$ of that reaction at the LUNA accelerator. They found a factor of 2 increase in the reaction rate at astrophysical temperatures relevant to shell hydrogen burning in red giant and asymptotic giant branch stars. The new rate implies lower ${ }^{17} \mathrm{O} /{ }^{16} \mathrm{O}$ ratios, with important implications on the interpretation of astrophysical observable quantities from these stars, as deeply discussed by Straniero et al. (2017).

In order to further remark the importance of LUNA measurement, we would like to mention the crucial problem of the correct prediction of the abundances of the light nuclides produced during the epoch of Big Bang Nucleosynthesis (BBN) which is one of the main topics of modern cosmology. Trezzi et al. (2017) report results about the cross section of the ${ }^{2} \mathrm{H}(\alpha, \gamma)^{6} \mathrm{Li}$ reaction that controls ${ }^{6} \mathrm{Li}$ production in the Big Bang. The cross section has been directly measured at the energies of interest for BBN for the first time, at center-of-mass energy $\mathrm{E}_{\mathrm{cm}}=80,93,120$, and 133 $\mathrm{keV}$. They found that the ${ }^{2} \mathrm{H}(\alpha, \gamma){ }^{6} \mathrm{Li}$ thermonuclear reaction rate is even lower than previously reported, thus increasing the discrepancy between predicted Big Bang ${ }^{6} \mathrm{Li}$ abundance and the amount of primordial ${ }^{6} \mathrm{Li}$ inferred from observations.

A general data base for Experimental Nuclear Reaction Data (EXFOR) can be found in: https://www-nds.iaea.org/exfor/exfor.htm.

\subsection{Gaia mission}

While ESA's Gaia mission has been surveying more than one billion stars from space, scientific astronomical community have been regularly monitoring the satellite's position in the sky with telescopes across the world, including the European Southern Observatory in Chile, to further refine Gaia's orbit and ultimately improve the accuracy of its stellar census (e.g. Prusti et al., 2016).

Figure 34 shows a schematic comparison between the Gaia observatory potentiality with respect to those of the Hipparcos observatory.

With its all-sky survey of the position, brightness and motion of over one billion stars in our Milky Way galaxy, Gaia provided a large dataset to search for exoplanets - planets orbiting stars other than the Sun. These will be uncovered by monitoring tiny changes in a star's position and 


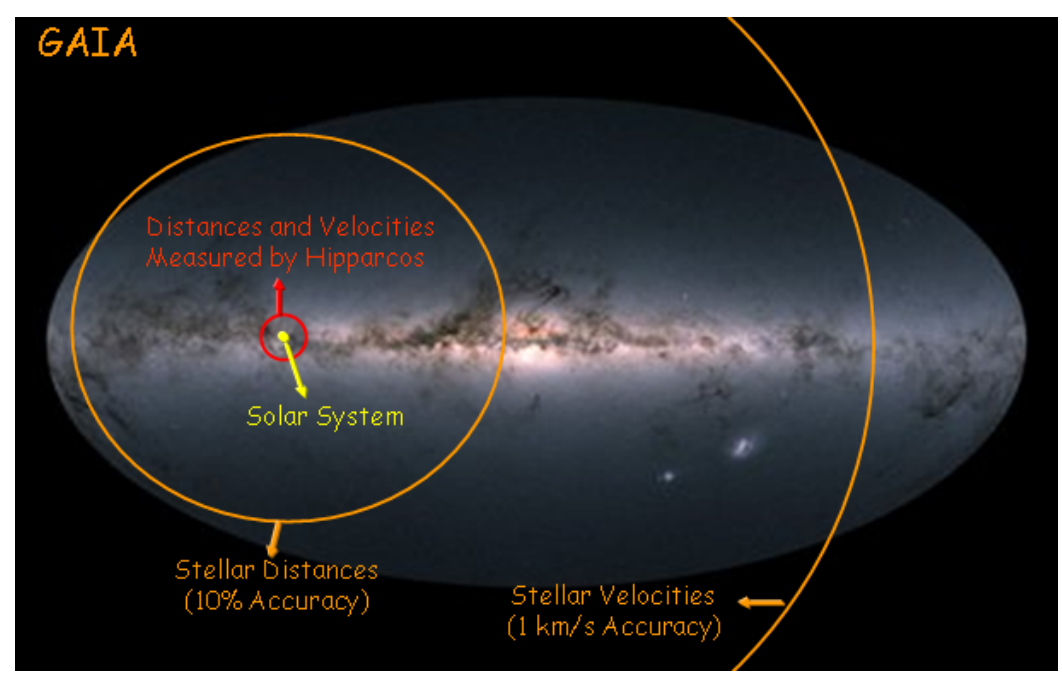

Figure 34: Comparison between Gaia and Hipparcos capabilities.

motion caused by the gravitational pull of one or more planets around it, and by looking for dips in the stellar light caused by a planet transiting in front of its parent star (https://sci.esa.int/web/gaia/158784-exoplanets).

The mass of the Milky Way has been one of the most fundamental measurements to be performed in modern astrophysics. However, despite decades of intense effort, even the best available estimates of the Milky Way's mass disagree wildly. Hubble \& Gaia accurately weigh the Milky Way. In a striking example of multi-mission astronomy, measurements from the NASA/ESA Hubble Space Telescope and the ESA Gaia mission have been combined to improve the estimate of the mass of our home Galaxy: $\sim 1.12 \times 10^{12} \mathrm{M}_{\odot}$ within a radius of $\sim 12 \mathrm{kpc}$ from the galactic center. The mass attributed to the stars is $\sim 4.99 \times 10^{10} \mathrm{M}_{\odot}$ (Cautun et al., 2019).

Wang et al. (2019) performed an extensive review of the numerous studies and methods used to determine the total mass of the Milky way.

Previous estimates of the mass of the Milky Way ranged from $5 \times 10^{11}$ to $3 \times 10^{12} \mathrm{M}_{\odot}$. This huge uncertainty arose primarily from the different methods used for measuring the distribution of dark matter - which makes up about $90 \%$ of the mass of the galaxy.

Important synergy between distances measured by Gaia and knowledge of cross sections of fundamental reactions occurring in the interior of stars (like results coming from the LUNA experiment) can provide a new vision of the real luminosity of stars, with strong implications on the stellar evolution theory.

\subsection{The Cherenkov Telescope Array}

The Cherenkov Telescope Array (CTA) will be a next-generation ground-based observatory for very high energy gamma-ray astronomy. It will consist of two arrays of dishes, a southernhemisphere array at ESO's Paranal Observatory (e.g. Hofmann et al., 2017) and a northern array on the island of La Palma, Spain (e.g. Williams et al., 2019).

Figure 35 shows the CTA Telescopes in Southern Hemisphere (http : //www.eso.org; Credit: CTA/M-A. Besel/IAC (G.P. Diaz)/ESO). This image illustrates all three classes of the telescopes 


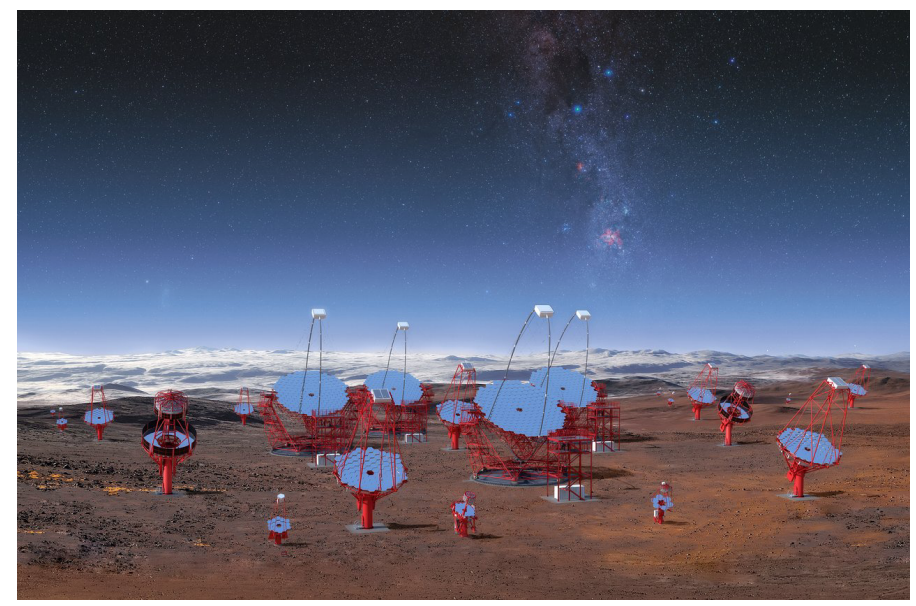

Figure 35: The CTA Telescopes in Southern Hemisphere (http : //www.eso.org; Credit: CTA/M-A. Besel/IAC (G.P. Diaz)/ESO).

planned for the southern hemisphere at ESO's Paranal Observatory, as viewed from the center of the array. This rendering is not an accurate representation of the final array layout, but it illustrates the enormous scale of the CTA telescopes and the array itself.

Figure 36 shows:(upper panel) a sketch of the science potential of the current experiments in comparison with the forthcoming CTA (Paredes \& Persic, 2009); (lower panel) the sensitivity of most of the high energy experiments, including that of CTA. It is impressive to note that the sensitivity of the CTA at $1 \mathrm{TeV}$ is $1 \mathrm{mCrab}$ (after http://www.cfar.ie/research-areas.html).

A complete description of the Science that can be performed with the CTA has been published by Acharya et al. (2019).

\subsection{ESO-Extremely Large Telescope}

Astronomy is experiencing a golden era. The past decade alone has brought amazing discoveries that have excited people from all walks of life, from finding planets around Proxima Centauri, the nearest star to the Sun, to the first image of a black hole.

Extremely large telescopes are considered worldwide to be one of the highest priorities in ground-based astronomy. They will vastly advance astrophysical knowledge, allowing detailed studies of subjects including planets around other stars, the first objects in the Universe, supermassive black holes, and the nature and distribution of the dark matter and dark energy which dominate the Universe (https://www.eso.org/public/italy/teles-instr/elt/).

Since 2005 ESO has been working with its community and industry to develop an extremely large optical/infrared telescope, named the ELT (Extremely Large Telescope). This revolutionary new ground-based telescope concept will have a 39-meter main mirror and will be the largest optical/near-infrared telescope in the world: "the world's biggest eye on the sky" (Gilmozzi \& Spyromilio, 2007, 2009; Gilmozzi, 2008, 2009; Gilmozzi \& Kissler-Patig, 2011; McPhersonet et al., 2012; Liske, Padovani \& Patig, 2012; Ramsay et al., 2016; Padovani, 2018).

The final approval of E-ELT occurred at ESO on December 3, 2014 (de Zeeuw, Tamai \& Liske, 2014). On May 25, 2016 ESO Signs Largest Ever Ground-based Astronomy Contract for 

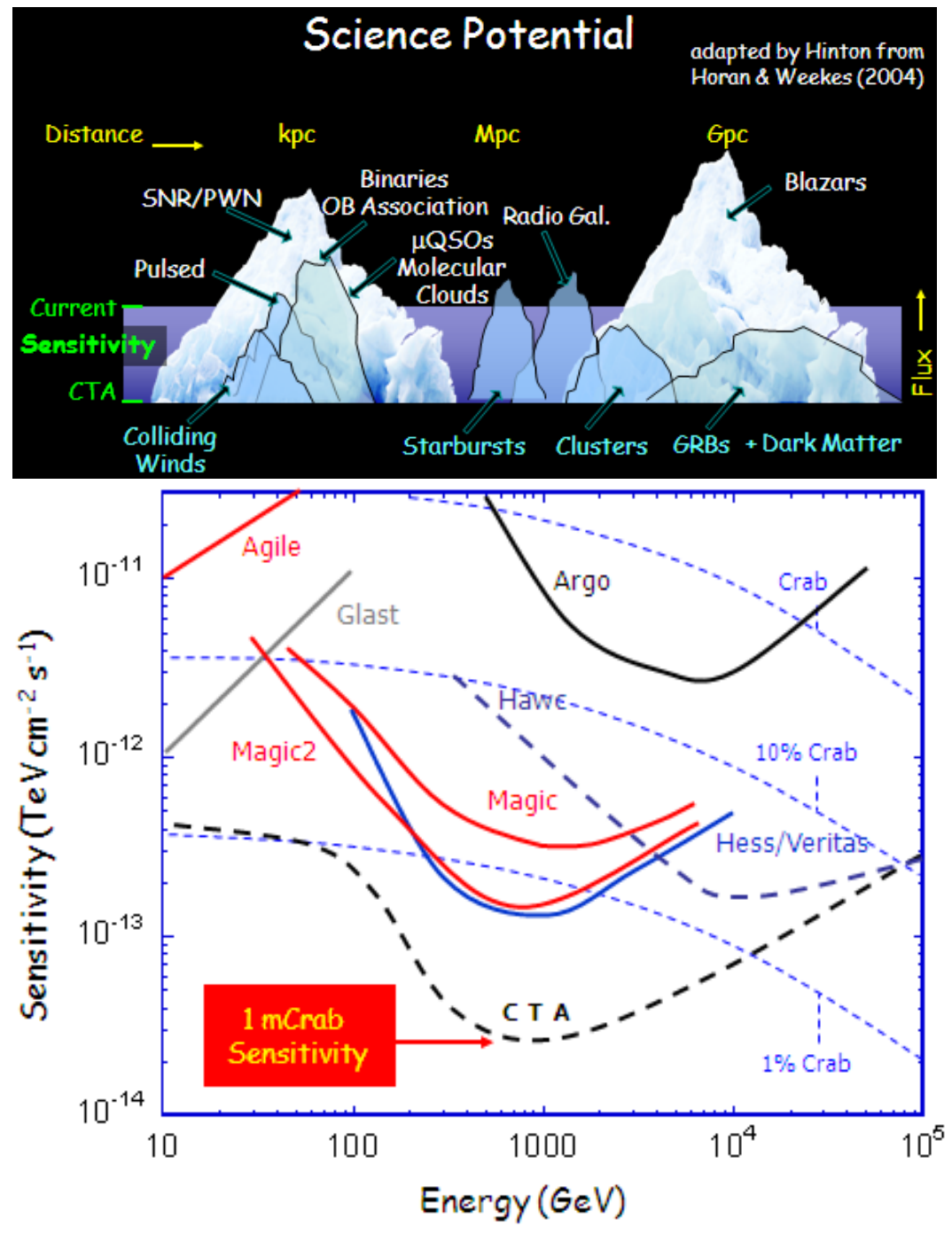

Figure 36: Upper panel: New horizons in VHE astrophysics with the advent of the CTA (Paredes \& Persic, 2009); Lower panel: The sensitivity of most of the HE experiments versus energy (after http://www.cfar.ie/research-areas.html).

ELT Dome and Telescope Structure (eso1617 - Organisation Release).

An expanded view of the Universe (Science with the European Extremely Large Telescope) can be found in http://www.eso.org/sci/facilities/eelt/docs/.

Figure 37 shows the dome of the E-ELT in La Silla observatory (left panel) and the grandeur of the telescope in comparison with the pyramids (right panel) (https://www.eso.org/public/telesinstr/elt/; Gilmozzi, 2013, respectively).

It is important to mention a white paper about ESO-Athena synergy (Padovani et al., 2017).

\subsection{Canadian Hydrogen Intensity Mapping Experiment: CHIME}

The Canadian Hydrogen Intensity Mapping Experiment (CHIME) is an interferometric radio telescope located at the Dominion Radio Astrophysical Observatory (DRAO) in British Columbia, 

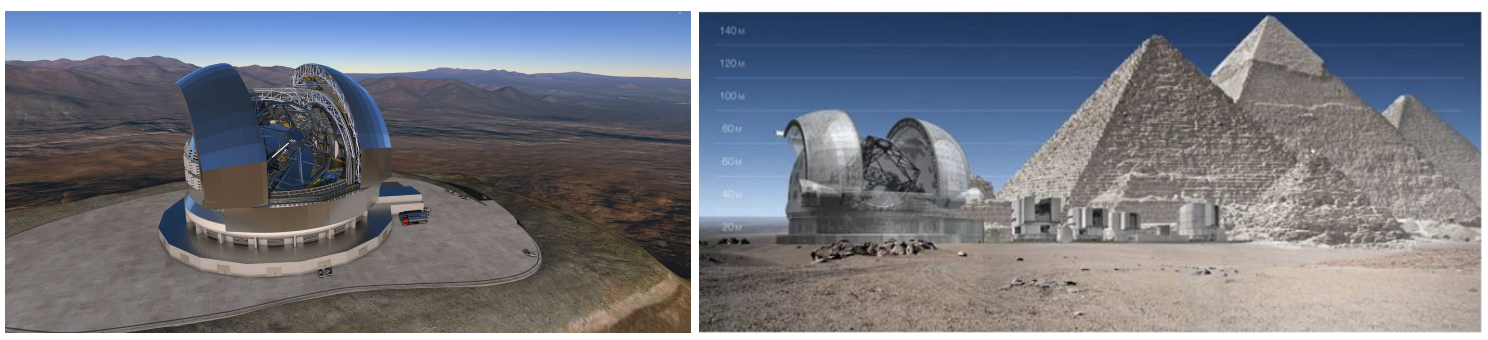

Figure 37: Left panel: the dome of the E-ELT in La Silla observatory (https://www.eso.org/public/telesinstr/elt/); Right panel: the grandeur of the telescope in comparison with the pyramids (Gilmozzi, 2013).

Canada. It consists of four 100 x 20-meter semi-cylinders on which 1024 dual-polarization radio receivers sensitive to frequencies between $400-800 \mathrm{MHz}$ are installed. The main aim of the project is to deepen the cosmological knowledge of the structure of the young universe, dark energy and the standard $\lambda \mathrm{CDM}$ model, which better reproduces the observations of cosmology following the Big Bang (e.g. Bandura et al., 2014). The telescope was inaugurated on 7 September 2017 (Murray, 2018: http://www.astronomy.com/news/2018/03/chime-begins-its-cosmic-search).

CHIME is a digital telescope, which means all of its "imaging" is done digitally by software. CHIME has a 4 x 256 array of antennas and can form all 1024 independent beams in real time. Raw sensitivity is the same as the same 1024 single-feed radio telescopes (Smith, 2019).

A usual dish of a radio telescope is replaced by an array of antennas whose signals are digitized. by summing signals with appropriate delays, can simulate the dish in software, and focus on part of the sky. The telescope can be repointed by changing delays.

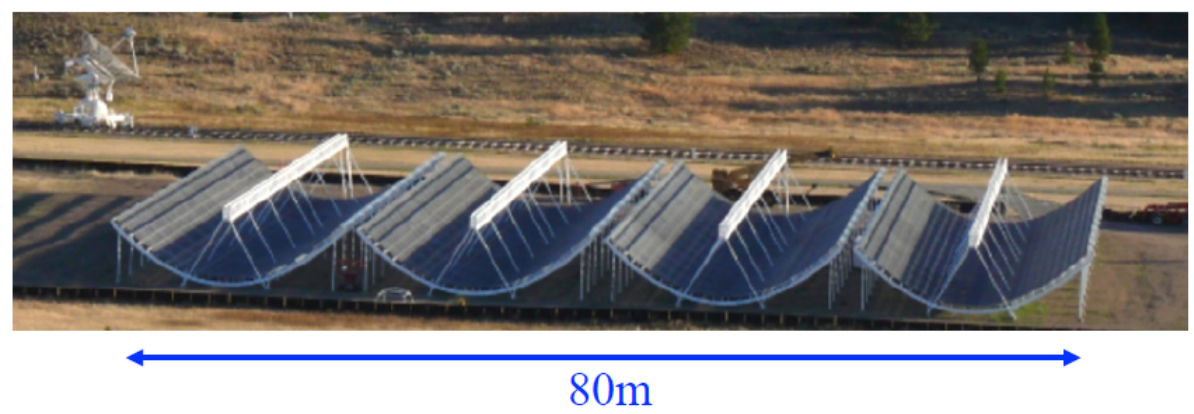

Figure 38: Canadian Hydrogen Intensity Mapping Experiment (Smith, 2019).

Starting from the preliminary detections of $\mathrm{CO},[\mathrm{CII}], \mathrm{Ly} \alpha$ and low-redshift $21 \mathrm{~cm}$, and a host of experiments set to go online in the next few years, the field is rapidly progressing on all fronts, with great anticipation for a flood of new exciting results, Kovetz et al. (2017) after reviewing the first detections reported to date, they survey the experimental landscape, presenting the parameters and capabilities of relevant instruments such as COMAP, mmIMe, AIM-CO, CCATp, TIME, CONCERTO, CHIME, HIRAX, HERA, STARFIRE, MeerKAT/SKA and SPHEREx. Finally, they describe recent theoretical advances: different approaches to modeling line luminosity functions, several techniques to separate the desired signal from foregrounds, statistical methods to analyze the data, and frameworks to generate realistic intensity map simulations. 


\section{6 e-ASTROGAM Observatory}

e-ASTROGAM (enhanced ASTROGAM) is a breakthrough Observatory space mission, with a detector composed by a Silicon tracker, a calorimeter, and an anticoincidence system, dedicated to the study of the non-thermal Universe in the photon energy range from $0.3 \mathrm{MeV}$ to $3 \mathrm{GeV}$ the lower energy limit can be pushed to energies as low as $150 \mathrm{keV}$, albeit with rapidly degrading angular resolution, for the tracker, and to $30 \mathrm{keV}$ for calorimetric detection. The mission is based on an advanced space-proven detector technology, with unprecedented sensitivity, angular and energy resolution, combined with polarimetric capability. Thanks to its performance in the $\mathrm{MeV}-\mathrm{GeV}$ domain, substantially improving its predecessors, e-ASTROGAM will open a new window on the non-thermal Universe, making pioneering observations of the most powerful Galactic and extragalactic sources, elucidating the nature of their relativistic outflows and their effects on the surroundings (De Angelis, et al., 2017).

Figure 39 shows: in the upper panel the sensitivity of e-ASTROGAM compared with those of the past, present and future experiments; in the lower panel the compilation of measurements of extragalactic sources between $1 \mathrm{keV}$ and $820 \mathrm{GeV}$, The semitransparent band indicates the energy region in which e-ASTROGAM will strongly improve on present knowledge (De Angelis, et al., 2017).

\subsection{The THESEUS mission}

The THESEUS mission is designed to vastly increase the discovery space of the high energy transient phenomena over the entirety of cosmic history (http://www.isdc.unige.ch/theseus/).

Amati et al. (2018) describe the main scientific goals of the proposed mission that are to:

i) Explore the Early Universe (cosmic dawn and reionization era) by unveiling a complete census of the Gamma-Ray Burst (GRB) population in the first billion years.

ii) Perform an unprecedented deep monitoring of the $X$-ray transient Universe.

Figure 40 shows the GRASP FoV versus Effective Area) as function of energy (upper panel) and the cumulative distribution of GRBs with redshift determination as a function of the redshift for Swift (in $10 \mathrm{yr}$ ) and the prediction for THESEUS (in $3 \mathrm{yr}$ ) (lower panel). A deep description of Theseus mission can be found in THESEUS: CDF Study - Executive Summary (https://sci.esa.int/documents/34923/36148/1567260367145-THESEUS_CDF_IFP_2018_Summary.pdf).

\subsection{The James Webb Space Telescope}

The James Webb Space Telescope (JWST) will be a giant leap forward in our quest to understand the Universe and our origins. JWST will examine every phase of cosmic history: from the first luminous glows after the Big Bang to the formation of galaxies, stars, and planets to the evolution of our own solar system (https://jwst.nasa.gov/science.html). Webb often gets called the replacement for Hubble, but we prefer to call it a successor. After all, Webb is the scientific successor to Hubble; its science goals were motivated by results from Hubble. Hubble's science pushed us to look to longer wavelengths to "go beyond" what Hubble has already done. In particular, more distant objects are more highly redshifted, and their light is pushed from the UV and optical into the near-infrared. Thus observations of these distant objects (like the first galaxies formed in the Universe, for example) requires an infrared telescope. 

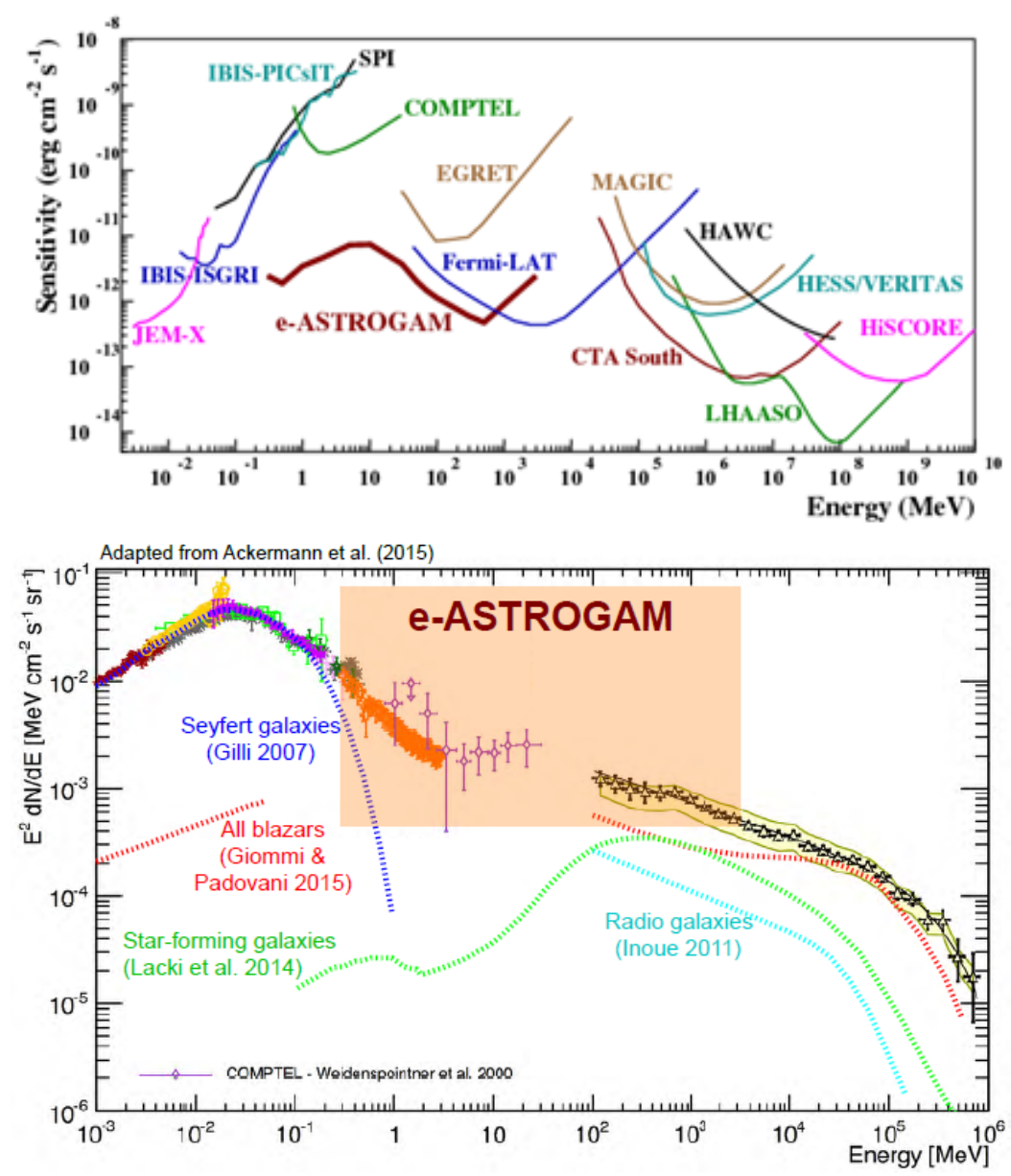

Figure 39: Upper panel: the sensitivity of e-ASTROGAM compared with those of the past, present and future experiments; lower panel: the compilation of measurements of extragalactic sources between $1 \mathrm{keV}$ and $820 \mathrm{GeV}$, The semitransparent band indicates the energy region in which e-ASTROGAM will strongly improve on present knowledge (De Angelis, et al., 2017).

This is the other reason that Webb is not a replacement for Hubble; its capabilities are not identical. Webb will primarily look at the Universe in the infrared, while Hubble studies it primarily at optical and ultraviolet wavelengths (though it has some infrared capability). Webb also has a much bigger mirror than Hubble. This larger light collecting area means that Webb can peer farther back into time than Hubble is capable of doing. Hubble is in a very close orbit around the earth, while Webb will be 1.5 million kilometers $(\mathrm{km})$ away at the second Lagrange (L2) point.

The backplane is the spine of the telescope and is the structure on which the mirrors and instruments will be mounted, as showed in Fig 41 (jwst.nasa.gov). The Potential Science with JWST can be found in a series of white papers at http://www.stsci.edu/jwst/about-jwst/history/white-papers.

A complete description of the JWST potential science is reported in the review paper by Gardner et al. (2006) A synthesis of the potential science that can be performed by the JWST is reported in the paper by Gardner (2009).

After several delays, the JWST is scheduled for launch in 2021. Indeed, on March 27, 2018, 

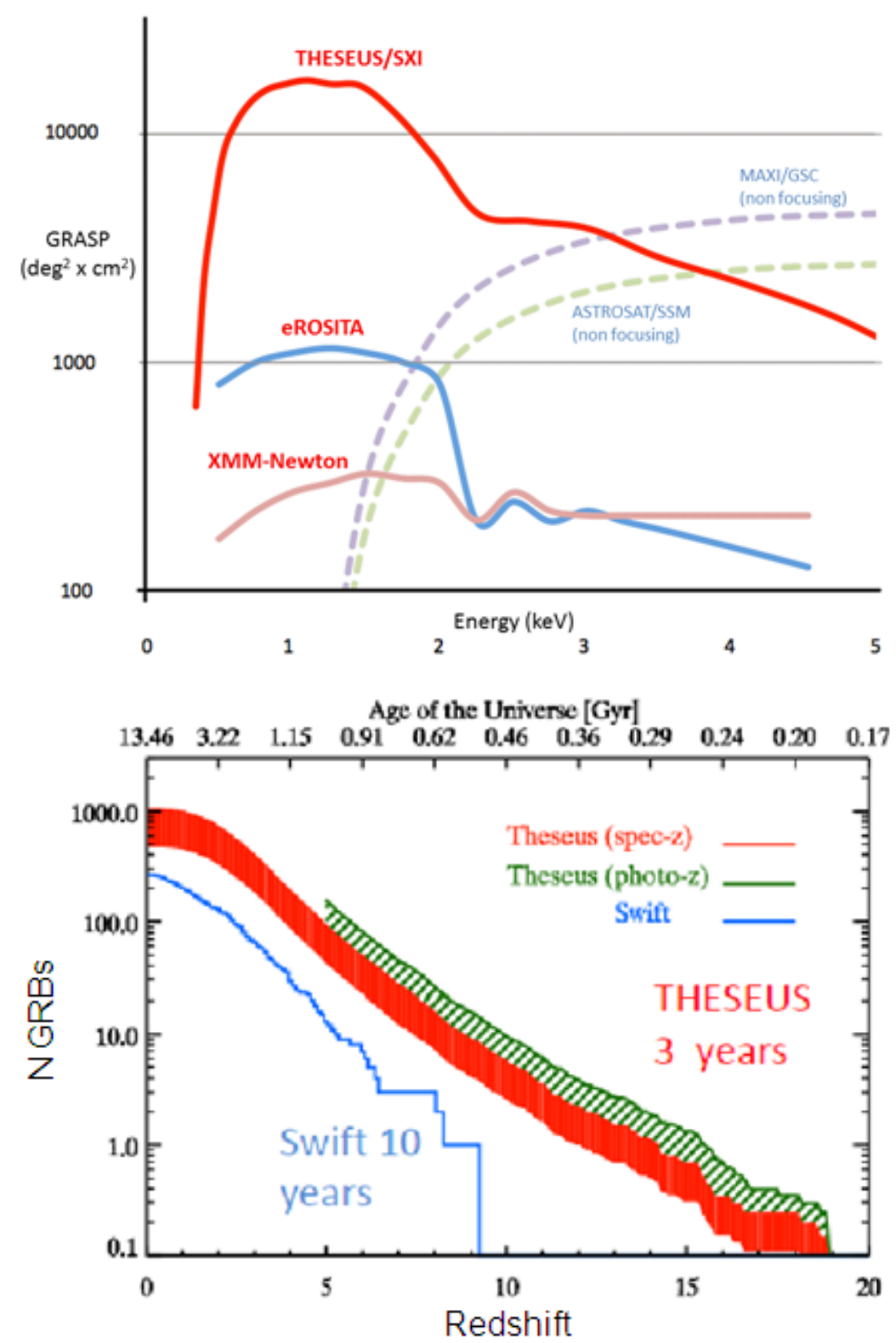

Figure 40: (Upper panel: the GRASP (FoV versus Effective Area) as function of energy; lower panel: the cumulative distribution of GRBs with redshift determination as a function of the redshift for Swift (in $10 \mathrm{yr}$ ) and the prediction for THESEUS (in $3 \mathrm{yr}$ ) (http://www.isdc.unige.ch/theseus/).

JWST launch delayed to at least May 2020 as issues with the spacecraft element require further testing, and on June 27, 2018, JWST launch postponed to March 30, 2021 - planned launch as of June 2018 - based on recommendations by an Independent Review Board.

\subsection{The GAMMA-400 gamma-ray telescope}

The GAMMA-400 gamma-ray telescope: the next absolutely necessary step in the development of extraterrestrial high-energy $\gamma$-ray astronomy is the improvement of the physical and 


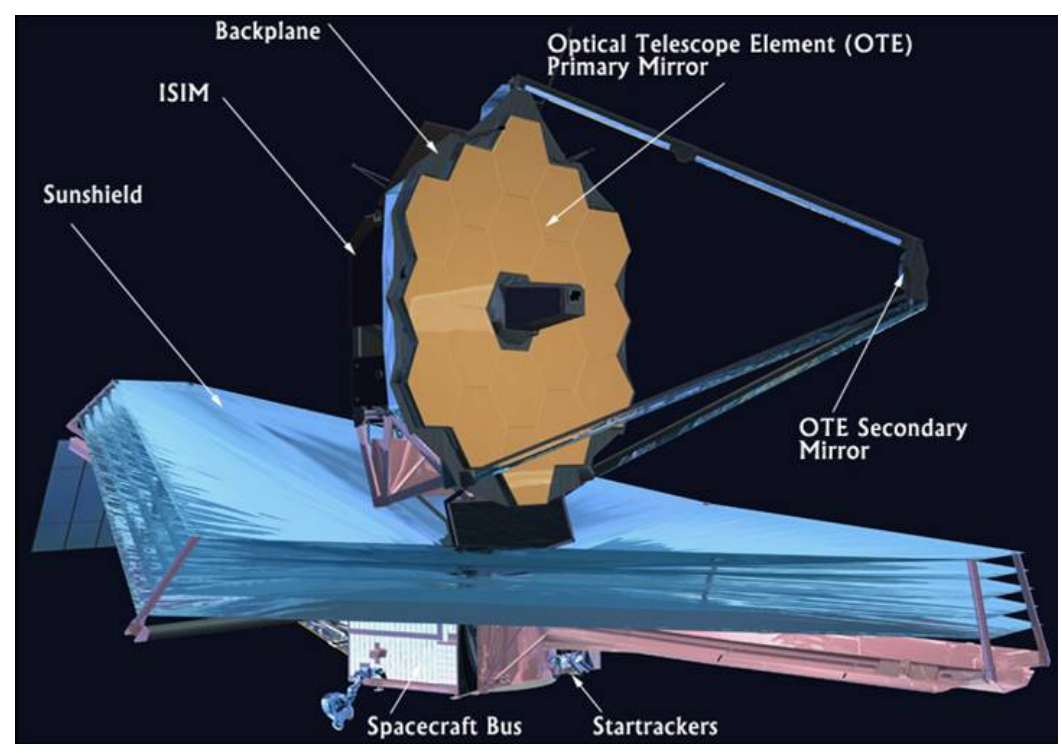

Figure 41: Sketch of the JWST (jwst.nasa.gov).

technical characteristics of $\gamma$-ray telescopes, especially the angular and energy resolutions. Such a new generation telescope will be GAMMA-400, which will be installed onboard the Russian space observatory (Cumani et al., 2015; Galper, Topchiev \& Yurkin, 2018).

The GAMMA-400 gamma-ray telescope is intended to measure the fluxes of gamma-rays and cosmic-ray electrons and positrons in the energy range from $100 \mathrm{MeV}$ to several TeV. Such measurements concern the following scientific tasks: investigation of point sources of gamma-rays, studies of the energy spectra of Galactic and extragalactic diffuse emission, studies of gamma-ray bursts and gamma-ray emission from the Sun, as well as high precision measurements of spectra of high-energy electrons and positrons. Also the GAMMA- 400 instrument provides the possibility for protons and nuclei measurements up to knee. But the main goal for the GAMMA-400 mission is to perform a sensitive search for signatures of dark matter particles in high-energy gamma-ray emission (Topchiev et al., 2017a, 2019 and the references therein).

The GAMMA-400 will operate in the highly elliptic orbit continuously for a long time with the unprecedented angular $\left(\sim 0.01^{\circ}\right.$ at $\left.\mathrm{E}_{\gamma}=100 \mathrm{GeV}\right)$ and energy $\left(\sim 1 \%\right.$ at $\left.\mathrm{E}_{\gamma}=100 \mathrm{GeV}\right)$ resolutions better than the Fermi-LAT, as well as ground $\gamma$-ray telescopes, by a factor of 5-10. GAMMA-400 will permit to resolve $\gamma$-rays from annihilation or decay of dark matter particles, identify many discrete sources (many of which are variable), to clarify the structure of extended sources, to specify the data on the diffuse emission (Topchiev et al., 2017b). Figure 42 shows the energy and angular resolutions of GAMMA-400 versus other experiments (Topchiev et al., 2015)).

\subsection{The Large Hadron Collider (LHC)}

The Large Hadron Collider (LHC) is the world's largest and most powerful particle accelerator (https://home.cern/topics/large-hadron-collider). It first started up on 10 September 2008, and remains the latest addition to CERN's accelerator complex. The LHC consists of a $27 \mathrm{~km}$ ring of superconducting magnets with a number of accelerating structures to boost the energy of the particles along the way. Figure 43 shows a partial view of the tunnel hosting the accelerator. 

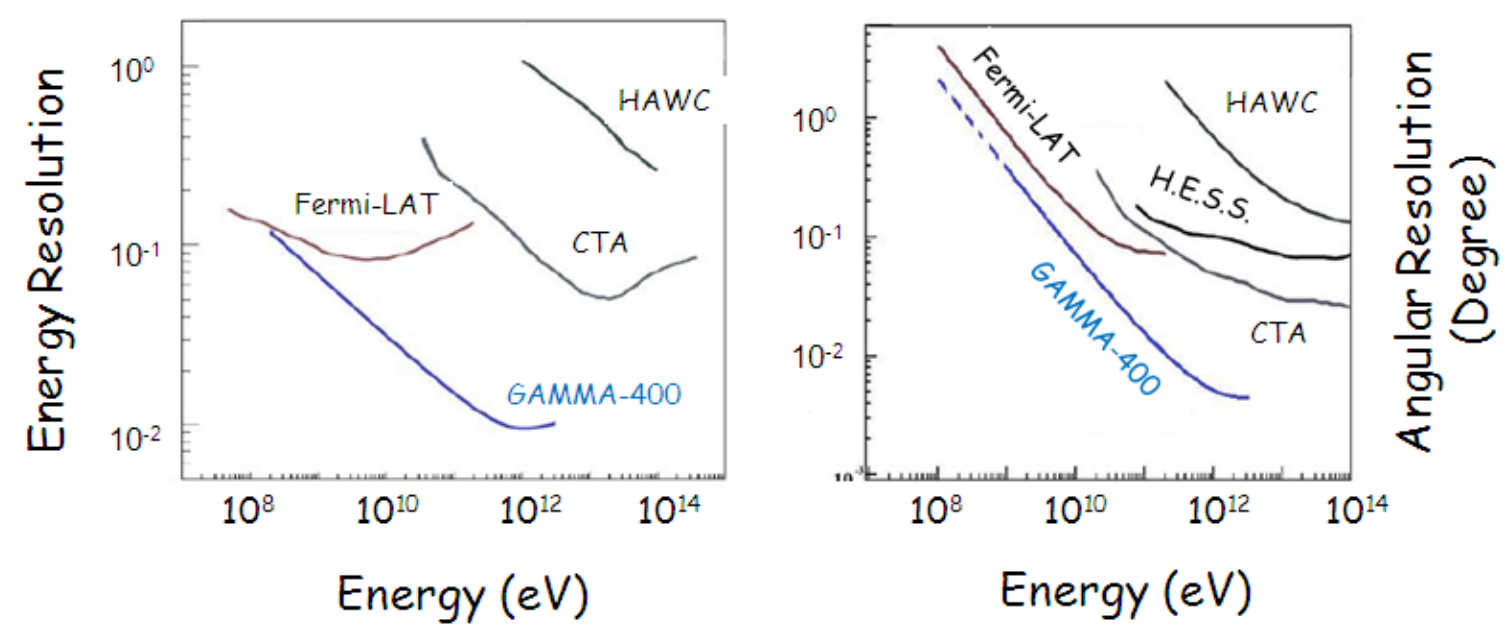

Figure 42: Energy and angular resolutions of GAMMA-400 versus other experiments (adapted from Topchiev et al., 2015).

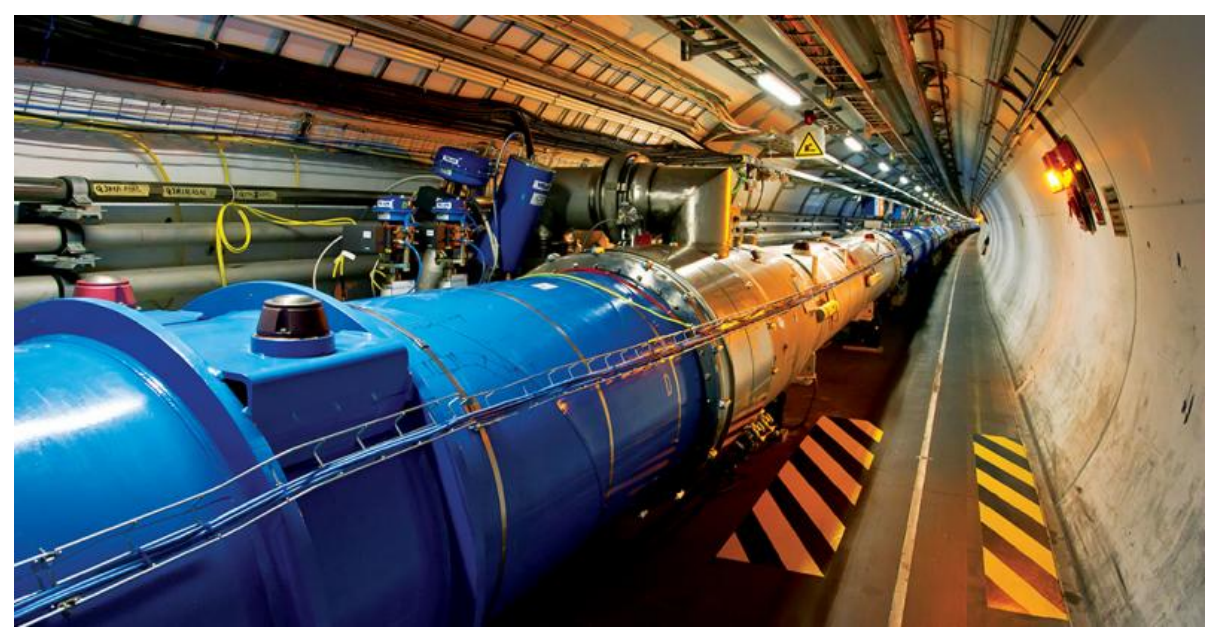

Figure 43: The Large Hadron Collider is the world's largest and most powerful particle accelerator (Image: CERN, at https://home.cern/topics/large-hadron-collider).

The LHC will answer some of the fundamental open questions in physics, concerning the basic laws governing the interactions and forces among the elementary objects, the deep structure of space and time, and in particular the interrelation between quantum mechanics and general relativity. Data is also needed from high-energy particle experiments to suggest which versions of current scientific models are more likely to be correct - in particular to choose between the Standard Model and Higgs-less model and to validate their predictions and allow further theoretical development. Many theorists expect new physics beyond the Standard Model to emerge at the TeV energy level, as the Standard Model appears to be unsatisfactory. Issues explored by LHC collisions include i) the mass of elementary particles being generated by the Higgs mechanism; ii) supersymmetry, an extension of the Standard Model and Poincaré symmetry; iii) extra dimensions, as predicted by various models based on string theory; iv) the nature of the dark matter that appears to account for $\sim 27 \%$ of the mass-energy of the universe; v) answer to the question if the electroweak force 
and the strong nuclear force are just different manifestations of one universal unified force, as predicted by various Grand Unification Theories; vi) why the fourth fundamental force (gravity) is so many orders of magnitude weaker than the other three fundamental forces; vii) are there additional sources of quark flavour mixing, beyond those already present within the Standard Model?; viii) why are there apparent violations of the symmetry between matter and antimatter?; ix) what are the nature and properties of quark-gluon plasma, thought to have existed in the early universe and in certain compact and strange astronomical objects today?

\subsection{The BICEP (Background Imaging of Cosmic Extragalactic Polarization) and the Keck Array}

The BICEP (Background Imaging of Cosmic Extragalactic Polarization) and the Keck Array are a series of cosmic microwave background (CMB) experiments. They aim to measure the polarization of the $\mathrm{CMB}$; in particular, measuring the B-mode of the CMB. The experiments have had four generations of instrumentation, consisting of BICEP1, BICEP2, the Keck Array, and BICEP3. These experiments are observing from the South Pole, and their aims are to discover signatures of Inflation by actually detecting the Cosmic Gravitational Background (CGB) via its weak imprint as the unique $\mathrm{B}$-mode polarization signature of the $\mathrm{CMB}$, directly probing the Universe at an earlier time than ever before. Each generation represents a large increase in sensitivity to B-mode polarization. BICEP1 observed from 2006-2008 with 98 detectors, BICEP2 began observing in the beginning of 2010 with 512 detectors, and the first three of five Keck Array telescopes began observing in the beginning of 2011, each with 512 detectors. The final two Keck Array receivers were deployed during the summer season of 2012. BICEP3, with a total of 2,560 detectors, has been operational since May 2016.

Figure 44 shows the installation of the BICEP experiment at the South Pole which is near the middle of the Antarctic plateau, the driest environment on the planet.

The main goal of BICEP experiments is to test the validity of the theory of the Inflation (Keating et al., 2003a,b; Ogburn IV et al., 2010).

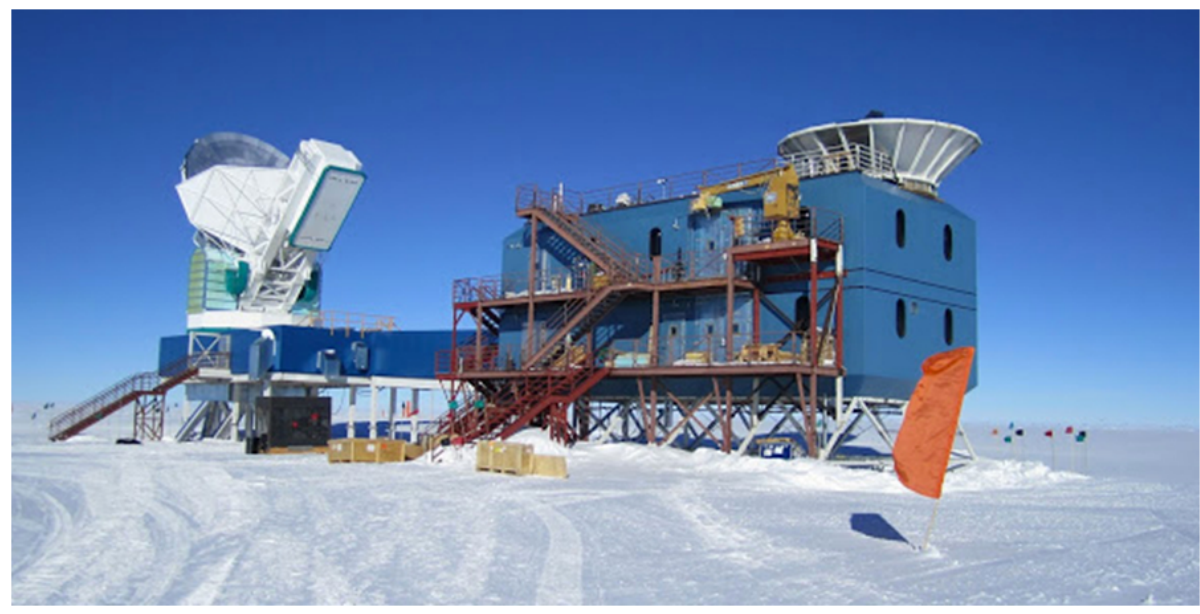

Figure 44: BICEP experiment at the South Pole (https://physicsforme.com/tag/bicep2/). 
In particular, if Inflation happened immediately after the Big Bang, it would have produced turbulence in the structure of space-time itself-gravitational waves like the kind LIGO detected recently. While these waves would be too weak for LIGO to see, they would twist the orientation of the light, which is known as polarization.

A few years ago the collaboration of the BICEP2 experiment claimed the detection of E-mode (Crites et al., 2015) and B-mode polarization of the CMB at at $7.0 \sigma$ significance (Ade et al., 2015). If B-mode polarization would be confirmed, the inflationary model of the Universe would be definitively confirmed. However, big discoveries need big confirmations. For a robust detection of B-modes, independent measurements and precise measurements of polarized foregrounds are mandatory.

Indeed, a key element to the primordial interpretation advanced by the BICEP2 team was excluding an explanation based on polarized thermal dust emission from our galaxy (Bucher, 2015a,b). An independent analysis casted doubt on the BICEP2 claim (Flauger, Hill \& Spergel, 2014). In September 2014 the Planck team published a paper on the level of polarized dust emission measured across the whole sky, and in particular in the BICEP2 field (Planck Collaboration, 2014). This work also extrapolated the polarized dust signal seen in the Planck $353 \mathrm{GHz}$ map (a frequency in the Wien tail of the CMB blackbody where dust dominates) down to $150 \mathrm{GHz}$ and reached the conclusion that the BICEP B mode signal could be entirely explained by polarized dust emission although a primordial B mode contribution could not be ruled out.

However, the theory of inflation is criticized by Ijjas, Steinhardt \& Loeb (2013) after Planck2013 results. They suggest that the origin of the Universe is not the Big Bang, but could be a "bouncing" Universe that does not need the inflation. Membrane-Universes that clashed endlessly could be a "plausible" alternative model for the Universe (Erickson et al., 2007; Steinhardt, Turok \& Starkman, 2008). Cyclic models of the universe have the advantage of avoiding initial conditions problems related to postulating any sort of beginning in time (Ijjas, 2018; Ijjas \& Steinhardt, 2018).

For all these reasons is even more important to find an experimental proof of the Inflation.

\subsection{Square Kilometre Array}

From the SKA Global Headquarters, Friday 19 December 2019 the good news has been reported: An independent panel of external reviewers from major astronomy projects has given the SKA's overall design, costing \& planning the nod, clearing the way for the preparation of the SKA construction proposal.

The Square Kilometre Array (SKA) is a planned large radio interferometer designed to operate over a wide range of frequencies, and with an order of magnitude greater sensitivity and survey speed than any current radio telescope. The SKA will address many important topics in astronomy, ranging from planet formation to distant galaxies (Weltman et al., 2018). In their work, they consider the perspective of the SKA as a facility for studying physics. They review four areas in which the SKA is expected to make major contributions to our understanding of fundamental physics: cosmic dawn and reionisation; gravity and gravitational radiation; cosmology and dark energy; and dark matter and astroparticle physics. These discussions demonstrate that the SKA will be a spectacular physics machine, which will provide many new breakthroughs and novel insights on matter, energy and spacetime. 

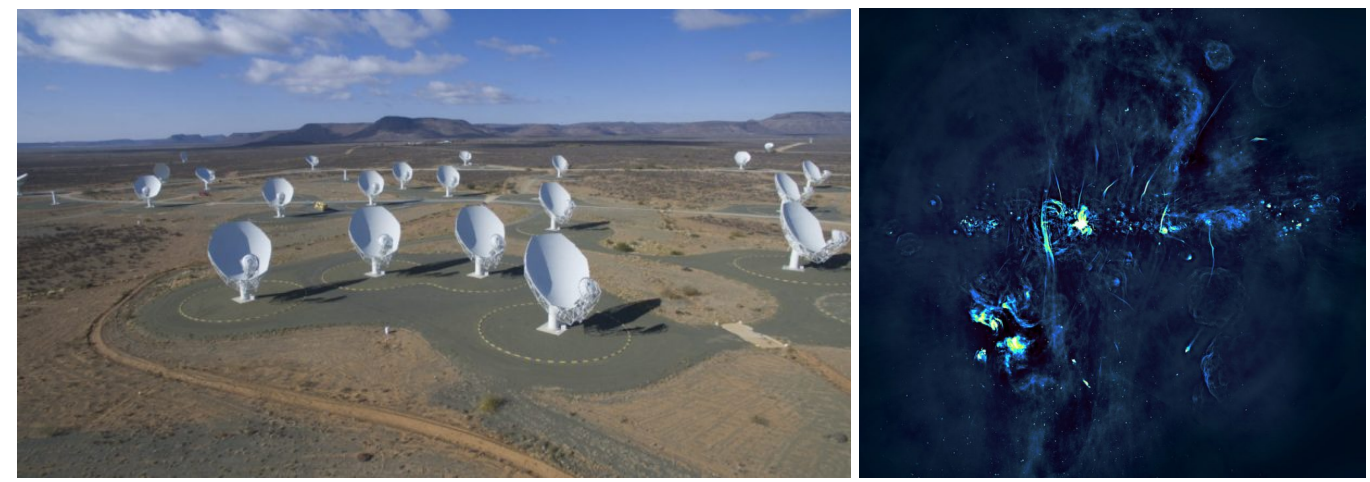

Figure 45: Left panel: South Africa's MeerKAT radiotelescope is the prototype for the SKA. Credit: South African Radio Astronomy Observatory. Right panel: The stunning radio image obtained by MeerKAT shows the central portions of the Milky Way galaxy. Its plane, marked by a series of bright features, runs horizontally through the image, while the newly discovered radio bubbles extend vertically above and below. (Credit: SARAO. Adapted from results published in Heywood et al., 2019.)

Figure 45 (left panel) shows the South Africa's MeerKAT radiotelescope which is the prototype for the SKA. Figure 45 (right panel) shows the impressive result coming from MeerKAT radiotelescope about the central portion of our Galaxy (Credit: SARAO - adapted from Heywood et al. (2019).

Of course the list of big experiments is far to be complete, but it is enough to show to the reader the efforts that the international scientific community are facing both for determining the frontier scientific tests to validate the current theories and for the difficulties in providing sufficient budgets for their realization.

\section{Conclusions}

In this review paper we have discussed several arguments that in our opinion are fundamental for the comprehension of the physics of our Universe. We have emphasized with some examples the use of wisdom in physics.

It is important the Great example of synergy between Astrophysics and History we discussed for demonstrating that Sedov's formula for determining the age of SNRs can be revisited, thanks to the recalibration of the age of the SNR Vela Jr (Aschenbach, 2016) experimentally supported by a historical document.

We discussed about the small and big space- and ground-based experiments that provide measurements necessary for the advancement of the knowledge of the physics of our Universe. Thanks to these results we discussed the present situation about the problems resolved and those still open, far from the completeness due to our limited knowledge.

As we discussed in the introduction, we can state that the Universe is interconnected in all its components: from cosmic network, to clusters of galaxies, to galaxies, to stars, to planets, to living beings, up to the simple bacterium. Therefore even every manifestation of life on our planet is subject to interconnection with all the surrounding environment. We can affirm that the 
whole Universe is a vital whole interconnected with more or less strong links between the various components, but that certainly exist.

Finally we can conclude with Fig. 46 that clearly explain all the mysteries of our Universe (Giovannelli, 2000), or if you prefer the same attempt written in Fig. 47. People who are able to read these sentences can understand that "The truth is written in the book of the Nature. We must learn to read this book".

$$
\begin{aligned}
& \text { 自然以山教科書には } \\
& \text { 真央がある。 } \\
& \text { ただ我々は } \\
& \text { 読み方を学ば } \\
& \text { なければならない }
\end{aligned}
$$

Figure 46: Understanding our Universe (Giovannelli, 2000).

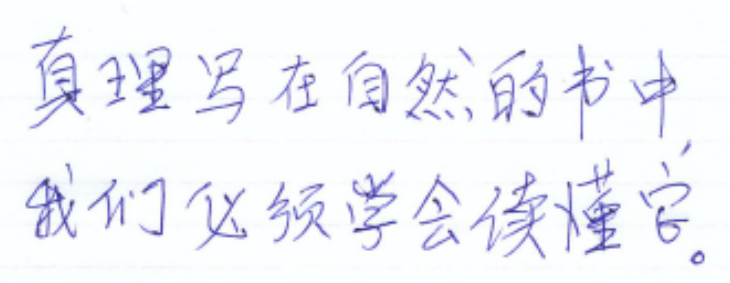

Figure 47: Understanding our Universe.

The experiments provide the basic alphabet, immersed in an apparently chaotic soup, but necessary to understand the nature. From that soup we must extract words and phrases to compose the book of the nature. In other words, the data coming from the experiments constitute the basic alphabet that we use for constructing models that attempt to describe the nature. But we have a lot of models for interpreting the experimental data by the light of science. Depending on the hypotheses the results could run against the experiments. Then, in order to be acceptable, models can take into account and justify ALL the available data.

The same concept was expressed in much more incisive terms by Richard Phillips Feynman - Nobel laureate in Physics in 1965 - also known as The Great Explainer: It doesn't matter how beautiful your theory is, it doesn't matter how smart you are. If it doesn't agree with experiment, it's wrong. 


\section{Acknowledgments}

This research has made use of "The NASA's Astrophysics Data System".

\section{References}

[1] Aad, G. (The ATLAS Collaboration): 2012, PhL B 718, 369-390.

[2] Aaij, R. et al. (LHCb Collaboration): 2015, PRL 115, Issue 7, id. 072001, 48 pp.

[3] Abbott, B.P. et al.: 2016a, PhRvL 116, 061102.

[4] Abbott, B.P. et al.: 2016b, PhRvL 116,1103.

[5] Abbott, B.P. et al.: 2016c, Living Rev. Relativity 19, Issue 1, article id. 1, 39 pp.

[6] Abbott, B.P. et al.: 2017a, PhRvL 119, Issue 16, id.161101, 18 pp.

[7] Abbott, B.P. et al.: 2017b, ApJL 848, Issue 2, article id. L12, 59 pp.

[8] Abbott, B.P. et al. (The LIGO Scientific Collaboration and The Virgo Collaboration, The 1M2H Collaboration, The Dark Energy Camera GW-EM Collaboration and the DES Collaboration, The DLT40 Collaboration, The Las Cumbres Observatory Collaboration, The VINROUGE Collaboration $\&$ The MASTER Collaboration): 2017c, Nature 551, 85-88.

[9] Abbott, B.P. et al. (LIGO Scientific Collaboration and Virgo Collaboration): 2019, PhR X 9, Issue 3, id.031040.

[10] Abdo, A.A., Ackermann, M., Ajello, M., Allafort, A., Atwood, W.B. et al.: 2010, ApJ, 723, Issue 2, 1082-1096.

[11] Abe, K., Abgrall, N., Aihara, H., Ajima, Y., Albert, J.B. et al.: 2011, Nucl. Instr. \& Meth. in Phys. Res. A, 659, Issue 1, 106-135.

[12] Abe, K., J. Adam, J., Aihara, H., Akiri, T., Andreopoulos, C. et al.: 2015, arXiv:1409.7469v2 [hep-ex] 10 Feb 2015.

[13] Acharya, B.F., Agudo, I., Al Samaral, I., Alfaro, R., Alfaro, J. et al. (The CTA Consortium): 2019, Science with the Cherenkov Telescope Array. Edited by CTA Consortium. Published by World Scientific Publishing Co. Pte. Ltd. - Also in arXiv:1709.07997v2.

[14] Acquafredda, R., Adam, T., Agafonova, N., Alvarez Sanchez, P., Ambrosio, M. et al.: 2009, J. Instr. 04, Issue 04, 04018.

[15] Ade, P.A.R. et al. (BICEP Collaboration): 2015, PhRvL 114, Issue 10, id. 101301.

[16] Adelberger, E.G., Austin, S.M., Bahcall, J.N., Balantekin, A.B., Bogaert, G. et al.: 1998, Rev. Mod. Phys., 70, 1265-1291.

[17] Adelberger, E.G., García, A., Robertson, R.G. Hamish, Snover, K.A., Balantekin, A.B. et al.: 2011, Rev. Mod. Phys., 83, Issue 1, 195-246.

[18] Albert, J. \& Magic Collaboration: 2008, Science 320, Issue 5884, 1752-1754.

[19] Amaro-Seoane, P., Audley, H., Babak, S., Baker, J., Barausse, E. et al.: 2017, arXiv:1702.00786.

[20] Amati, L., O’Brien, P., Götz, D., Bozzo, E., Tenzer, C. et al.: 2018, Adv. Spa. Res. 62, Issue 1, 191-244.

[21] Anders, M., Trezzi, D., Bellini, A., Aliotta, M., Bemmerer, D. et al.: 2013, EPJA, 49, id.28. 
[22] Anders, M., Trezzi, D., Menegazzo, R., Aliotta, M., Bellini, A. et al. (LUNA Collaboration): 2014, Phys. Rev. Lett. 113, Issue 4, id.042501.

[23] Aschenbach, B.: 1998, Nature 396, Issue 6707, 141-142.

[24] Aschenbach, B.: 2016, in Frontier Research in Astrophysics II. Online at https://pos.sissa.it/cgi-bin/reader/conf.cgi?confid=269, id.36.

[25] Auriemma, G., Costa, E., Giovanneli, F., Medici, G., Ubertini, P.: 1975, talk presented at the workshop on Italian Extragalactic Astronomy on April 3, 1975. Internal Report N. 26, 1975 of the LAS (Laboratorio di Astrofisica, CNR).

[26] Baade, W., Zwicky, F.: 1934a, Phys. Rev. 45, 138.

[27] Baade, W., Zwicky, F.: 1934b, Phys. Rev. 46, 76-77.

[28] Bañados, E., Novak, M., Neeleman, M., Walter, F., Decarli, R. et al.: 2019, ApJ 881, Issue 1, article id. L23, 6 pp.

[29] Bandura, K., Addison, G.E., Amiri, M., Bond, J.R., Campbell-Wilson, D. et al.: 2014, Proc. SPIE 9145, id. 914522, 20 pp.

[30] Barone, F., Di Fiore, L., Milano, L., Russo, G.: 1992, General Relativity and Gravitation, 24, No. 3, 323-341.

[31] Bennett, C.L., Larson, D., Weiland, J.L., Jarosik, N., Hinshaw, G.: 2013, ApJS 208, Issue 2, article id. 20,54 pp.

[32] Bennett, C.L., Larson, D., Weiland, J.L., Hinshaw, G.: 2014, ApJ 794, Issue 2, article id. 135, 8 pp.

[33] Berger, E., Fong, W., Chornock, R.: 2013, ApJL 774, Issue 2, article id. L23, 4 pp.

[34] Bernardini, M.G.: 2015, J.HE Astrophys. 7, 64-72.

[35] de Bernardis, P., Ade, P.A.R., Bock, J.J., Bond, J.R., Borrill, J. et al.: 2000, Nature 404, Issue 6781, 955-959.

[36] de Bernardis, P.: 2014, talk at the Mondello Workshop Frontier Research in Astrophysics - I.

[37] Bisikalo, D.V., Zhilkin, A.G.: 2015, Acta Polytechnica CTU Proc. 2(1), 60-65.

[38] Bisnovatyi-Kogan, G.S.: 2002, in Black Hole Astrophysics 2002, Proc. of the Sixth APCTP Winter Schoo, Pohang, Korea, pp. 187-206.

[39] Bisnovatyi-Kogan, G.S., Giovannelli, F.: 2017, A\&A 599, id. A55, 7 pp.

[40] Blandford, R.D., Kochanek, C.S.: 2004, in Dark Matter in the Universe (Second Edition), J. Bahcall et al. (Eds.), World Scientific Publishing Co. Pte. Ltd., ISBN 9789812567185, pp. 103-158.

[41] Bloom, J., Castro-Tirado, A.J., Hanlon, L., Kotani, T. (Eds.): 2010, I Workshop on Robotic Autonomous Observatories, Advances in Astronomy, Vol. 2010.

[42] Bouvier, J.: 1990, AJ 99, 946-964.

Nature 555, Issue 7694, 67-70.

[43] Broggini, C., Bemmerer, D., Guglielmetti, A., Menegazzo, R.: 2010, Annu. Rev. Nucl. Part. Sci. 60, 53-73.

[44] Broggini, C., Bemmerer, D., Caciolli, A., Trezzi, D.: 2018, Progr. in Part. and Nucl. Phys. 98, 55-84.

[45] Bromm, V., Loeb, A.: 2002, ApJ 575, Issue 1, 111-116. 
[46] Bruno, C.G., Scott, D.A., Aliotta, M., Formicola, A., Best, A. et al. (LUNA Collaboration): 2016, Phys. Rev. Lett. 117, Issue 14, id.142502.

[47] Bucher, M. (on behalf of the PLANCK Collaboration): 2015a, Nucl and Part. Phys. Proc. 267-269, p. 245-253.

[48] Bucher, M. (on behalf of the PLANCK Collaboration): 2015b, Int. J. Mod. Phys. D, Volume 24, Issue 2, id. 1530004-303.

[49] Buckley, D.A.H.: 2015, talk at the Palermo Workshop 2015 on The Golden Age of Cataclysmic Variables and Related Objects - III.

[50] Buckley, D.A.H., Meintjes, P.J., Potter, S.B., Marsh, T.R., Gänsicke, B.T.: 2017, Nature Astronomy 1, id. 0029.

[51] Buson, S. (Fermi-LAT Collaboration): 2014, in Frobtier Research in Astrophysics, Franco Giovannelli \& Lola Sabau-Graziati (Eds.), http://pos.sissa.it/cgi-bin/reader/conf.cgi?confid=237, id. 7.

[52] Caballero-García, M.D., Pandey, S.B., Hiriart, D. \& Castro-Tirado, A.J. (Eds.): 2016, IV Workshop on Robotic Autonomous Observatories, RMxAC, Vol. 48.

[53] Camilo, F., Ransom, S.M., Halpern, J.P., Reynolds, J., Helfand, D.J. et al.: 2006, Nature, 442, Issue 7105, 892-895.

[54] Cardini, A.: 2015, Interview of Alessandro Cardini, P.I. of LHCb, 14th July 2015.

[55] Castro Cerón, J.M.: 2011, talk at the Frascati Workshop 2011 on "Multifrequency Behaviour of High Energy Cosmic Sources".

[56] Castro-Tirado, A.J.: 2008, in 3rd Symposium of the Astrophysics Group of the Spanish Royal Physical Society (RSEF), A. Ulla \& M. Manteiga (Eds.), Lecture Notes and Essays in Astrophysics 3, 131.

[57] Castro-Tirado, A.J.: 2010a, Adv. Astron. Vol. 2010, Article ID 570489, 8 pages.

[58] Castro-Tirado, A.J.: 2010b, Adv. Astron. Vol. 2010, Article ID 824731, 1 page.

[59] Cautun, M., Benitez-Llambay, A., Deason, A.J., Frenk, C.S., Fattahi, A. et al.: 2019, arXiv:1911.04557.

[60] Cavanna, F. (on behalf of the LUNA collaboration): 2018, in International Symposium on t' Capture Gamma-Ray Spectroscopy and Related Topics (CGS16), Sun, Yang (Ed.), EPJ Web of Conferences, Volume 178, id.01007.

[61] Cavanna, F., Depalo, R., Aliotta, M., Anders, M., Bemmerer, D. et al. (LUNA Collaboration): 2015, Phys. Rev. Lett. 115, Issue 25, id.252501.

[62] Ciardi, B., Loeb, A.: 2000, ApJ 540, Issue 2, 687-696.

[63] Class for Physics of the Royal Swedish Academy of Sciences: 2011, The accelerating Universe: Scientific Background on the Nobel Prize in Physics 2011.

[64] Cooray, A.: 2016, R. Soc. open sci. 3: 150555. http://dx.doi.org/10.1098/rsos.150555.

[65] Coppi, P.S., Aharonian, F.A.: 1997, ApJ, 487, Issue 1, L9-L12.

[66] Costa, E., Frontera, F., Heise, J., Feroci, M., in’t Zand, J. et al.: 1997, Nature 387, Issue 6635, 783-785.

[67] Costamante, L.: 2012, in Multifrequency Behaviour of High Energy Cosmic Sources, F. Giovannelli \& L. Sabau-Graziati (Eds.), Mem. SAIt., 83, 138-145. 
[68] Crites, A.T., Henning, J.W., Ade, P.A.R., Aird, K.A., Austermann, J.E. et al.: 2015, ApJ 805, 36, 18 pp.

[69] Cucchiara, A., Levan, A.J., Fox, D.B., Tanvir, N.R., Ukwatta, T.N. et al.: 2011, ApJ, 736, Issue 1, article id. 7, $12 \mathrm{pp}$.

[70] Cumani, P., Galper, A.M., Bonvicini, V., Topchiev, N.P., Adriani, O. et al.: 2015, arXiv:1502.02976.

[71] Dado, S., Dar, A., De Rújula, A.: 2009, ApJ 696, Issue 1, 994-1020.

[72] Dado, S., Dar, A.: 2013a, ApJ 775, Issue 1, article id. 16, 7 pp.

[73] Dado, S., Dar, A.: 2013b, A\&A 558, id.A115, 7 pp.

[74] Dado, S., Dar, A.: 2016, PhRvD 94, Issue 6, id.063007.

[75] Dai, X., Kochanek, C.S.: 2005, ApJ 625, Issue 2, 633-642.

[76] Damon, E., Eisler, D., Rasolt, D., Shaw, A., Story, K. et al.: 2004, Proc. of the Fall 2004 Astronomy 233 Symposium on Measurements of the Hubble Constant, D.B. Campbell \& J. Deneva (Eds), offered by the Cornell University Astronomy Department and the College of Arts and Sciences under the John S. Knight Institute Sophomore Seminar Program, 61 pp.

[77] Dar, A.: 1997, in Very High Energy Phenomena in the Universe Morion Workshop. ISBN 2-86332-217-6 Editions Frontieres, Y. Giraud-Heraud \& J. Tran Thanh Van (Eds.), p.69. (arXiv:astro-ph/9704187v2).

[78] Dar, A.: 2003, in Frontier Objects in Astrophysics and Particle Physics, F. Giovannelli \& G. Mannocchi (Eds.), Italian Physical Society 85, 253-265.

[79] Dar, A.: 2006, ChJA\&A Supp. 6, Issue S1, 323-329.

[80] Dar, A.: 2017, talk at the Frascati Workshop 2017 Multifrequency Behaviour of High Energy Cosmic Sources - XIII.

[81] Dar, A., De Rújula, A.: 2000, arXiv:astro-ph/0008474.

[82] D’Avanzo, P.: 2015, J. HE Astrophys. 7, 73-80.

[83] De Angelis, A., Tatischeff, V., Tavani, M., Oberlack, U., Grenier, I. et al.: 2017, Exp. Astron. 44, Issue $1,25-82$.

[84] Depalo, R., Cavanna, F., Aliotta, M., Anders, M., Bemmerer, D. et al. (LUNA Collaboration): 2016, Phys. Rev. C. 94, 055804.

[85] De Pasquale, M., Page, M.J., Kann, D.A., Oates, S.R., Schulze, S. et al.: 2016, MNRAS 462, Issue 1, 1111-1122.

[86] Di Leva, A., Scott, D.A., Caciolli, A., Formicola, A., Strieder, F. et al. (LUNA Collaboration): 2014, Phys. Rev. C, 89, Issue 1, id.015803 and erratum Phys. Rev C, 90, Issue 1, id.019902.

[87] Dopita, M.A., Krauss, L.M., Sutherland, R.S., Kobayashi, C., Lineweaver, C.H.: 2011, Astrophys. Space Sci., 335, Issue 2, 345-352.

[] Duncan, R.C., Thompson, C., 1992, ApJ 392, L9-L13.

[88] Durrer, R.: 2015, Classical and Quantum Gravity, 32, Issue 12, article id. 124007.

[89] Enoto, T.: 2018, in Quarks and Compact Stars 2017 (QCS2017), JPS Conf. Proc., id.011046, 7 pp.

[90] Erickson, J.K., Gratton, S., Steinhardt, P.J., Turok, N.: 2007, PhRvD 75, Issue 12, id. 123507. 
[91] Evans, P.A., Beardmore, A.P., Page, K.L., Osborne, J.P., O’Brien, P.T. et al.: 2009, MNRAS 397, 1177-1201.

[92] Fan, X., Strauss, M.A., Schneider, D.P., Becker, R.H., White, R.L. et al.: 2003, AJ 125, Issue 4, 1649-1659.

[93] Ferrario, L., Wickramasinghe, D.: 2006, MNRAS 367, Issue 3, 1323-1328.

[94] Fishbach, M., Gray, R., Magaña Hernandez, I., Qi, H., Sur, A. et al.: 2019, ApJ 871, Issue 1, article id. L13, 10 pp.

[95] Flauger, R., Hill, J.C., Spergel, D.N.: 2014, JCAP Issue 08, article id. 039. (arXiv:1405.7351).

[96] Freedman, W.L., Madore, B.F.: 2010, ARA\&A 48, 673-710.

[97] Fumagalli, M., Patel, S.G., Franx, M., Brammer, G., van Dokkum, P. et al.: 2012, APJL, 757, Issue 2, article id. L22, 6 pp.

[98] Galper, A.M., Topchiev, N.P., Yurkin, Yu.T.: 2018, ARep 62, Issue 12, 882-889.

[99] Gardner, J.P.: 2009, in AKARI, a Light to Illuminate the Misty Universe, Takashi Onaka, Glenn J. White, Takao Nakagawa \& Issei Yamamura (Eds.), ASP Conf. Ser. 418, 365-373.

[100] Gardner, J.P., Mather, J.C., Clampin, M., Doyon, R., Greenhouse, M.A. et al.: 2006, SSRv 123, Issue 4, 485-606.

[101] Gehrels, N., Cannizzo, J.K.: 2015, J. HE Astrophys. 7, 2-11.

[102] Gell-Mann, M.: 1964, PhL, 8, Issue 3, 214-215.

[103] Ghirlanda, G., Bernardini, M.G., Calderone, G., D’Avanzo, P.: 2015, J. HE Astrophys. 7, 81-89.

[104] Ghosh, S.K.: 2009, in Frontier Objects in Astrophysics and Particle Physics, F. Giovannelli \& G. Mannocchi, (Eds.), SIF, Bologna, Italy, 98, 243-254.

[105] Giacconi, R., Gursky, H., Paolini, F.R., Rossi, B.B.: 1962, PhRvL 9, 439-443.

[106] Giallongo, E., D’Odorico, S., Fontana, A., McMahon, R.G., Savaglio, S. et al.: 1994, ApJ, 425, no. 1, L1-L4.

[107] Gianotti, F.: 2012, 4th July, talk at CERN.

[108] Gilmozzi, R.: 2008, Extremely Large Telescopes: Which Wavelengths?, Retirement Symposium for Arne Ardeberg. Edited by Andersen, Torben E. Proceedings of the SPIE, Volume 6986, article id. 698604, 8 pp.

[109] Gilmozzi, R.: 2009, The Messenger 136, 2-7.

[110] Gilmozzi, R.: 2013, talk at the Palermo Workshop 2013 on The Golden Age of Cataclysmic Variables and Related Objects - II.

[111] Gilmozzi, R., Spyromilio, J.: 2007, The Messenger 127, 11-19.

[112] Gilmozzi, R., Spyromilio, J.: 2009, in Science with the VLT in the ELT Era, Ap\&SS Proc. Springer Netherlands, pp. 217-223.

[113] Gilmozzi, R., Kissler-Patig, M.: 2011, The Messenger 143, 25-25.

[114] Giovannelli, F.: 1994, SSRv 69, Issue 1-2, 1-138.

[115] Giovannelli, F.: 2000, in The Evolution of The Milky Way, F. Matteucci \& F. Giovannelli (Eds.), Kluwer Academic Publishers, pp. 619-620. 
[116] Giovannelli, F.: 2001, The bridge between the big bang and biology: stars, planetary systems, atmospheres, volcanoes: their link to life, Franco Giovannelli (Ed.), Consiglio Nazionale delle Ricerche (CNR): President's Bureau of the CNR, Mario Apice (General Coordinator), pp. 1-440.

[117] Giovannelli, F.: 2010, in Multifrequency Behaviour of High Energy Cosmic Sources, F. Giovannelli \& L. Sabau-Graziati (Eds.), Mem. SAIt 81, 510-513.

[118] Giovannelli, F.: 2016, in High Energy Astrophysics in Southern Africa (HEASA 2016). Online at http://pos.sissa.it/cgi-bin/reader/conf.cgi?confid=275, id.31.

[119] Giovannelli, F., Errico, L., Vittone, A.A., Rossi, C.: 1991, A\&AS 87, 89-95.

[120] Giovannelli, F., Sabau Graziati, L., La Padula, C., Errico, L., Frutti, M. et al.: 1993, A\&AS 97, 395-397.

[121] Giovannelli, F., Sabau-Graziati, L., La Padula, C.D., Isern, J., Bravo, E., Garcia-Berro, E., Gómez-Gomar, J., Guerrero, J., Hernans, M., José, J.: 1999a, Mem. SAIt. 70, 1317-1346.

[122] Giovannelli, F., La Padula, C.D., Sabau-Graziati, L., Talavera, A., Angulo, M., Isern, J. et al.: 1999b, in Frontier Objects in Astrophysics and Particle Physics, F. Giovannelli \& G. Mannocchi (Eds.), Italian Physical Society 65, 623-644.

[123] Giovannelli, F., Sabau-Graziati, L., Isern, J., La Padula, C.D., Angulo, M. et al.: 2001, in Frontier Objects in Astrophysics and Particle Physics, F. Giovannelli \& G. Mannocchi (Eds.), Italian Physical Society 73, 565-579.

[124] Giovannelli, F., Sabau-Graziati, L., Isern, J., La Padula, C.D., Angúlo, M. et al.: 2002a, SIXE (Spanish Italian X-ray Experiment) - Feasibility Study Report. (PNIE-CICYT Report (ESP97-1784-E grant), IASF (Istituto di Astrofisica Spaziale e Fisica Cosmica, Roma), Technical Report No. 4, December 2002.

[125] Giovannelli, F., Sabau-Graziati, L., Isern, J., La Padula, C.D., Angulo, M. et al.: 2002b, Mem. SAIt $73,402-417$.

[126] Giovannelli, F., Sabau-Graziati, L.: 2004, SSR, 112, 1-443 (GSG2004).

[127] Giovannelli, F., Sabau-Graziati, L.: 2006, Chinese J. A\&A Suppl. 6, Issue S1, p. 1-28.

[128] Giovannelli, F., Sabau-Graziati, L.: 2011, Acta Polytechnica Vol. 51, No. 2., p. 21.

[129] Giovannelli, F., Bisnovatyi-Kogan, G.S., Klepnev, A.S.: 2013, A\&A 560, id.A1, 11 pp (GBK13).

[130] Giovannelli, F., Sabau-Graziati, L.: 2014, in Multifrequency Behaviour of High Energy Cosmic Sources - X, Acta Polytechnica CTU Proceedings 1(1), p.1-12.

[131] Giovannelli, F., Bisnovatyi-Kogan, G.S., Bruni, I., Corfini, G., Martinelli, F., Rossi, C.: 2015, AcA $65,107-116$.

[132] Giovannelli, F., Sabau-Graziati, L. (Eds.): 2015, The Golden Age of Cataclysmic Variables and Related Objects - III, Online at http://pos.sissa.it/cgi-bin/reader/conf.cgi?confid=255, id. 1.

[133] Giovannelli, F., Sabau-Graziati, L.: 2016a, in Accretion Processes in Cosmic Sources, online at http://pos.sissa.it/cgi-bin/reader/conf.cgi?confid=288, id.1.

[134] Giovannelli, F., Sabau-Graziati, L. (Eds.): 2016b, Accretion Processes in Cosmic Sources: Young Stellar Objects, Cataclysmic Variables and Related Objects, X-ray Binary Systems, Active Galactic Nuclei - I, Online at http://pos.sissa.it/cgi-bin/reader/conf.cgi?confid=288. 
[135] Giovannelli, F., Sabau-Graziati, L.: 2016c, in Frontier Research in Astrophysics II, Online at https://pos.sissa.it/cgi-bin/reader/conf.cgi?confid=269, id. 1 .

[136] Gnedin, N.Y.: 2000, ApJ, 535, 530-554.

[137] Gnedin, N.Y., Glover, S.G.O., Klessen, R.S., Springel, V. (Eds.): 2015, Star Formation in Galaxy Evolution: Connecting Numerical Models to Reality, Springer, ISBN 978-3-662-47889-9.

[138] Guseinov, O.Kh., Zel'dovich, Ya.B.: 1966, Sov. Astr. 10, 251-253.

[139] Gustavino, C.: 2007, in Frontier Objects in Astrophysics and Particle Physics, F. Giovannelli \& G. Mannocchi (Eds.), Italian Physical Society, Ed. Compositori, Bologna, Italy, 93, 191-204.

[140] Gustavino, C.: 2009, in Frontier Objects in Astrophysics and Particle Physics, F. Giovannelli \& G. Mannocchi (Eds.), Italian Physical Society, Ed. Compositori, Bologna, Italy, 98, 77-90.

[141] Gustavino, C.: 2011, in Frontier Objects in Astrophysics and Particle Physics, F. Giovannelli \& G. Mannocchi (Eds.), Italian Physical Society, Ed. Compositori, Bologna, Italy, 103, 657.

[142] Gustavino, C.: 2012, in Nuclei in the Cosmos (NIC XII), http://pos.sissa.it/cgi-bin/reader/conf.cgi?confid=146, id.74

[143] Gustavino, C.: 2013, AcPol 53, 534-537.

[144] Gustavino, C. (on behalf of the LUNA collaboration): 2019, in RICAP18, 7th Roma International Conference on Astroparticle Physics, Edited De Vincenzi, M., Capone, A. \& Morselli, A. (Eds.), EPJ Web of Conferences, Volume 209, id.01043.

[145] Guziy, S., Pandey, S.B., Tello, J.C. \& Castro-Tirado, A.J. (Eds.): 2012, II Workshop on Robotic Autonomous Observatories, Astron. Soc. of India Conf. Ser., Vol. 7.

[146] Hamuy, M., Pignata, G., Maza, J., Clocchiatti, A., Anderson, J. et al.: 2012, Mem. S.A.It. Vol. 83, 388-392.

[147] Harding, A.K.: 2013, Front. Phys., 8, Issue 6, 679-692.

[148] Haridasu, B.S., Lukovic, V.V., D’Agostino, R., Vittorio, N.: 2017, A\&A 600, id. L1, 5 pp.

[149] Hartwig, T., Volonteri, M., Bromm, V., Klessen, R.S., Barausse, E. et al.: 2016, MNRAS Lett. 460, Issue 1, L74-L78.

[150] Henry, R.C., Murthy, J., Overduin, J., Tyler, J.: 2015, ApJ 798, Issue 1, article id. 14, 25 pp.

[151] Heywood, I., Camilo, F., Cotton, W.D., Yusef-Zadeh, F., Abbott, T.D. et al.: 2019, Nature 573, Issue $7773,235-237$.

[152] Hillas, A.M.: 1984, ARA\&A 22, 425-444.

[153] Hofmann, W. (for the CTA Consortium): 2017, The Messenger 168, 21-25.

[154] Hopkins, A,M., Beacom, J.F., 2006, ApJ, 651, 142-154 - Erratum: 2008, ApJ 682, 1486.

[155] Huchra, J.: 2008, https://www.cfa.harvard.edu/ dfabricant/huchra/hubble/

[156] Huchra, J.: 2010, https://www.cfa.harvard.edu/ dfabricant/huchra/hubble.plot.dat

[157] Hudec, R.: 2017, in Multifrequency behaviour of High Energy Cosmic Sources - XII, Online at https://pos.sissa.it/cgi-bin/reader/conf.cgi?confid=306, id.83.

[158] Hulleman, F., van Kerkwijk, M.H., Kulkarni, S.R.: 2000, Nature 408, Issue 6813, 689-692. 
[159] Hurley, K.: 2007, talk at the Frascati Workshop 2007 on Multifrequency Behaviour of High Energy Cosmic Sorces - VII.

[160] Hurley, K.: 2008, ChJA\&A Suppl. 8, 202-212.

[161] Ibrahim, A.I., Markwardt, C.B., Swank, J.H., Ransom, S., Roberts, M. et al.: 2004, ApJ 609, Issue 1, L21-L24.

[162] Ijjas, A.: 2018, Classical and Quantum Gravity Vol. 35, Issue 7, article id. 075010.

[163] Ijjas, A., Steinhardt, P.J., Loeb, A.: 2013, PhLB 723, Issue 4-5, 261-266.

[164] Ijjas, A., Steinhardt, P.J.: 2018, Classical and Quantum Gravity, Vol. 35, Issue 13, article id. 135004.

[165] Illarionov, A.F., Sunyaev, R.A.: 1975, A\&A 39, 185.

[166] Incandela, J.: 2012, UCSB/CERN, Talk on July 4, 2012.

[167] Isern, J., Giovannelli, F. (Principal Investigators), Angúlo, M., Bausells, J., Bravo, E., Cabestany, J., García-Berro, E., Gómez-Gomar, J., Hernanz, M., La Padula, C.D., Sabau-Graziati, L., Talavera, A. (Co-Investigators): 1999a, SIXE (Spanish Italian X-ray Experiment) - Feasibility Study Report, PNIE-CICYT Report (ESP97-1784-E grant).

[168] Isern, J., Bravo, E., Gómez-Gomar, J., Hernanz, M., García-Berro, E., Giovannelli, F., La Padula, C.D., Sabau, L. et al.: 1999b, AIPC 499, 110.

[169] Jakobs, K., Seez, C.: 2015, Scholarpedia 10(9):32413.

[170] Jiang, L., Egami, E., Kashikawa, N., Walth, G., Matsuda, Y. et al.: 2011, ApJ 743, Issue 1, article id. $65,10 \mathrm{pp}$.

[171] Kaplinghat, M., Chu, M., Haiman, Z., Holder, Gilbert P. et al.: 2003, ApJ 583, Issue 1, 24-32.

[172] Kashikawa, N., Shimasaku, K., Malkan, M.A., Doi, M., Matsuda, Y. et al.: 2006, ApJ 648, Issue 1, 7-22.

[173] Kashikawa, N.: 2007, in At the Edge of the Universe: Latest Results from the Deepest Astronomical Surveys, J. Afonso, H.C. Ferguson, B. Mobasher \& R. Norris (Eds.), ASP Conf. Series 380, 11-16.

[174] Kaspi, V.M.: 2010, PNAS 107, Issue 16, 7147-7152.

[175] Kaspi, V.M., Beloborodov, A.M.: 2017, ARA\$A 55, issue 1, 261-301.

[176] Keating, B.G., Ade, P.A.R., Bock, J.J., Hivon, E., Holzapfel, W.L. et al.: 2003a, SPIE 4843, $284-295$.

[177] Keating, B.G., O’Dell, C.W., Gundersen, J.O., Piccirillo, L., Stebor, N.C., Timbie, P.T.: 2003b, ApJS 144, Issue 1, 1-20.

[178] Kennicutt, R.C., Jr.: 1998, ARA\&A, 36, 189-232.

[179] Kennicutt, R.C., Evans, N.J.: 2012, ARA\&A, 50, 531-608.

[180] Klebesadel, R.W., Strong, I.B., Olson, R.A.: 1973, ApJ 182, L85-L88.

[181] Kitamoto, S., Enoto, T., Safi-Harb, S., Pottschmidt, K., Ferrigno, C. et al.: 2014, arXiv:1412.1165, $40 \mathrm{pp}$.

[182] Knox, L.: 2003, New Astr. Rev. 47, Issue 11-12, 883-886.

[183] Kochanek, C.S.: 2003, in Hubble's Science Legacy: Future Optical/Ultraviolet Astronomy from Space, Kenneth R. Sembach, J. Chris Blades, Garth D. Illingworth and Robert C. Kennicutt, Jr. (Eds), ASPC 291, 245-252. 
[184] Kogut, A., Spergel, D.N., Barnes, C., Bennett, C.L., Halpern, M. et al.: 2003, ApJS 148, Issue 1, pp. 161-173.

[185] Komatsu, E., Smith, K.M., Dunkley, J., Bennett, C.L., Gold, B. et al.: 2011, ApJS, 192, Issue 2, article id. 18,47 pp.

[186] Kovetz, E.D., Viero, M.P., Lidz, A., Newburgh, L., Rahman, M. et al.: 2017, arXiv:1709.09066.

[187] Kumar, P., Zhang, B.: 2015, PhR 561, 1-109.

[188] Lamb, D.Q., Reichart, D.E.: 2000, ApJ 536, Issue 1, 1-18.

[189] Lamzin, S.A., Bisnovatyi-Kogan, G.S., Errico, L., Giovannelli, F., Katysheva, N.A. et al.: 1996, A\&A 306, 877-891.

[190] Lasota, J.-P.: 2001, New Astron. Rev. 45, 449-508.

[191] Liang, E-W., Zhang, B-B., Zhang, B.: 2007, ApJ 670, Issue 1, 565-583.

[192] Lipunov, V.M.: 1987, Ap\&SS 132, no. 1, 1-51.

[193] Lipunov, V.M.: 1995, in Frontier Objects in Astrophysics and Particle Physics, F. Giovannelli \& G. Mannocchi (Eds.), SIF, Bologna, Italy, 47, 61-76.

[194] Lipunov, V.M.: 2018, talk at the Mondello Workshop 2018 in Frontier Research in Astrophysics - III.

[195] Lipunov, V.M., Postnov, K.A.: 1988, Ap\&SS 145, no. 1, 1-45.

[196] Lipunov, V., Kornilov, V., Gorbovskoy, E., Shatskij, N., Kuvshinov, D. et al.: 2010, Advances in Astronomy, 2010, article id. 349171.

[197] Liske, J., Padovani, P., Kissler-Patig, M.: 2012, in Ground-based and Airborne Telescopes IV, Proc. SPIE 8444, article id. 84441I, 8 pp.

[198] Loeb, A., Barkana, R.: 2001, ARA\&A 39, 19-66.

[199] Luisi, P.L., Capra, F.: 2014, The systems View of Life, Cambridge Univ. Press, 510 pp.

[200] Madau, P., Dickinson, M.: 2014, ARA\&A, 52, 415-486.

[201] Marsh, T.R., Gänsicke, B.T., Hümmerich, S., Hambsch, F.-J., Bernhard, K. et al.: 2016, Nature 537, Issue $7620,374-377$.

[202] Martin, J., Rea, N., Torres, D.F., Papitto, A.: 2014, MNRAS 444, Issue 3, 2910-2924.

[203] Maselli, A., Melandri, A., Nava, L., Mundell, C.G., Kawai, N. et al.: 2014, Science 343, Issue 6166, 48-51.

[204] Matsuoka, Y., Onoue, M., Kashikawa, N., Iwasawa, K., Strauss, M.A. et al.: 2016, ApJ 828, Issue 1, article id. 26, 14 pp.

[205] Mazets, E.P., Golenetskij, S.V., Il'Inskij, V.N.: 1974, Pis'ma v ZhurnEhTF, Tom 19, p. 126-128.

[206] Mazets, E.P., Golenetskij, S.V., Guryan, Y.A.: 1979, Sov. Astr. Lett. 5, 343-344.

[207] McKee, C.F., Ostriker, E.C.: 2007, ARA\&A, 45, Issue 1, 565-687.

[208] McLaughlin, M.A., Stairs, I.H., Kaspi, V.M., Lorimer, D.R., Kramer, M.: 2003, ApJ 591, Issue 2, L135-L138.

[209] McPherson, A., Gilmozzi, R., Spyromilio, J., Kissler-Patig, M., Ramsay, S.: 2012, The Messenger $148,2-8$. 
[210] Meylan, G., Jetzer, P., North, P., Schneider, P., Kochanek, C.S., Wambsganss, J.: 2006, Gravitational lensing: strong, weak and micro, G. Meylan, P. Jetzer \& P. North (Eds.), Berlin: Springer, ISBN 3-540-30309-X, ISBN 978-3-540-30309-1, XIII + 552 pp. (Kochanek, C.S.: 2004, arXiv:astro-ph/0407232).

[211] Murray, S.: 2018, http://www.astronomy.com/news/2018/03/chime-begins-its-cosmic-search

[212] Nakar, E.: 2007, Phys. Rep. 442, 166-236.

[213] Nielsen, J.T., Guffanti, A., Sarkar, S. 2016, NatSR 6, id. 35596.

[214] Ogburn, R.W., IV, Ade, P.A.R., Aikin, R.W., Amiri, M., Benton, S.J., et al.: 2010, SPIE 7741, id. $77411 \mathrm{G}$.

[215] Olausen, S.A., Kaspi, V.M.: 2014a, ApJS 212, Issue 1, article id. 6, 22 pp.

[216] Olausen, S.A., Kaspi, V.M.: 2014b, Online Data Catalog: The McGill magnetar catalog http://www.physics.mcgill.ca/ pulsar/magnetar/table1apj.html

[217] Ono, Y., Ouchi, M., Mobasher, B., Dickinson, M., Penner, K. et al.: 2012, ApJ 744, Issue 2, article id. 83,13 pp.

[218] Orosz, J.A., Remillard, R.A., Bailyn, C.D., McClintock, J.E.: 1997, ApJL 478, Issue 2, L83-L86.

[219] Ota, K., Iye, M., Kashikawa, N., Shimasaku, K., Kobayashi, M. et al.: 2008, ApJ 677, Issue 1, 12-26.

[220] Ouchi, M., Mobasher, B., Shimasaku, K., Ferguson, H.C., Fall, S.M. et al.: 2009a, ApJ 706, Issue 2, 1136-1151.

[221] Ouchi, M., Ono, Y., Egami, E., Saito, T., Oguri, M. et al.: 2009b, ApJ 696, Issue 2, 1164-1175.

[222] Ouchi, M., Shimasaku, K., Furusawa, H., Saito, T., Yoshida, M. et al.: 2010, ApJ 723, Issue 1, 869-894.

[223] Pacini, F.: 1967, Nature 216, Issue 5115, 567-568.

[224] Paczynski, B.: 1986, ApJ 308, L43-L46.

[225] Padovani, P.: 2018, in Protoplanetary disks seen through the eyes of new-generation high-resolution instruments. Online at https://indico.ict.inaf.it/event/631/, jedi2018, id. 6.

[226] Padovani, P., Combes, F., Diaz Trigo, M., Ettori, S., Hatziminaoglou, E. et al.: 2017, arXiv:1705.06064.

[227] Panagia, N., Fall, S.M., Mobasher, B., Dickinson, M., Ferguson, H.C. et al.: 2005, ApJ, 633, Issue 1, L1-L4.

[228] Paredes, J.M., Persic, M.: 2013, talk at the Frascati Workshop 2009 on Multifrequency Behaviour of High Energy Cosmic Sources - VIII.

[229] Perlmutter, S., Aldering, G., Goldhaber, G., Knop, R.A., Nugent, P. et al.: 1999, ApJ 517, Issue 2, 565-586.

[230] Piran, T.: 1999, Phys. Rep. 314, Issue 6, 575-667.

[231] Piron, F.: 2016, C. R. Physique 17, 617-631.

[232] Planck Collaboration: 2014, A\&A 571, id. A11, 37 pp.

[233] Poggiani, R.: 2017, in The Golden Age of Cataclysmic Variables and Related Objects - IV, online at https://pos.sissa.it/cgi-bin/chairman/chlist.cgi?confid=315, id. 008. 
[234] Poggiani, R.: 2018, in Frontier Researc in Astrophysics - III, online at https://pos.sissa.it/cgi-bin/chairman/chlist.cgi?confid=331, id. 013.

[235] Pontzen, A., Governato, F.: 2014, https://it.wikipedia.org/wiki/Cosmologia del plasma.

[236] Prati, C. (on behalf of the LUNA collaboration): 2017, talk at the XV International Conference on Astroparticle and Underground Physics, 24-28 July, Subdury, ON, Canada.

[237] Prusti, T., de Bruijne, J.H.J., Brown, A.G.A., Vallenari, A., Babusiaux, C. et al. (Gaia Collaboration): 2016, A\&A 595, id.A1, 36 pp.

[238] Ramsay, S., Casali, M., Cirasuolo, M., Egner, S., Gray, P. et al.: 2016, Proc. SPIE 9908, id. 99081T, $12 \mathrm{pp}$.

[239] Rees, M.J.: 1988, in Origins, A.C. Fabian (ed.), Cambridge University Press, 1.

[240] Reig, P., Fabregat, J., Coe, M.J.: 1997, A\&A 322, 193-196.

[241] Renn, J., Sauer, T., Stachel, J.: 1997, Science 275, No. 5297, 184-186.

[242] Ressell, M.T., Turner, M.S.: 1990, Comm. Astrophys. 14, No. 6, 323-356.

[243] Riess, A.G., Filippenko, A.V., Challis, P., Clocchiatti, A., Diercks, A. et al.: 1998, AJ 116, Issue 3, 1009-1038.

[244] Riess, A.G., Strolger, L.-G., Tonry, J., Casertano, S., Ferguson, H.C. et al.: 2004, ApJ, 607, Issue 2, 665-687.

[245] Riess, A.G., Macri, L., Casertano, S., Lampeitl, H., Ferguson, H.C. et al.: 2011, ApJ, 730, Issue 2, article id. 119, 18 pp. - Erratum: 2011, ApJ, 732, 129.

[246] Ritter, H.: 1990, A\&AS 85, 1179-1256.

[247] Ritter, H.: 1992, in The Astronomy and Astrophysics Encyclopedia, Cambridge University Press, Cambridge, UK, p. 61.

[248] Rodriguez-Gil, P.: 2003, Ph.D. Thesis, La Laguna University, Spain.

[249] Scaringi, S.: 2015, talk at the Palermo Workshop on The Golden Age of CVs and Related Objects III.

[250] Schmidtobreick, L., Tappert, C.: 2014, in Stella Novae: Past and Future Decades, P.A. Woudt \& V.A.R.M. Ribeiro (Eds.). ASP Conf. Ser. Vol. 490, 29.

[251] Schmidtobreick, L., Tappert, C.: 2015, Acta Polytechnica CTU Proc. Vol. 2, 188.

[252] Schuecker, P.: 2005, In Rev. Mod. Astron. (Siegfried Röser Ed.), Vol. 18, 76-105.

[253] Schuecker, P., Caldwell, R.R., Böhringer, H., Collins, C.A., Guzzo, L., Weinberg, N.N.: 2003, A\&A, 402, 53-63.

[254] Schutz, B.F.: 1986, Nature 323, 310-311.

[255] Scott, D.A., Caciolli, A., Di Leva, A., Formicola, A., Aliotta, M. et al.: 2012, Phys. Rev. Lett, 109, Issue 20, id. 202501.

[256] Shahbaz, T., Bandyopadhyay, R.M., Charles, P.A., Wagner, R.M., Muhli, P., et al.: 1998, MNRAS 300, Issue 4, 1035-1040.

[257] Shandarin, S., Habib, S., Heitmann, K.: 2010, PhRv D, 81, Issue 10, id. 103006.

[258] Shakura, N.I.: 1972, Astron. Zh. 49, 921. 
[259] Shakura, N.I., Sunyaev, R.A.: 1973, A\&A 24, 337-355.

[260] Shaviv, N.J., Dar, A.: 1995, MNRAS 277, Issue 1, 287-296.

[261] Shimokawabe, T., Kawai, N., Mori, Y.A., Kudo, Y., Nakajima, H. et al.: 2009, AIPC 1133, 79-81.

[262] Smak, J.: 1984, PASP 96, 5-18.

[263] Smak, J.: 1985, in Multifrequency Behaviour of Galactic Accreting Sources, F. Giovannelli (Ed.), SIDEREA, Roma, Italy, pp. 17-36.

[264] Smith, K.: 2019, talk at LBNL, February.

[265] Soares-Santos, M., Palmese, A., Hartley, W., Annis, J., Garcia-Bellido, J et al.: 2019, ApJ 876, Issue 1, article id. L7, 15 pp.

[266] Soka Gakkai (Ed.): 1999, The writings of Nichiren Daishonin, Vol. I, p. 196.

[267] Spergel, D.N., Verde, L., Peiris, H.V., Komatsu, E., Nolta, M.R. et al.: 2003, ApJS, 148, Issue 1, 175-194.

[268] Stanev, T.: 2002, private communication.

[269] Staubert, R., Pottschmidt, K., Doroshenko, V., Wilms, J., Suchy, S. et al.: 2011, A\&A 527, id. A7, 5 pp.

[270] Steinhardt, P.J., Turok, N., Starkman, G.D.: 2008, PhT 61, Issue 1, 59.

[271] Straniero, O., Imbriani, G., Strieder, F., Bemmerer, D., Broggini, C. et al.: 2013, ApJ, 763, id. 100, $10 \mathrm{pp}$.

[272] Straniero, O., Bruno, C.G., Aliotta, M., Best, A., Boeltzig, A. et al.: 2017, A\&A 598, A128.

[273] Strieder, F., Limata, B., Formicola, A., Imbriani, G., Junker, M. et al.: 2012, PhL B, 707, Issue 1, 60-65.

[274] Sunyaev, R.A., Zeldovich, Ia.B.: 1980, ARA\&A 18, 537-560.

[275] Tang, C-H., Huang, Y-F., Geng, J-J, Zhang, Z-B.: 2019, ApJS 245, Issue 1, article id. 1, 18 pp.

[276] Tanvir, N.R., Levan, A.J., Fruchter, A.S., Hjorth, J., Hounsell, R.A. et al.: 2013, Nature, 500, 547-549.

[277] Tello, J.C., Riva, A., Hiriart, D. \& Castro-Tirado, A.J. (Eds.): 2014, III Workshop on Robotic Autonomous Observatories, RMxAC, Vol. 45.

[278] Teymourian, A.: 2004, in Proc. of the Fall 2004 Astronomy 233 Symposium on Measurements of the Hubble Constant, D.B. Campbell \& J. Deneva (Eds), offered by the Cornell University Astronomy Department and the College of Arts and Sciences under the John S. Knight Institute Sophomore Seminar Program, p. 55-61.

[279] The CMS Collaboration: 2012, Science, 338 (6114), 1569-1575.

[280] Thompson, C., Duncan, R.C., 1995, MNRAS, 275, 255-300.

[281] Thompson, C., Duncan, R.C., 1996, ApJ, 473, 322-342.

[282] Tolstoy, E., Hill, V., Tosi, M.: 2009, ARA\&A 47, Issue 1, 371-425.

[283] Tonry, J.L., Schmidt, B.P., Barris, B., Candia, P., Challis, P. et al.: 2003, ApJ, 594, 1-24. 
[284] Topchiev, N.P., Galper, A.M., Bonvicini, V., Adriani, O., Aptekar, R.L. et al.: 2015, in Proceedings of the 34th International Cosmic Ray Conference (ICRC2015), Online at http://pos.sissa.it/cgi-bin/reader/conf.cgi?confid=236, id.1026.

[285] Topchiev, N.P., Galper, A., Bonvicini, V., Arkhangelskaja, I., Arkhangelskiy, A. et al.: 2017a, in 35th International Cosmic Ray Conference, Online at https://pos.sissa.it/cgi-bin/reader/conf.cgi?confid=301, id.802.

[286] Topchiev, N.P., Galper, A.M., Bonvicini, V., Adriani, O., Arkhangelskaja, I.V. et al: 2017b, in SVHECRI 2016 - XIX International Symposium on Very High Energy Cosmic Ray Interactions, Pattison, B. (Ed.), EPJ Web of Conferences, Volume 145, id.06001.

[287] Topchiev, N.P., Galper, A.M., Arkhangelskaja, I.V., Arkhangelskiy, A.I., Bakaldin, A.V. et al.: 2019, in International Symposium on Very High Energy Cosmic Ray Interactions (ISVHECRI 2018), Pattison, B., Itow, Y., Sako, T. \& Menjo, H. (Eds.), EPJ Web of Conferences, Volume 208, id.14004.

[288] Toshikawa, J., Kashikawa, N., Ota, K., Morokuma, T., Shibuya, T. et al.: 2012, ApJ, 750, Issue 2, article id. 137, 12 pp.

[289] Treu, T., Marshall, P.J., Clowe, D.: 2012, Am. J. Phys. 80, N. 9, 753-763.

[290] Trezzi, D., Anders, M., Aliotta, M., Bellini, A., Bemmerer, D. et al. (LUNA Collaboration): 2017, Astrop. Phys. 89, 57-65.

[291] Trümper, J., Pietsch, W., Reppin, C., Voges, W., Staubert, R., Kendziorra, E.: 1978, ApJL 219, L105-L110.

[292] Tu, Z.L., Hu, J., Wang, F.Y.: 2019, MNRAS 484, Issue 3, 4337-4346.

[293] Turner, M.S., Riess, A.G.: 2002, ApJ 569, 18-22.

[294] Tyson, J.A.: 2000, Encyclopedia of Astronomy and Astrophysics, Edited by Paul Murdin, article 2144.

[295] Tyson, J.A., Kochanski, G.P., Dell' Antonio, I.P.: 1998, ApJL 498, Issue 2, L107-L110.

[296] Villada, M., Rossi, C., Polcaro, V.F., Giovannelli, F: 1999, A\&A 344, 277-281.

[297] Vink, J., Kuiper, L.: 2006, MNRAS 370, Issue 1, L14-L18.

[298] Wang, F.Y., Dai, Z.G., Liang, E.W.: 2015, New Astr. Rev. 67, 1-17.

[299] Wang, F., Fan, X., Yang, J., Wu, X-B., Yang, Q. et al.: 2017, ApJ, 839, Issue 1, article id. 27, 8 pp.

[300] Wang, F., Yang, J., Fan, X., Yue, M., Wu, X-B. et al.: 2018, ApJ 869, Issue 1, article id. L9, 6 pp.

[301] Wang, F., Yang, J., Fan, X., Wu, X-B., Yue, M. et al.: 2019a, ApJ 884, Issue 1, article id. 30, 20 pp.

[302] Wang, F., Zou, Y-C., Liu, F., Liao, B., Liu, Y. et al.: 2019b, arXiv:1902.05489.

[303] Wang, W., Han, J., Cautun, M., Li, Z., Ishigaki, M.N.: 2019, arXiv:1912.02599.

[304] Weltman, A., Bull, P., Camera, S., Kelley, K., Padmanabhan, H., Pritchard, J. et al.: 2018, arXiv:1810.02680.

[305] Wheatley, P.J., Mauche, C.W., Mattei, J.A.: 2003, MNRAS 345, Issue 1, 49-61.

[306] Williams, D.A., Balmaverde, B., Benbow, W., Bucciantini, N., Buckley, J. et al. (for the CTA Consortium): 2019, Astro2020: Decadal Survey on Astronomy and Astrophysics, APC white papers, no. 291; Bulletin of the American Astronomical Society, Vol. 51, Issue 7, id. 291. 
[307] Williams, L.L.R., Schechter, P.L.: 1997, arXiv:astro-ph/9709059, and Astron.\& Geophys. 38, Issue 5,10 .

[308] Winn, J.N., Rusin, D., Kochanek, C.S.: 2004, Nature 427, Issue 6975, 613-615.

[309] Winstein, B.: 2007, Int. J. Mod. Phy. D 16, Issue 12b, 2563-2571.

[310] Winstein, B.: 2009, in From Quantum to Cosmos: Fundamental Physics Research in Space, Turyshev, Slava G. (Ed.), Published by World Scientific Publishing Co. Pte. Ltd., ISBN 9789814261210, pp. 697-705.

[311] Wise, J.H.: 2019, arXiv:1907.06653.

[312] Wittman, D.M., Tyson, J.A., Kirkman, D., Dell'Antonio, I., Bernstein, G.: 2000, Nature 405, Issue $6783,143-148$.

[313] Wolschin, G.: 2003, in Time, Quantum and Information, L. Castell \& O. Ischebeck (Eds.), Springer, Berlin, Germany, p. 115.

[314] Wu, Xiangping: 2008, Talk at the Summer School on Cosmic Reionization at the KIAA- PKU, Beijing, China, July 1-11.

[315] Yang, J., Wang, F., Fan, X., Yue, M., Wu, X-B. et al.: 2019, AJ 157, Issue 6, article id. 236, 7 pp.

[316] Zaldarriaga, M., Spergel, D.N., Seljak, U.: 1997, ApJ 488, Issue 1, 1-13.

[317] Zaroubi, S.: 2013, in The First Galaxies, ASSL, 396, 45-104.

[318] de Zeeuw, T., Tamai, R., Liske, J.: 2014, The Messenger 158, 3-6.

[319] Zel'dovich, Ya, B. \& Guseinov, O.Kh.: 1965, Soviet Physics Doklady, Vol. 10, p.524

[320] Zhang, B.: 2013a talk at the "Multi-Messenger Transient Workshop, KIAA, China.

[321] Zhang, B.: 2013b, in Gamma-ray Bursts: 15 Years of GRB Afterglows, A.J. Castro-Tirado, J.Gorosabel and I.H. Park (Eds.), EAS Publications Series 61, 285-293.

[322] Zhao, L., Zhang, B., Gao, H., Lan, L., Lü, H., Zhang, B.: 2019, ApJ 883, Issue 1, article id. 97,22 pp.

[323] Zinnecker, H., Yorke, H.W., 2007, Annu. Rev. Astron. Astrophys., 45, 481-563.

[324] Zwicky, F.: 1939, Phys. Rev. 55, 726-743. 